\title{
Copyright
}

by

Xiaoyan SHI

2011 
The Thesis Committee for Xiaoyan Shi

Certifies that this is the approved version of the following thesis:

Permeability Estimation of Damaged Formations Near Wellbore

APPROVED BY

SUPERVISING COMMITTEE:

Supervisor:

Kenneth Gray

Maša Prodanović 


\section{Permeability Estimation of Damaged Formations Near Wellbore}

by

Xiaoyan Shi, B.E.; M.E.

\section{Thesis}

Presented to the Faculty of the Graduate School of

The University of Texas at Austin

in Partial Fulfillment

of the Requirements

for the Degree of

Master of Science in Engineering

The University of Texas at Austin

May 2011 


\section{Dedication}

To my family and friends 


\section{Acknowledgements}

I would like to offer my sincere thanks to Dr. Gray for his support and supervision during my two years of master's study. I want to thank him for providing me the opportunity to work in the "Life-of-Well: Rock, Fluid, and Stress Systems" research program, sharing his rich experience and immense knowledge of drilling and rock mechanics, and guiding me throughout the two years of study.

I am deeply grateful to Dr. Prodanović for her guidance and encouragement during my research project. I want to thank her for patient advising as I wrote thesis and her prompt answers to any of my questions.

Special gratitude is also extended to Dr. Holder and Dr. DiCarlo for their kind help and valuable input to this work.

Many thanks are also due to my office colleagues: Vladimir Rabinovich, Cagdas Arlanogln and Berkay Kocababuc for their friendship which cheered me up during hard times.

I am deeply indebted to my friends for their advice, encouragement, support and help. Without them, I could not have accomplished nearly as much.

Finally, I would like to thank my family; their endless love and support help me through any of life's challenges. 


\begin{abstract}

\section{Permeability Estimation of Damaged Formations Near Wellbore}

\author{
Xiaoyan Shi, M.S.E. \\ The University of Texas at Austin, 2010 \\ Supervisor: Kenneth Gray \\ Co-Supervisor: Maša Prodanović
}

Formation damage is a common problem in petroleum reservoirs and happens in different stages of reservoir development from drilling to production. The causes of formation damage include particle invasion, formation fines migration, chemical precipitation, and pore deformation or collapse. Formation damage adversely affects productivity of wells by reducing the permeability of near wellbore region. Furthermore, formation damage also affects well logging results. Therefore, understanding the mechanism of formation damage is vital to predict the extent and severity of formation damage and to control it.

This thesis is focused on the study of formation damage caused by external particle invasion. A simplified numerical method based on a commercial code PFC (Particle Flow Code) is proposed to simulate the particle invasion process. The fluidparticle interaction is simplified as hydrodynamic drag forces acted on particles by fluids; the particle-grain interaction is modeled as two rigid balls on contact. Furthermore, an 
pore network flow model is developed in this study to estimate permeability of damaged formations, which contain two well-separated particle sizes.

The effects of the particle size and the initial formation porosity on formation damage are studied in detail. Our study shows that big particles tend to occupy the formation face, while small particles invade deep into the formation. Moreover, particles which are smaller than pore throats (entrances) impair permeability more than those bigger than pore throats. Our study also indicates that a higher initial formation porosity results in more particle invasion and permeability impairment. It is suggested that, in order to reduce formation damage, mud particle size distributions should be carefully selected according to given formation properties.

Although our model has some limitations, it may serve as a tool to predict formation damage according to given parameters, and to understand the mechanism of formation damage from a micro-scopic point of view. 


\section{Table of Contents}

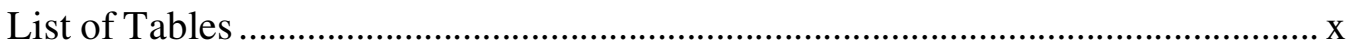

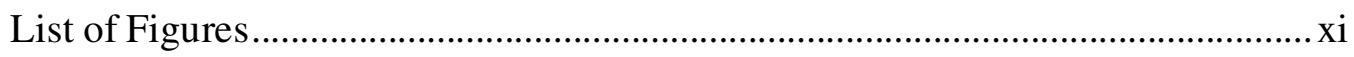

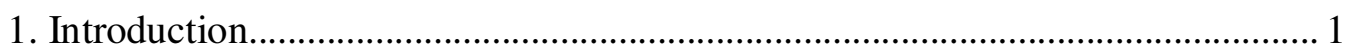

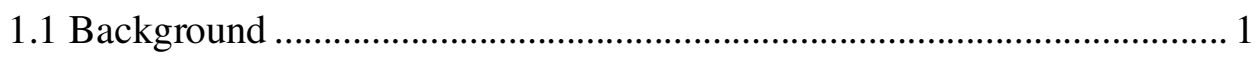

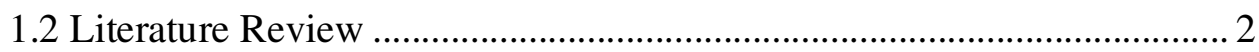

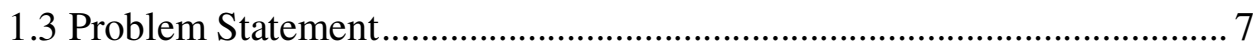

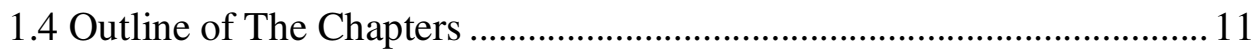

2. Discrete Grain Packing Theory and Modeling.............................................. 13

2.1 Discrete Element Method Model............................................................... 13

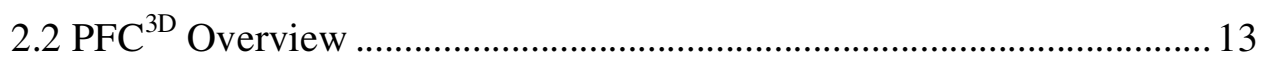

2.2.1 $\mathrm{PFC}^{3 \mathrm{D}}$ Implementation of Discrete Element Method.................. 14

2.2.2 Constitutive Contact Models ......................................................... 14

2.2.2.1 Contact-Stiffness Model ................................................. 15

2.2.2.2 Slip Model ...................................................................... 18

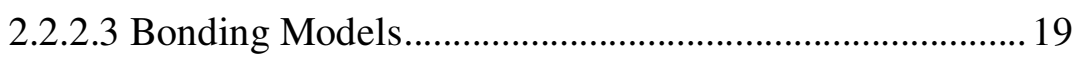

2.3 Damaged Formation Sample Modeling ................................................ 20

2.3.1 Formation Specimen Modeling ...................................................2 20

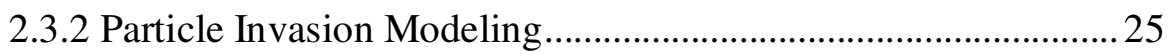

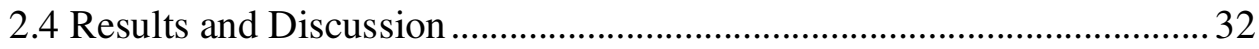

2.4.1 Effects of Drag Forces ................................................................ 32

2.4.2 Effects of Initial Formation Porosities and Particle Sizes .......... 33

2.4.2.1 Particle Invasion Results of Specimen 1 ......................... 34

2.4.2.2 Particle Invasion Results of Specimen 5 ........................ 36

2.4.2.3 Particle Invasion Results of Specimen 6 ......................... 38

2.4.2.4 Specimen with Different Particle Sizes........................... 40

2.4.2.5 Specimen with Different Formation Grain Sizes............. 41 
3. Pore Network Modeling of Granular Media......................................................... 44

3.1 Network Model Overview........................................................................ 44

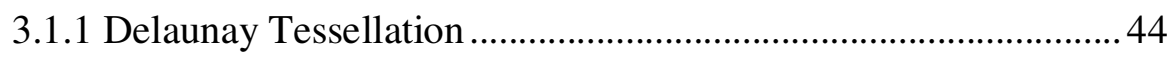

3.1.2 Pore Throat Network Based on Delaunay ................................... 45

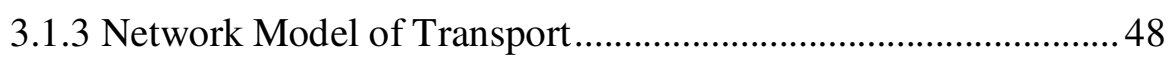

3.2 Permeability Estimation of Damaged Formations.................................. 51

3.2.1 Network Construction for Media with Two Well-Separated Grain

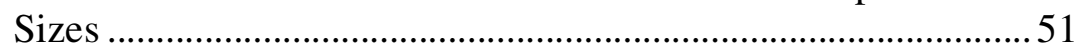

3.2.2 Pore Throat Porosity Estimation ...................................................55

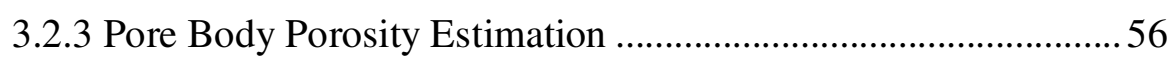

3.2.4 Pore Throat Conductance Calculation ..........................................57

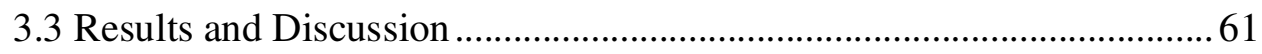

3.3.1 Permeability Impairment Study of Specimen 1........................... 61

3.3.2 Permeability Impairment Study of Specimen 5...........................69

3.3.3 Permeability Impairment Study of Specimen 7.......................... 78

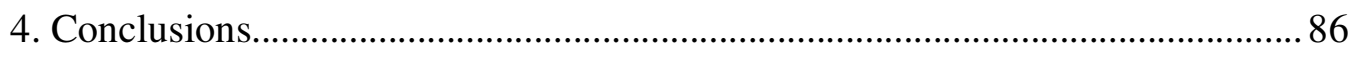

4.1 Effects of Particle Sizes on Formation Damage ...................................... 86

4.2 Effect of Initial Formation Porosity on Formation Damage ................... 90

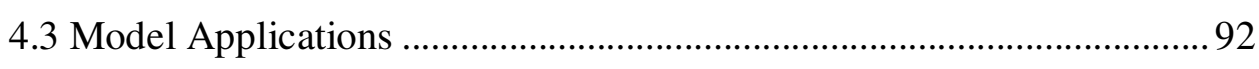

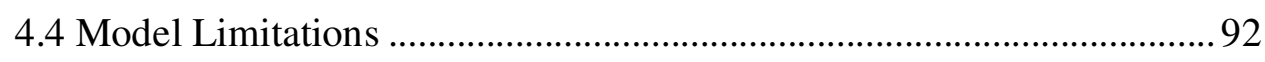

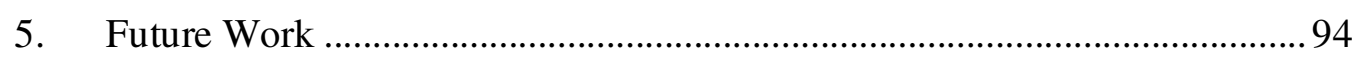

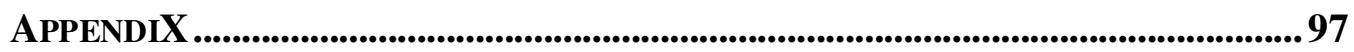

A. Generate a Dense Sphere Pack in PFC ${ }^{3 \mathrm{D}}$ ….................................................. 97

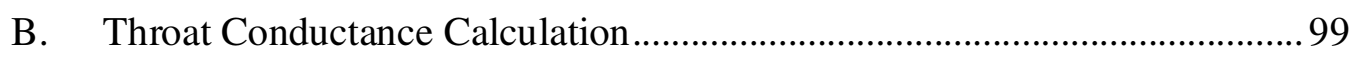

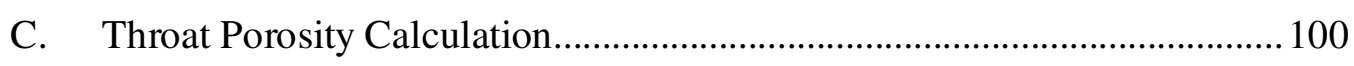

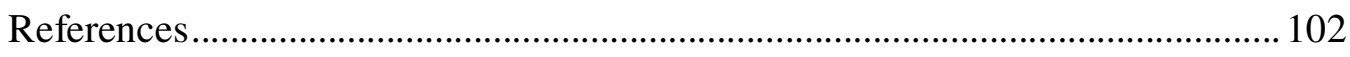




\section{List of Tables}

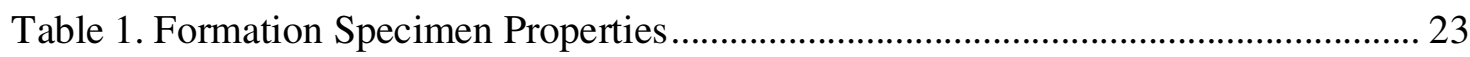

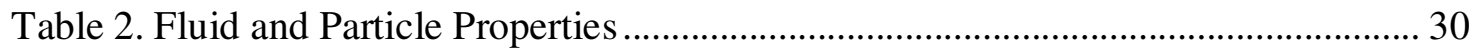

Table 3. Input Parameters and Results of the Simulation ................................................ 32

Table 4. Numerical simulation settings and results of particle invasion. The formation specimen is represented by an equal size sphere packing, and the particle size is uniform.

Table 5. Numerical simulation results of particle invasion. The formation specimen is represented by an equal size sphere packing, and the particle size is 5-10 times smaller than formation grain size.

Table 6. Numerical simulation results of particle invasion. The grain sizes of the sphere

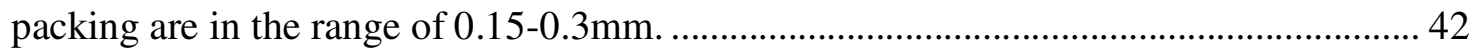

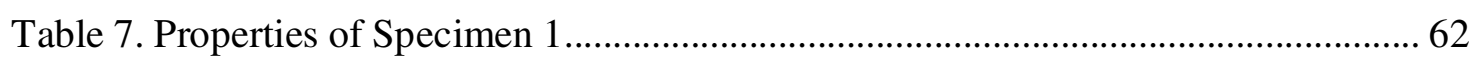

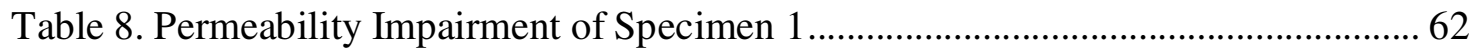

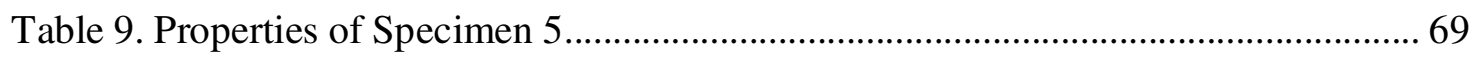

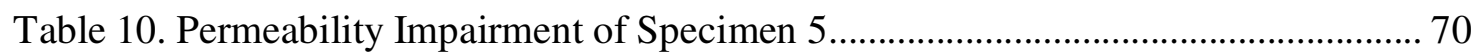

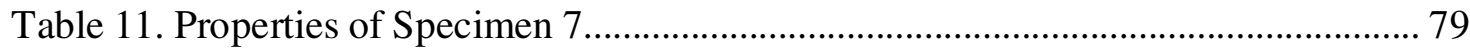




\section{List of Figures}

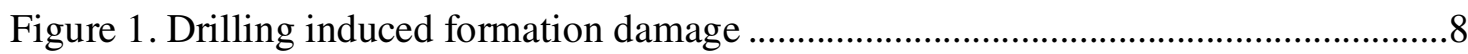

Figure 2. Dual networks of grains (in black) and pores (in red) in a porous medium ....... 10

Figure 3. Calculation cycle in PFC3D [13]. Law of motion is indeed the second Newton's Law $(\mathrm{F}=\mathrm{ma})$ which is integrated to calculate displacement of each particle in the network.

Figure 4. Contact force of two overlapping particles

Figure 5. Relationship between contact forces and relative displacements. (A) illustrates a linear relationship between the normal force and the normal overlap; the slope of the line is normal stiffness $\mathrm{Kn}$; (B) illustrates a linear relationship between the shear force and the cumulative shear displacement; the slope of the line is shear stiffness Ks. 16

Figure 6. Slip model introduces maximum shear force before two contacting particles slip one another. 18

Figure 7. Two bonding models: contact-bond model and parallel-bond model 19

Figure 8. Grain size distribution curve of natural Antler sandstone (generated according to the data from [17])

Figure 9. Formation specimen generated from PFC3D

Figure 10. Stress-strain curve of the modeled formation specimen

Figure 11. Three forces acts on a suspended particle: fluid drag force, buoyant force and gravitational force.

Figure 12. Different models of the particle-grain interaction. In the left diagram, the particle continues to travel along the fluid trajectory when it comes into contact with the formation grain. This interaction behavior was modeled in previous studies. In the right 
diagram, the movement of the particle is resulted from the contact force between the formation grain and the particle. This interaction behavior is modeled in this study....... 28 Figure 13. Simulation of particle invading into formation matrix. The big ball assembly represents formation specimen, and the small gray balls represent invading particles. (A) shows simulation setup. (B) and (C) indicate invasion results. In figure (B), formation has initial porosity as $30 \%$, and particles are three times smaller than formation grains. In figure(C), initial porosity of formation is $40 \%$, and particles are ten times smaller than

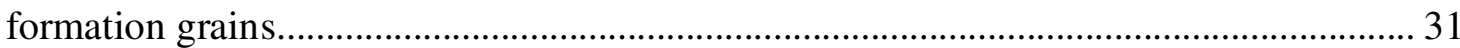

Figure 14. Normalized drag forces vs invasion depth.......................................................... 33 Figure 15. Fraction of invaded particles along the distance from the formation face. The formation specimen has initial porosity $30 \%$, and the size of invaded particles is three times smaller than that of formation grains.

Figure 16. Formation porosity reduces due to the particles invasion. The original formation porosity is about $30 \%$ and the particle size is 3 times smaller than the formation grain size 36

Figure 17. Fraction of invaded particles along the distance from the formation face. The formation specimen has initial porosity of $40 \%$, and invaded particles are five times smaller than formation grains.

Figure 18. Formation porosity reduces due to particles invasion. The original formation porosity is about $40 \%$ and the particle size is five times smaller than the formation grain size 38

Figure 19. Fraction of invaded particles along the distance from the formation face. The formation specimen has initial porosity of $40 \%$, and the size of invaded particles is ten times smaller than that of formation grains. 
Figure 20. Formation porosity reduces due to particle invasion. The original formation porosity is about $40 \%$ and the particle size is ten times smaller than the formation grain size.

Figure 21. Invaded particles in Specimen 7. Red balls represent particles with size in the range of $0.045-0.05 \mathrm{~mm}$; and the size range of black balls is $0.025-0.03 \mathrm{~mm}$.

Figure 22. Formation porosity reduces due to the particles invasion. This plot is generated from Specimen 8 42

Figure 23. 2D Delaunay tessellation 45

Figure 24. 3D Delaunay tessellation [29]. Tetrahedra vertices on the right correspond to sphere centers. 45

Figure 25. Tetrahedron from Delaunay Tessellation 46

Figure 26. Two kinds of pore throat radius which can be used in network model calculation. Inscribe radius is obtained by finding the inscribe circle; and effective radius is calculated by equivalent flow area 47

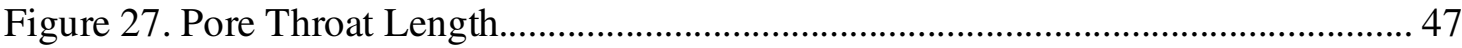

Figure 28. Setup for fluid transport simulation of the porous medium [8]....................... 48

Figure 29. Network representation of the transport simulation........................................ 49

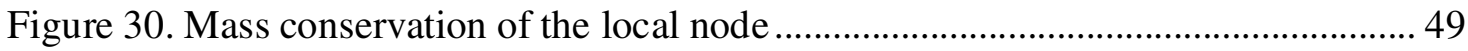

Figure 31.2D Delaunay tessellation of spheres with two well-separated sizes ................ 52

Figure 32. 3D Delaunay tessellation of spheres with two well-separated sizes ................ 52

Figure 33. In the left diagram, a large number of triangles is created from the $2 \mathrm{D}$

Delaunay tessellation of the disk pack by considering all the particles. In the right diagram, much fewer triangles are created from the 2D Delaunay tessellation of the disk pack by considering just big particles. 
Figure 34. A large number of tetrahedral is created from the 3D Delaunay tessellation of the sphere pack by considering all the particles.

Figure 35. A smaller number of tetrahedra is created from the 3D Delaunay tessellation of the sphere pack by considering just big particles 54

Figure 36. A schematic of invaded particles deposited in the pore throat...... 55 Figure 37. A particle is partially inside a pore throat. The intersection between a sphere and a cylinder is a curved surface. 56

Figure 38. Invaded particles are deposited in a pore body 57

Figure 39. A pore throat is blocked by an invaded particle .................................................58

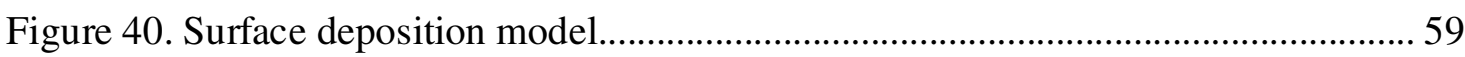

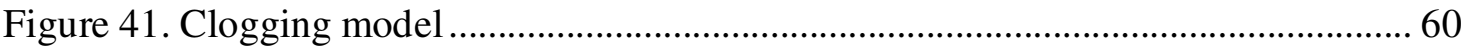

Figure 42. PDF plot of pore throat size (radius) and pore body size (radius). 63

Figure 43. Pore throat radius is the radius of a sphere inscribed among the spheres defining the throat. In the left figure, three grains have some overlap, but there is still void space among them, thus an inscribed sphere can be found. In the right figure, three grains overlapped each other heavily with, no void space left among them, and thus it is

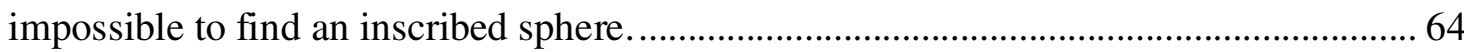

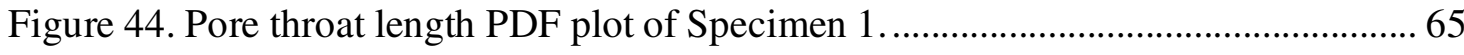
Figure 45. Histogram of all the pore throats and blocked pore throats for Specimen 1 along the distance from the formation face. 65

Figure 46. PDF plot of pore throat porosity. This plot is generated from Specimen 1..... 66 Figure 47. PDF plot of original pore volume and the pore volume with small particles deposited in the pore bodies. This plot is generated from Specimen 1

Figure 48. PDF plot of original conductance and reduced conductance. This plot is generated from Specimen 1 
Figure 49. Normalized formation permeability along the distance from the formation

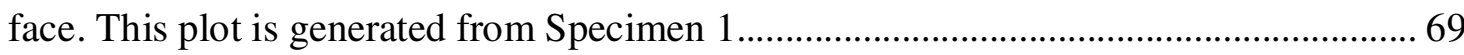
Figure 50. PDF plot of the pore throat size and the pore body size for Specimen 5........ 71 Figure 51. PDF plot of pore throat length. The plot is generated from specimen 5......... 72 Figure 52. Histogram of all the pore throats and blocked pore throats along the distance

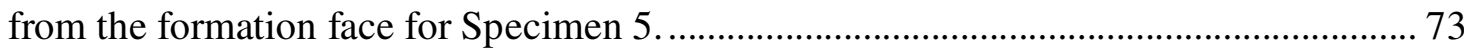

Figure 53. PDF plot of the pore throat porosity from Specimen 5................................... 74 Figure 54. PDF plot of pore volume reduction due to invaded particles for Specimen 5.74 Figure 55. PDF plot of pore throat conductance for Specimen 5..................................... 75 Figure 56. Deposition distibution of pore throats in Specimen 5 with porosity lower than $40 \%$ Figure 57. Deposition distibution of pore throats in Specimen 5 with porosity in the range of $40 \%-60 \%$. 77

Figure 58. Normalized permeability along the distance from the formation face of

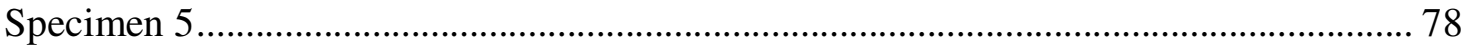

Figure 59. PDF plot of the pore throat size and the pore body size for Specimen 7 ......... 80

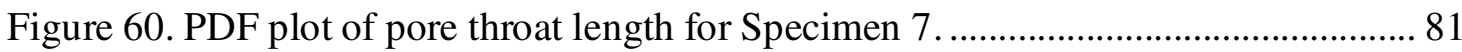

Figure 61. Histogram of all the pore throats and blocked pore throats along the distance from the formation face for Specimen 7.

Figure 62. Histogram of all the pore throats and blocked pore throats along the distance from the formation face. The plot is generated from Specimen 7.

Figure 63. PDF plot of the original pore volume and the pore volume with small particles deposited in the pore bodies for Specimen 7. 84

Figure 64. PDF plot of pore throat conductance for Specimen 7. 84 
Figure 65. Normalized permeability along the distance from the formation face of Specimen 7 85

Figure 66. Porosity reduction along the distance from the formation face for different particle sizes. The formation specimen has initial porosity as $30 \%$ 86

Figure 67. Total porosity reduction due to external particle invasion. The initial porosity of the specimen is $30 \%$. The particle sizes are 3 times smaller, 5 times smaller, 5-10 times smaller and 10 times smaller than formation grain size

Figure 68. Invaded particle fraction along the distance from the formation face. The initial porosity of the specimen is $30 \%$; and the particle sizes are 3 times smaller, 5 times smaller, 5-10 times smaller and 10 times smaller than the formation grain size. 88 Figure 69. Permeability impairment due to particle invasion along the distance from the formation face. The initial porosity of the specimen is $30 \%$; and the particle sizes are 3 times smaller, 5 times smaller, 5-10 times smaller and 10 times smaller than the formation grain size

Figure 70. Porosity reduction vs. the distance from the formation face for different initial formation porosities. The invaded particle size is ten times smaller than that of the formation grains 90

Figure 71. Invaded particle fraction along the distance from the formation face. The particle size is ten times smaller than formation grains; and the initial formation porosity is $30 \%$ and $40 \%$. 91 Figure 72. Permeability impairment due to particle invasion along the distance from the formation face. The particle size is ten times smaller than formation grains; and the initial formation porosity is $30 \%$ and $40 \%$. 91

Figure 73. A sphere pack with a smaller grain size is generated first 97 
Figure 74. The grain size of the sphere pack is increased to the target size. Computation cycle is performed to relase high internal forces caused by overlapping between particles. 


\section{Introduction}

\subsection{BACKGROUND}

Formation damage is a common problem in petroleum reservoirs, and happens in different stages of reservoir development from drilling to production. The causes of formation damage include particle invasion, formation fines migration, chemical precipitation, and pore deformation or collapse. The extent of formation damage depends on fluid properties, rock properties and fluid-rock interactions. Formation damage adversely affects production of petroleum reservoirs by reducing the permeability of the near wellbore region. A small zone of reduced permeability often greatly reduces the productivity of a petroleum reservoir. Furthermore, formation damage also affects well logging results, because most well logging tools can only measure the data within a rather shallow region that is most likely damaged. Therefore, understanding the mechanism of formation damage and the factors controlling its severity of are vital for improving the accuracy of formation evaluation and the efficiency of reservoir production. Well testing techniques provide approaches to determine formation damage near the wellbore; however, those techniques only provide the skin factor as an overall measure of the formation damage. Mathematical models combined with laboratory studies can help provide insights into the spatial development and causes of formation damage. Due to the complexity of mathematical models and the difficulty in determining the model parameters, the use of mathematical models in actual reservoir analysis and development is rather limited. 


\subsection{LITERATURE REVIEW}

There are many experimental studies on formation damage in petroleum reservoirs, but few reported mathematical models of this process. The published models can be categorized into two major groups: macroscopic and microscopic models. Macroscopic models use continuity, momentum and rate equations averaged over the representative elementary volume of porous media to describe the formation damage. Network models provide an alternative to the macroscopic approach by focusing on the fines transport and filtration process in porous media. The network models use a collection of interconnected pores and pore throats to represent the porous medium and describe the formation damage processes on a pore scale.

\section{Macroscopic Models}

Using a macroscopic model, Wojtanowicz et al. [1] studied a thin slice of a porous material. In their study, the porous medium was viewed as a bundle of hydraulic tubes linking the inlet and outlet pores of the core, and the permeability damage was assumed to be caused by three mechanisms: 1) Gradual pore blocking by surface deposition, 2) Single pore blocking by screening and 3) Pore volume filling by straining. The number of tubes blocked by a single particle was estimated as the ratio of the total volume of pore throat blocking particles to the volume of a single particle of the critical size. The critical size refers to the mean size of the critical pore constrictions in the core. The macroscopic particle mass balance calculates the permeability reduction due to pore volume filling. This is an analytic model with low computational demands, and applies when only a single mechanism dominates the formation damage process.

Khilar and Fogler [2] proposed a well-mixed compartments model. This model assumes that particles are deposited only at pore throats and that the rate of deposition is 
proportional to the flowing phase particle concentration. They also suggested the critical particle concentration above which pore throats were clogged and the particles could not travel between pore bodies. They calculated permeability reduction based on the HagenPoiseuille flow assumption of flow through the pore throat.

Gruesbeck and Collins [3] suggested a maximum gradient modeling approach. Only external particle invasion is considered in their model. They proposed a phenomenological theory based on experimental work. The main concept of the theory is to partition the porous medium at any cross section into parallel plugging and nonplugging pathways according to the particle and pore size distribution. Local laws of deposition and entrainment are studied, and their studies indicated the critical flow rate for the entrainment of particles from pore surfaces. Although the proposed model is relatively simple, it appears to be adequate for describing a broad class of filtration and entrainment phenomena.

\section{Microscopic Models}

The Sharma and Yorstsos [4] model is based on the concept of population balance of various entities over the effective medium. The porous medium is represented by a network of pore bodies and pore throats. In this model, permeability reduction is caused by two mechanisms: 1) Particles larger than pore throat block the flow; 2) Particles much smaller than the pore throats are deposited and reduce the size of the pore throat gradually. Because more and more particles plug the porous medium and dynamically change pore throat sizes, the effective-medium approximation can not accurately predict the permeability. Therefore, quantitative validation of this model against the experimental data is relatively difficult.

Rege and Fogler [5] developed a 2D network model to study and describe formation damage resulting from particle entrapment in porous media. This model uses 
the concept of flow-biased probability for the movement of particles through different flow paths. The effects of various factors, which include network size, particle size distribution and pore size distribution, on permeability reduction were studied. However, parameters used in the model are adjusted to the experimental data and the model is very computationally demanding.

During cementing the well, some cement particles might damage nearby formation. The dominant formation damage mechanism during cement operations was studied by Bortal-Nafaa and Gouvenot [6], and the main type of damage was identified as pore clogging. Authors created a sandstone specimen using Discrete Element Method (DEM) and the micro-mechanical properties of the generated sand grains were tuned to match the macro-mechanical behavior of the real sample. Furthermore, the injection process was simulated by injecting cement particles into the sandstone sample and transporting them through it. Because it is not possible to model the fluid in the DEM code, the drag force exercised by the fluid on the cement particles was considered instead. The drag force depends on the relative velocity between the fluid and the particle it is transporting, as well as on the size of the suspended element. Due to the continuously changing particle velocity, the drag forces also changed and were regularly reinitialized. The numerical simulation results were compared to the experimental results and found to fit very well.

Kim and Whittle [7] presented a numerical model for simulating pore-scale particle deposition and clogging. The invading fine particles were under the action of hydrodynamic and gravitational forces. The suspended fines were assumed to settle at the velocities according to Stokes' law and the attachment of the fines to the pore surface was calculated by the probability model. The proposed numerical analysis solves the Stokes' flow equations for an incompressible fluid using a multi-grid finite difference 
method. A large number of numerical simulations were conducted to study the process of spherical particle infiltration in a cylindrical model pore, and the results show that the particle collection efficiency is almost constant through different stages of the deposition. They also reported that particle size and settling velocity controlled the maximum height of the particle mound, which could be correlated with the differential pressure.

Li and Holt [8] proposed a fluid coupled particle model, which is a combination of a discrete particle model and a flow network model, to study stress-dependent permeability. They developed the discrete particle model based on the commercial code PFC (Particle Flow Code) to simulate the reservoir rock deformation. The numerical model developed illustrates non-linear evolution of depletion-induced compaction and stress path for a model rock (grain packing), and subsequently predicts permeability of the damaged formation. To simulate general particle shape and particle crushing during compaction, large assemblies of spherical particles (a.k.a. super-particles) are introduced to model breakable particles of general shapes. They use a network model (based on tetrahedral mesh constructed from particle centers, see section on Delaunay tessellation later) to study single phase permeability through the inter-particle pore space. As the formation deforms, the tetrahedral mesh is distorted, and the permeability estimate is updated accordingly. Although in this work we use similar tools, our specific formation damage path is different (mud invaded formation as opposed to compaction).

Macroscopic models do not realistically represent pore scale dynamics of filtration and the mounding and clogging of pores due to the infiltration. Network models, on the other hand, are means to model pore scale topology of porous media. The network framework can incorporate pore-scale models of the transport and deposition processes. However, exact interaction theory/model for a particle and pore-grain surface of general shape is not known, and thus hard to model. 


\section{Experiments}

Formation damage experiments are indispensable for phenomena observation and model validation. It is worthwhile to have a brief review of different experimental methods of formation damage.

Core flooding approach is widely used for the formation damage experiments. Van Oort et al. [9] conducted core-flow tests to verify their model results. Their experimental equipment consisted of two pressure vessels, which were filled with injection fluids and could be pressurized, and five pressure transducers connecting to the cores at certain intervals. During the experiment, initial permeability of different sections was measured first by flooding the core using pure water. Then, fluids, which contain solids with a known size distribution, were injected to determine the core impairment. The pressure drop at each interval was recorded as a function of time, and then was used to estimate the permeability of each interval. Moreover, the particle size distribution and particle concentration were measured at the inlet fluids and the outlet fluids respectively, so that the amount of particles deposited in the core could be estimated. The results of their experiments showed that more particles deposited at the beginning sections of the core, and proportionally reduced the permeability. Further, permeability decreased in time at all of the different sections along the core (as more and more particles were deposited).

Al-Abduwani et al. [10] proposed a post-mortem analysis technique, which was consisted of 2D visual analysis of core cross section and chemical analysis, to study the deposition profile along a sandstone core. The core was flooded by synthetic produced water containing hematite particles first, and then placed into the core holders equipped with microscope. 2D images were captured for different segments and at different depths. Although, theoretically, the total surface area covered by the deposition could be better 
quantified by $3 \mathrm{D}$ images, the authors used the projected $2 \mathrm{D}$ image generated from a single image sequence to quantify hematite pixels and further determine area coverage of the deposited hematite. From the image analysis, the highest surface coverage was $14 \%$ at the injection face; and the surface coverage decreased from the injection face to deeper sections of the core, which was consistent with the experimental results of van Oort et al. Compared to the image analysis, the chemical analysis provides more accurate deposition results, although it was destructive.

X-Ray Tomography (CT scanning) [11] is another valuable tool to study the damage of a core without destroying it. The basic theory of CT scanning is that a collimated X-ray beam will be attenuated after passing through some material. The amount of attenuation is directly related to the density of the material. Therefore, CT images, which are created by measuring the attenuation, can be used to investigate the material density. Gilliland et al. [12] used CT scanning technique to study various types of formation damage, which include the damage caused by mud invasion. From a slice view they created, the presence and location of the mud invasion could be easily indentified. Moreover, they showed images of mud solid invasion extent along the core without processing images to get the quantitative estimate.

\subsection{Problem Statement}

In this thesis, we mainly study drilling induced formation damage.

During well construction, drilling fluids are used to control the borehole pressure, to reduce the temperature of the drill bits and to circulate the drilled cuttings out of the boreholes. Drilling fluids need to be carefully designed so that they can have certain density and viscosity to maintain the wellbore stability and lift the cuttings. To obtain the required viscosity and density, various solid particles are added to drilling fluids. Besides 
these solids, drilling fluids also contain cuttings which are generated during the drilling process. Because of the pressure difference between the wellbore and the formation, drilling fluids flow into the permeable formation. Consequently, the suspended particles in the fluids penetrate into the formation matrix under the action of the hydrodynamic drag forces exerted by the fluids, and then are gradually deposited on the formation grain faces. As showing in Figure 1, drilling particles penetrate into the formation matrix and are deposited in the near wellbore region. The invaded particles reduce the porosity of the formation, impair the formation permeability, and thus adversely affect the productivity of the well.

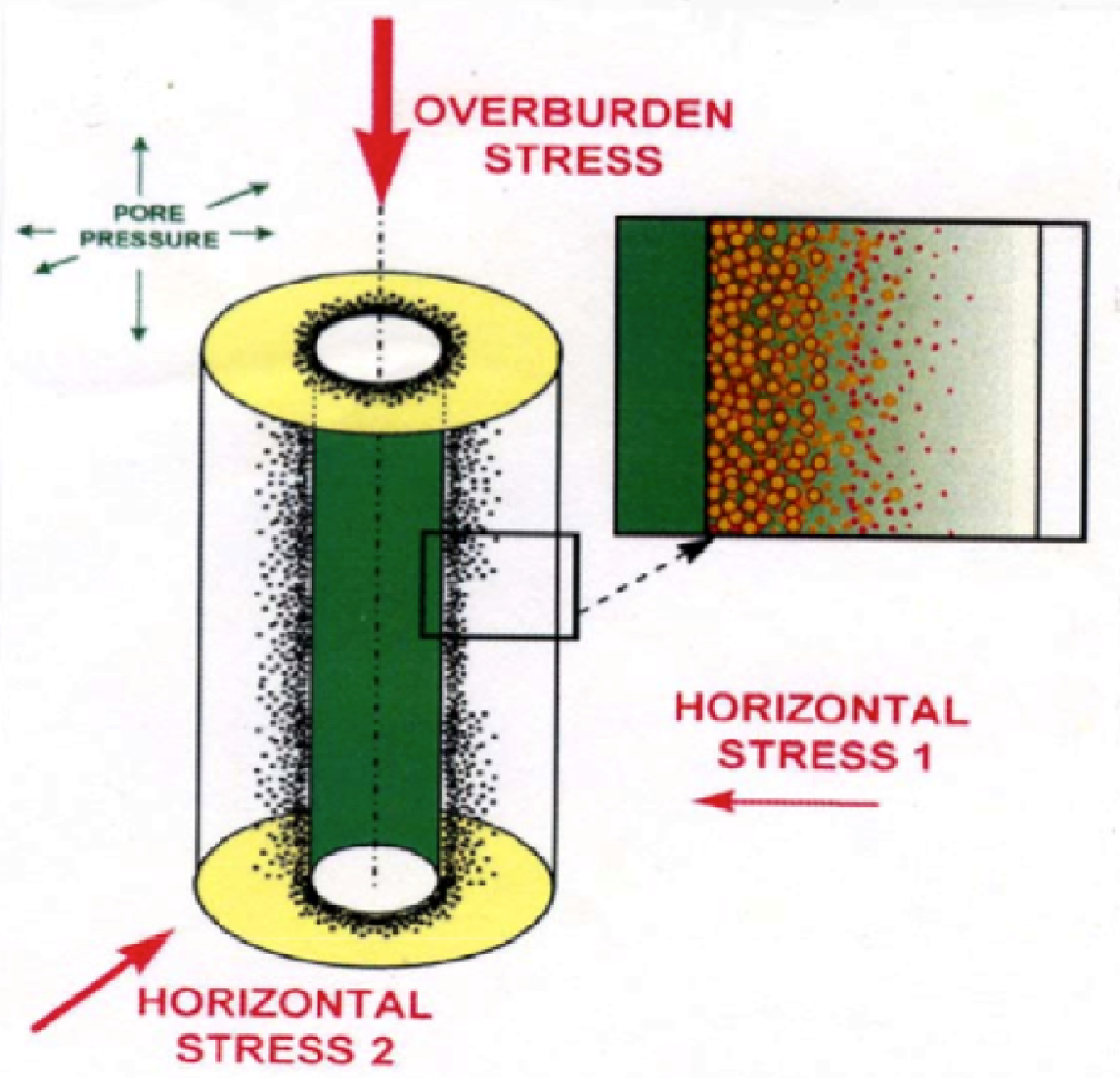

Figure 1. Drilling induced formation damage (from communication with Dr. Kenneth Gray) 
The purpose of this study is to develop a pore scale numerical model that captures pore scale details of a sample invaded by particles and fluids, and use it to estimate the permeability of the damaged formation.

The main hypotheses of our modeling are:

1. External particle invasion is the dominant mechanism of formation damage. Particles are carried into the formation by fluid and in our model they are permanently deposited. Presently we do not study dynamic dettachment and reattachment (at some different location) of particles. However, our permeability estimation approach can also be applied to the damaged formation sample regardless of how it was generated.

2. Single phase flow of an incompressible fluid is assumed.

While particle invasion also affects the relative permeability in multiphase fluid flow, we presently focus on estimating the absolute permeability of the damaged formation.

The developed model couples the geo-mechanical behavior of the formation grains with the fluid flow within the porous medium. The system in this study consists of dual networks: the network formed by the sand grains and the network of fluid flow path (see Figure 2). 


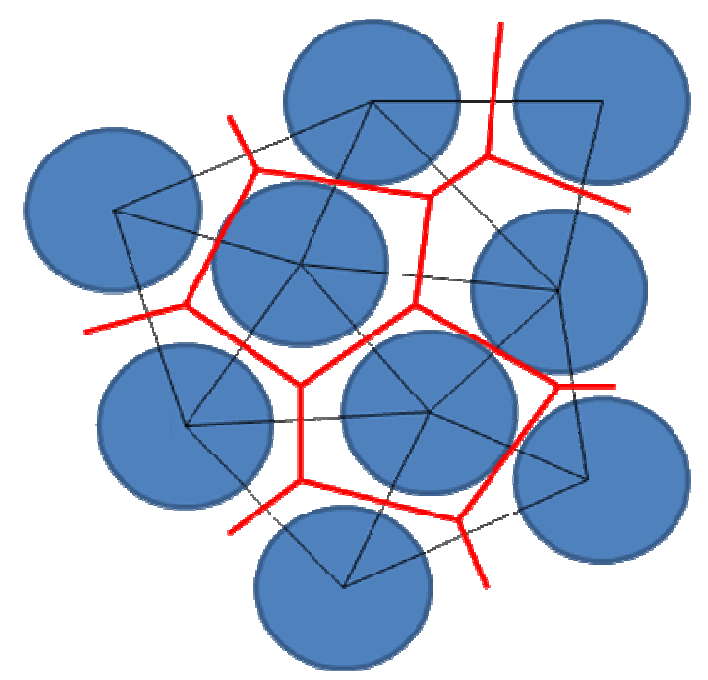

Figure 2. Dual networks of grains (in black) and pores (in red) in a porous medium

Two main advancements of this study are to generate specimens of the damaged formations and to estimate their permeability.

The damaged formation sample contains grains of two well-separated length scales, but only the larger ones are load-bearing. The specimen of the porous medium is modeled in Itasca's PFC ${ }^{3 \mathrm{D}}$ software in two steps. First, a spherical pack is generated to represent the formation sample and the micro-mechanical properties of the particles are selected to match the macro-mechanical behavior of the real sample. Then, the particle invasion process is simulated by assigning drag force and gravity force to invading particles and allowing them to transport and deposit into the porous medium. The interactions between the invading particles and the porous medium during the transport and deposition process are taken into account. The constructed porous medium from this model is mechanically stable.

A specifically tailored network model is used to estimate the permeability of the damaged formation. To obtain a physically meaningful fluid path, only big particles are included in the network construction. The deposited small particles are taken into account in estimating pore throat conductance. In this thesis, two models of estimating the pore 
throat conductance are proposed: a surface deposition model and a clogging model. The surface deposition model assumes that invading particles are deposited on the surface of grains and that the pore throat volume reduces gradually from the surface to the center. In the surface deposition model, we estimate the effective radius of the pore throat to calculate the conductance. The clogging model assumes that the invading particles clog the pore throat, thus the Carman-Kozeny model is used to estimate the permeability of the pore throat based on porosity.

The extent and severity of the formation damage depends on various factors, among which are the initial porosity and permeability of the formation and the invaded particle size. Therefore, different initial porosity and invaded particle sizes are used to estimate the permeability and analyze the effects of the two factors on the permeability damage. Furthermore, the damaged formation is categorized according to its distance from the invading start point. Different groups have different levels of permeability reduction; the ones closer to the wellbore are more severely damaged.

The permeability estimation model proposed in this thesis provides a way to understand the process of formation damage due to external particle invasion and its effects on permeability damage. The network creation and fluid model in this study has been adapted to work with two scales, which is the main novelty of this work and can be applied in other fields with similar grain size distribution.

\subsection{OUtLine OF The Chapters}

The remainder of this thesis is organized as follows:

Chapter 2 gives an overview of the discrete element method (DEM) and PFC ${ }^{3 \mathrm{D}}$ and generates the damaged formation specimens using $\mathrm{PFC}^{3 \mathrm{D}}$. 
Chapter 3 introduces the theory behind the network model and shows how the adapted model estimates the permeability of the damaged formation.

Chapter 4 presents and discusses the results of the permeability estimation model. Chapter 5 suggests future work. 


\section{Discrete Grain Packing Theory and Modeling}

\subsection{Discrete Element Method Model}

The Discrete Element Method (DEM), which was introduced by Cundall and Strack [13] to rock mechanics, models the motion of a large number of micrometer-scale size particles. In DEM, the interaction of the particles is considered as a dynamic process with states of equilibrium developing whenever the internal forces balance [14]. The dynamic process is modeled by a time-stepping algorithm, in which accelerations are assumed constant within each time step. Therefore, the movements of the individual particles can be traced. The contact forces and displacements of stressed particles are found from the movements of the particles. A force-displacement law at the contacts is applied to calculate the contact forces between two particles, and Newton's second law is used to determine the motion of each particle caused by the contact force or other forces applied on the particle.

DEM is widely applied in the rock mechanics field. Li and Holt [8] used a commercial DEM code, Itasca's Particle Flow Code (PFC), to develop a discrete particle model, which was used to study reservoir geo-mechanics. In this thesis, Particle Flow Code 3 Dimension $\left(\mathrm{PFC}^{3 \mathrm{D}}\right)$ is used to simulate the interactions between formation grains and invading particles. We start with a brief review of the $\mathrm{PFC}^{3 \mathrm{D}}$, s basic modeling capabilities.

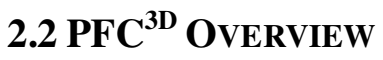

$\mathrm{PFC}^{3 \mathrm{D}}$ models a granular medium as a network of rigid spherical particles in contact. Soft contact approach, which allows rigid particles to overlap one another at contact points, is used to characterize the nature of contacts. The mechanical behavior of 
such a system is described in terms of the movement of each particle and the interparticle forces acting at each contact point.

\subsubsection{PFC $^{3 \mathrm{D}}$ Implementation of Discrete Element Method}

The calculation method in $\mathrm{PFC}^{3 \mathrm{D}}$ is a time stepping scheme [15]. In each calculation cycle, the force-displacement law is applied to each contact and the law of motion is applied to each particle, as illustrated in the Figure 3. Contacts between particles are formed or broken during the simulation. At the beginning of each time step, contacts are updated according to the relative positions between particles and contact force is calculated for each contact; then the velocity of each particle is calculated based on the resultant force acting on the particle and the new position of each particle is obtained. Besides contact force, other external forces, which are assigned directly to particles, also contribute to movement of particles.

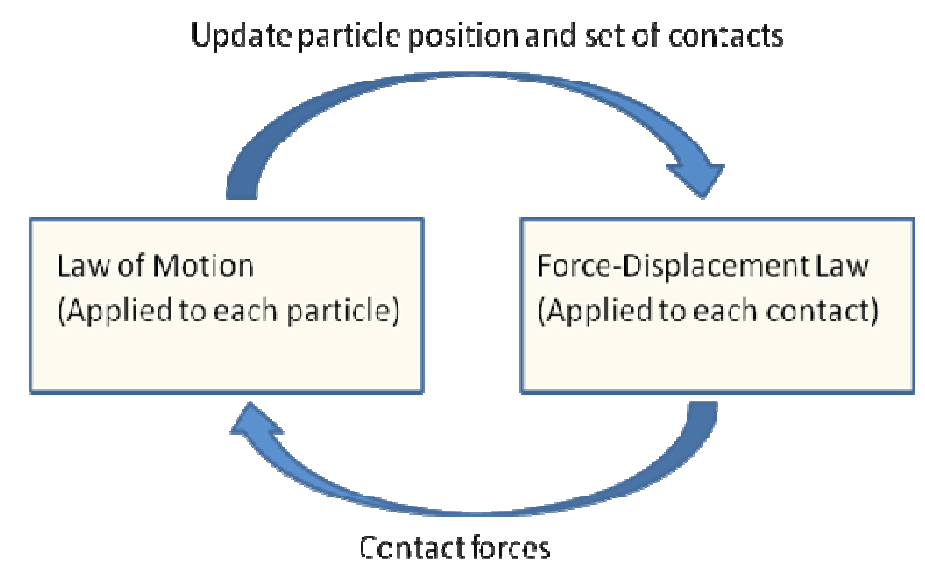

Figure 3. Calculation cycle in $\mathrm{PFC}^{3 \mathrm{D}}$ [13]. Law of motion is indeed the second Newton's Law $(F=m a)$ which is integrated to calculate displacement of each particle in the network.

\subsubsection{Constitutive Contact Models}

$\mathrm{PFC}^{3 \mathrm{D}}$ associates a simple constitutive model with each contact to simulate the overall constitutive behavior of a material. The contact constitutive model consists of three parts: a stiffness model; a slip model; and a bonding model. The contact stiffness 
model establishes an elastic relation between the contact force and relative displacement. The slip model provides a relationship between shear and normal contact forces to determine whether the two contacting balls will slip relative to one another. By enforcing bond-strength limits, the bonding model limits the total normal and shear forces required to break the contact.

\subsubsection{Contact-Stiffness Model}

The contact model in $\mathrm{PFC}^{3 \mathrm{D}}$ describes the interaction between two contacting particles or a particle and a wall which come into contact. The model establishes fundamental relationship between contact forces and relative displacement in both normal and shear directions.

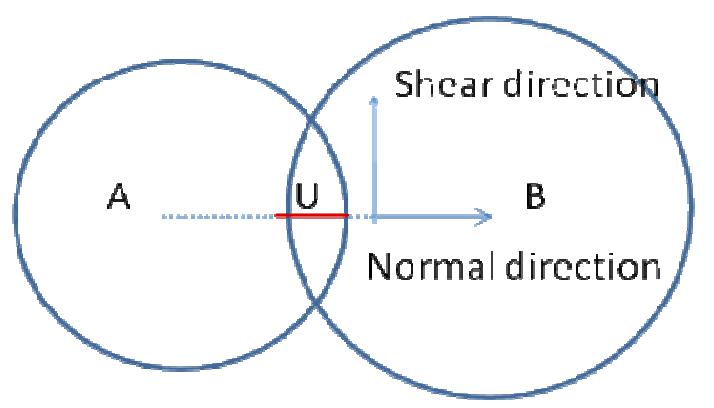

Figure 4. Contact force of two overlapping particles

In Figure 4, particle A and particle B overlaps one another. The overlap distance is $\mathrm{U}$, which can be calculated from the geometry. Assuming the radius of particle $\mathrm{A}$ is $\mathrm{R}^{\mathrm{A}}$, the radius of particle $B$ is $R^{B}$, and the distance between the centers of the two particles is $\mathrm{d}$, the overlapping distance $\mathrm{U}$ can be calculated by the following equation:

$$
\mathrm{U}=\mathrm{R}^{\mathrm{A}}+\mathrm{R}^{\mathrm{B}}-\mathrm{d}
$$

The normal direction in this local coordinate system is defined as the direction of the connection between two particle centers, and shear direction is perpendicular to the 
normal direction. The normal stiffness $\left(K_{n}\right)$ relates the total normal force $\left(F_{n}\right)$ and total displacement in normal direction $\left(\mathrm{U}_{\mathrm{n}}\right)$ via the following equation:

$$
\mathrm{F}_{\mathrm{n}}=\mathrm{K}_{\mathrm{n}} \mathrm{U}_{\mathrm{n}}
$$

The shear stiffness $\left(\mathrm{K}_{\mathrm{s}}\right)$ relates the increment of shear force $\left(\Delta \mathrm{F}_{\mathrm{s}}\right)$ to the increment of shear displacement $\left(\Delta U_{s}\right)$ via the following equation:

$$
\Delta \mathrm{F}_{\mathrm{s}}=\mathrm{K}_{\mathrm{s}} \Delta \mathrm{U}_{\mathrm{s}}
$$

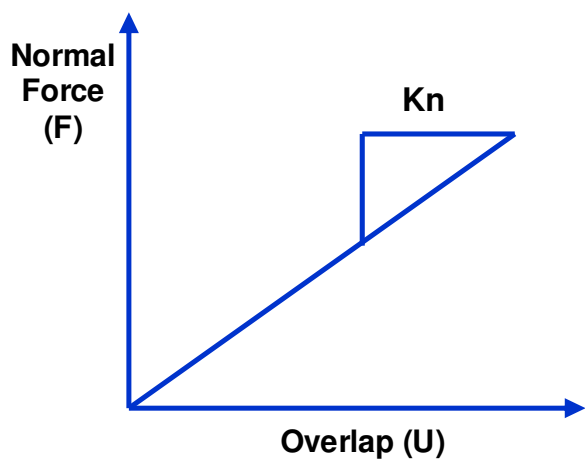

(A)

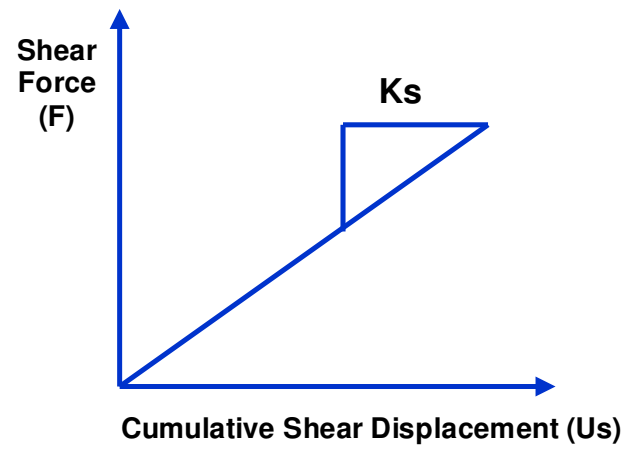

(B)

Figure 5. Relationship between contact forces and relative displacements. (A) illustrates a linear relationship between the normal force and the normal overlap; the slope of the line is normal stiffness Kn; (B) illustrates a linear relationship between the shear force and the cumulative shear displacement; the slope of the line is shear stiffness Ks.

PFC $^{3 \mathrm{D}}$ provides two models to decide the normal stiffness and shear stiffness: a liner model and a simplified Hertz-Mindlin model, which are discussed briefly below.

\section{The linear contact model}

In the linear model, the interaction between two contact particles is an analogue of the interaction of two springs. The resultant normal and shear stiffness of the contact can be calculated from each particle's normal and shear stiffness, respectively.

The resultant normal stiffness $\mathrm{K}_{\mathrm{RN}}$ of the contact can be calculated from the normal stiffness of the two particles: $K_{n}^{A}, K_{n}^{B}$ via the equation: 


$$
\mathrm{K}_{\mathrm{RN}}=\frac{\mathrm{K}_{\mathrm{n}}^{\mathrm{A}} \mathrm{K}_{\mathrm{n}}^{\mathrm{B}}}{\mathrm{K}_{\mathrm{n}}^{\mathrm{A}}+\mathrm{K}_{\mathrm{n}}^{\mathrm{B}}}
$$

Similarly, the resultant shear stiffness of the contact is calculated from the shear stiffness of the two particles: $\mathrm{K}_{\mathrm{S}}^{\mathrm{A}}, \mathrm{K}_{\mathrm{S}}^{\mathrm{B}}$ via the equation:

$$
\mathrm{K}_{\mathrm{RS}}=\frac{\mathrm{K}_{\mathrm{s}}^{\mathrm{A}} \mathrm{K}_{\mathrm{s}}^{\mathrm{B}}}{\mathrm{K}_{\mathrm{s}}^{\mathrm{A}}+\mathrm{K}_{\mathrm{s}}^{\mathrm{B}}}
$$

The accumulated displacement in shear direction cannot be directly calculated from the geometry. So the relative movement in the shear direction is recorded for each time step, which is used to do the shear displacement calculation. In our study, the linear contact model is used.

\section{The Hertz-Mindlin contact model}

PFC $^{3 \mathrm{D}}$ provides simplified Hertz-Mindlin contact model, which is a nonlinear contact model based on the theory of Mindlin and Deresiewicz [16]. The model predicts normal stiffness and shear stiffness of contact from geometry and elastic properties of two contacting particles. The elastic properties of contacting particles needed for this model are: shear modulus G and Poisson's ratio v . For particle-to-particle interaction, elastic properties of contacts are estimated as the mean values of the two particles' properties.

The contact normal secant stiffness is calculated by the equation:

$$
\mathrm{K}_{\mathrm{RN}}=\frac{2 \mathrm{G} \sqrt{2 \mathrm{R}}}{3(1-\mathrm{v})} \sqrt{\mathrm{U}}
$$

The contact shear tangent stiffness is given by the equation:

$$
\mathrm{K}_{\mathrm{s}}=\left(\frac{2\left(\mathrm{G}^{2} 3(1-\mathrm{v}) \mathrm{R}\right)^{1 / 3}}{2-\mathrm{v}}\right)\left|\mathrm{F}^{\mathrm{n}}\right|^{1 / 3}
$$

Where $\mathrm{G}$ is mean modulus of the two contacting particles;

$\mathrm{v}$ is mean Poisson's ratio of the two contacting particles;

$\mathrm{U}$ is normal displacement at the contact; 
$\mathrm{F}^{\mathrm{II}}$ is normal contact force;

$\mathrm{R}$ is calculated from the radii of the two contacting particles by equation:

$$
R=\frac{2 R^{A} R^{B}}{R^{A}+R^{B}}
$$

\subsubsection{Slip Model}

The slip model in $\mathrm{PFC}^{3 \mathrm{D}}$ describes the constitutive behavior of the particle contact occurring at a point. It allows slip by limiting the maximum shear force at the contact, which is defined by frictional resistance. Each particle has an intrinsic propertyfrictional coefficient $\mu$, and the minimum friction coefficient of the two contacting particles is used to calculate maximum shear force as showing in the following equation:

$$
\mathrm{F}_{\max }^{\mathrm{s}}=\mu\left|\mathrm{F}_{\mathrm{i}}^{\mathrm{n}}\right|
$$

where $\mathrm{F}^{\mathrm{n}}$ is the normal force at the contact.

Therefore, at each time step, the calculated shear force at the contact is compared to the maximum shear force, if it exceeds the maximum value, then slip occurs. Considering the slip model, the original relationship between shear force and cumulative shear displacement is adjusted as shown in Figure 6.

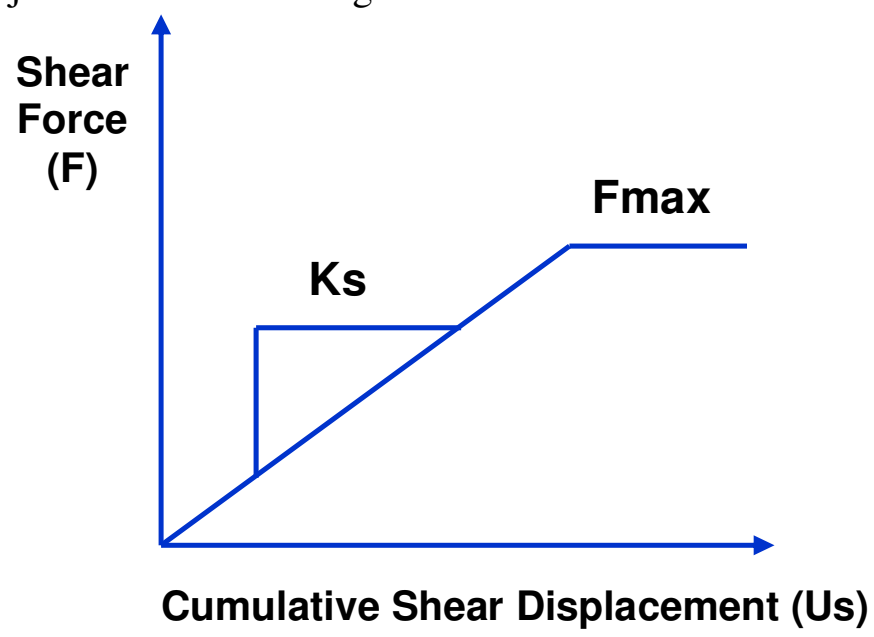

Figure 6. Slip model introduces maximum shear force before two contacting particles slip one another. 


\subsubsection{Bonding Models}

Two bonding models, a contact-bond model and a parallel-bond model, are provided in $\mathrm{PFC}^{3 \mathrm{D}}$ to bond particles together at contacts. The contact bond acts like glue joining two particles at the contact point as showing in Figure 7-A. The parallel bond is more like cementation acting over a circular cross section lying between the particles as showing in Figure 7-B.

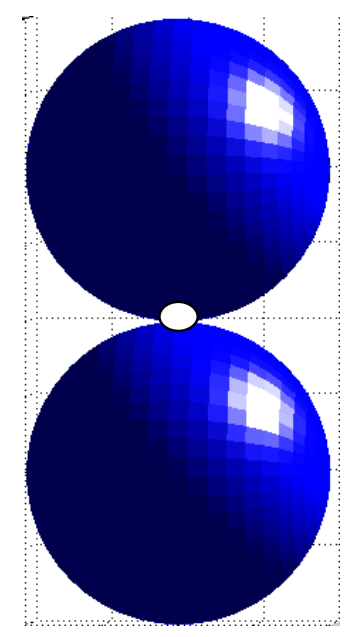

(A)

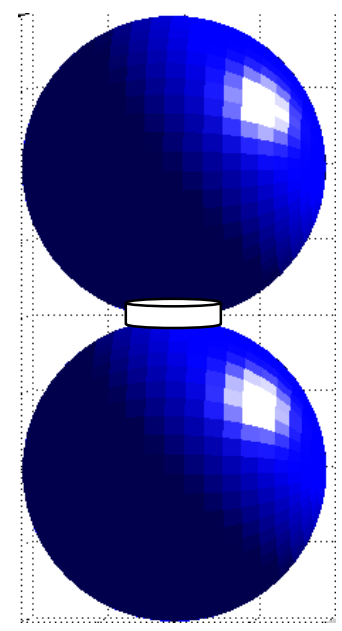

(B)

Figure 7. Two bonding models: contact-bond model and parallel-bond model.

(A) Contact bond acts on the contact point of two particles to join the two particles together; (B) Parallel bond acts on the circular cross section lying between the two particles to join them together.

The contact-bond model can be viewed as installing elastic springs in both normal and shear direction when two particles come into contact. The spring in normal direction binds two balls together by allowing tensile forces to develop at a contact, which happens when the two balls move apart from one another. The magnitude of the normal contact forces is limited by the normal contact bond strength; and the shear contact force magnitude is limited by the shear contact bond strength. 
Alternatively, the parallel bond models constitutive behavior of cementation deposited between two balls. It acts like a set of elastic springs uniformly distributed over the cementation between the two balls. These springs are parallel to the normal and shear springs of the contact model. After installation of the parallel bond, additional compression of the contact causes resistance from both cementation and two balls; on other hand, tensile force is developed by the cementation when two balls move apart. Either maximum normal stress or maximum shear stress within the cementation material exceeds in the bond strength, the parallel bond breaks.

\subsection{DAMAGED FORMATION SAMPLE MODELING}

In this thesis, it is assumed that invasion and deposition of external particles, which happens in both drilling and production stages, are the dominant factors of the formation damage. Drilling induced particle invasion is studied in this thesis, and the developed model can also be applied to production induced particle invasion. The invasion of external particles is modeled by $\mathrm{PFC}^{3 \mathrm{D}}$ in two steps: 1) formation specimen modeling; 2) simulation of particle invasion.

\subsubsection{Formation Specimen Modeling}

The invasion model in this study is aimed for poorly consolidated or unconsolidated formation. The Antler sandstone is selected as the formation to be modeled. It is weakly consolidated sandstone, which outcrops near Ardmore, Oklahoma. Wang et al. [17] did comprehensive analysis of natural Antler sandstone and its mechanical properties are available from their study. Gil et al [18] developed a numerical model based on DEM to represent the mechanical behaviors of the Antler sandstone. Similar approach is adopted in this study. We use $\mathrm{PFC}^{3 \mathrm{D}}$ to model the formation and its mechanical behavior. 
$\mathrm{PFC}^{3 \mathrm{D}}$ models formation as densely packed spherical balls. Micro-properties, such as density, friction coefficient, normal stiffness and shear stiffness, are assigned to each ball. Choosing proper micro-mechanical properties to match general behavior of macroscopic mechanical properties of formation is the key factor of successful modeling of the formation specimen. In addition to micro-mechanical properties assignment, proper selection of the grain size distribution is also important.

\section{Grain size selection}

The grain size distribution for the natural Antler sandstone was reported by Wang et al. and plotted in Figure 8 .

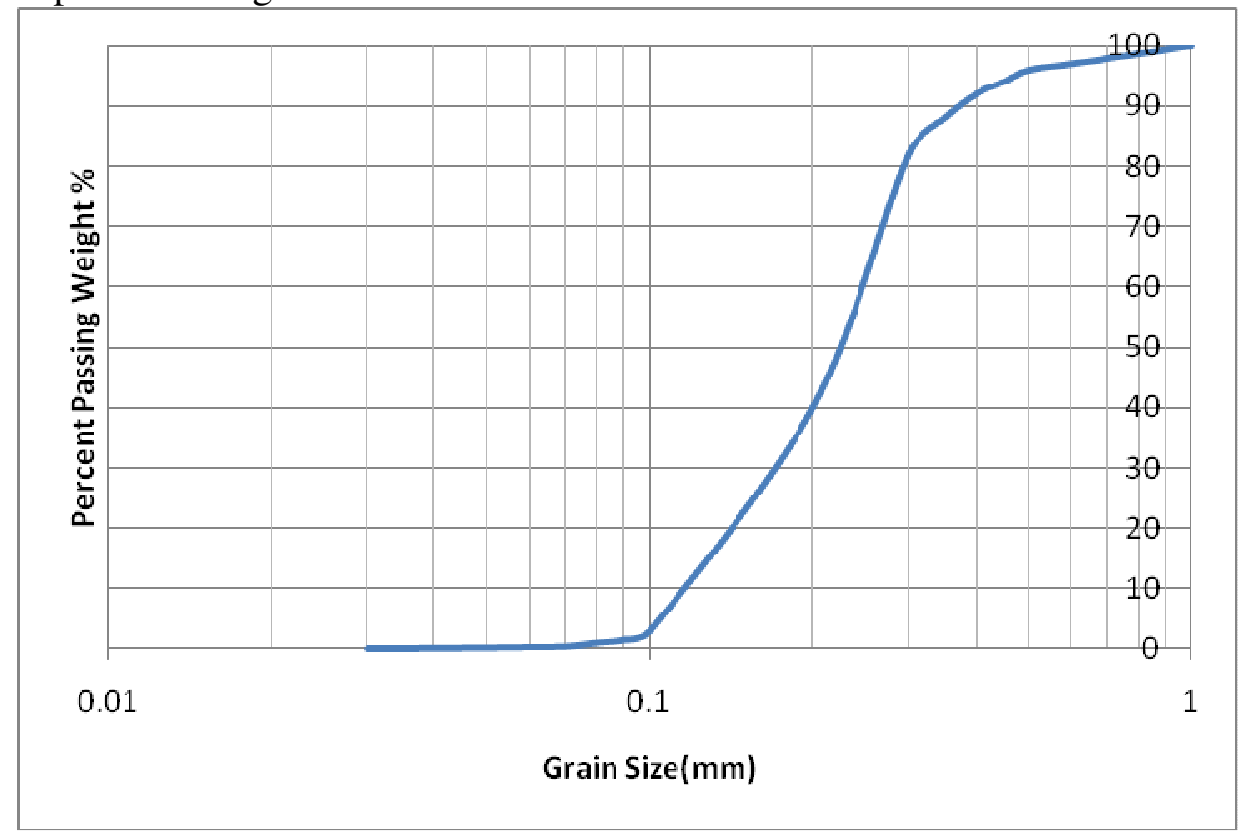

Figure 8. Grain size distribution curve of natural Antler sandstone (generated according to the data from [17])

Although the grain size distributes from 0.03 to $1 \mathrm{~mm}$, about $80 \%$ grains have the size in the range of $0.1-0.3 \mathrm{~mm}$ and the mean grain size is about $0.3 \mathrm{~mm}$. Wider grain size range rapidly slows down the simulation speeds and also causes numerical instability of 
the further network model; therefore, in this study, equal grain size $(0.25 \mathrm{~mm})$ is used for the numerical model of the Antler sands.

\section{Micro-mechanical properties calibration}

Wang et al. laboratory results show that when confining pressure increased from 6.9 to $34.5 \mathrm{MPa}$, the Young's modulus of natural Antler sandstone ranged from 4.5 to 20.7GPa; the Poisson's ratio changed from 0.2-0.3; and the peak deviatoric stress varies between $33.1 \mathrm{MPa}$ to $96.5 \mathrm{MPa}$. Micro-mechanical properties of $\mathrm{PFC}^{3 \mathrm{D}}$ grains are carefully selected and tuned to match to macro-mechanical properties of the real sample. Although some macro-mechanical properties, such as Young's modulus and Poisson's ratio, do not affect the results of the particle invasion simulation, this modeling approach can serve as the base for the future studies on other types of formation damage, for example, formation damage caused by pore collapse or sand crushing.

Quartz is the main mineral component of sand grains. According to the laboratory test results published by Gil et al., the quartz's Young's modulus is about $94.3 \mathrm{GPa}$ and the shear modulus is $34.0 \mathrm{GPa}$. Thus, these values are assigned as normal stiffness and shear stiffness to $\mathrm{PFC}^{3 \mathrm{D}}$ grains. In addition to the grain properties' selection, the selection of the bonding type and the bonding stiffness values is also important to match the macroscopic mechanical behaviors of the modeling material, because the initial value of Young's modulus of the modeled formation is directly related to the values of contact stiffness. Parallel bond model is selected for the specimen modeling, and the value of normal and shear stiffness of bonds are carefully picked and validated. We performed numerical tri-axial test against the generated sample and obtained the desired elastic properties of the sample by tuning the micro-properties. The tri-axial routine is provided by $\mathrm{PFC}^{3 \mathrm{D}}$. 
Table 1 shows the final selection of micro-properties for PFC $^{3 \mathrm{D}}$ grains. Given the confining pressure of $6.9 \mathrm{MPa}$, the resultant Young's modulus of the generated specimen is about $4.8 \mathrm{GPa}$, which matches the test results of real sample well. Moreover, the selected values of normal and shear stiffness of $\mathrm{PFC}^{3 \mathrm{D}}$ grains match the real sample properties as well. High porosity is used in the model, since weakly-consolidated sandstone tends to have more porosity.

Table 1. Formation Specimen Properties

\begin{tabular}{|c|c|c|}
\hline \\
\hline & Input Parameters & Value \\
\hline \multirow{4}{*}{$\begin{array}{c}\text { General Properties of the } \\
\text { specimen }\end{array}$} & Specimen Size & $8 * 4 * 4$ \\
\hline & Length $(\mathrm{mm}) *$ Width $(\mathrm{mm}) *$ Height $(\mathrm{mm})$ & \\
\hline & Grain Radius (mm) & 0.25 \\
\hline & Porosity & 0.3 \\
\hline \multirow{6}{*}{$\begin{array}{c}\text { Micro-Properties of PFC3D } \\
\text { grains }\end{array}$} & Grain Density $(\mathrm{kg} / \mathrm{m} 3)$ & 2650 \\
\hline & Grain Normal Stiffness (GPa) & 94.3 \\
\hline & Grain Shear Stiffness (GPa) & 34.0 \\
\hline & Friction Coefficient & 0.55 \\
\hline & Parallel Bond Normal Stiffness $(\mathrm{GPa} / \mathrm{m})$ & 95 \\
\hline & Parallel Bond Shear Stiffness $(\mathrm{GPa} / \mathrm{m})$ & 40 \\
\hline $\begin{array}{l}\text { Macro-mechanical } \\
\text { properties of the specimen }\end{array}$ & $\begin{array}{c}\text { Young's Modulus (GPa) } \\
\text { (Confining stress is 6.9 MPa) }\end{array}$ & 4.8 \\
\hline
\end{tabular}




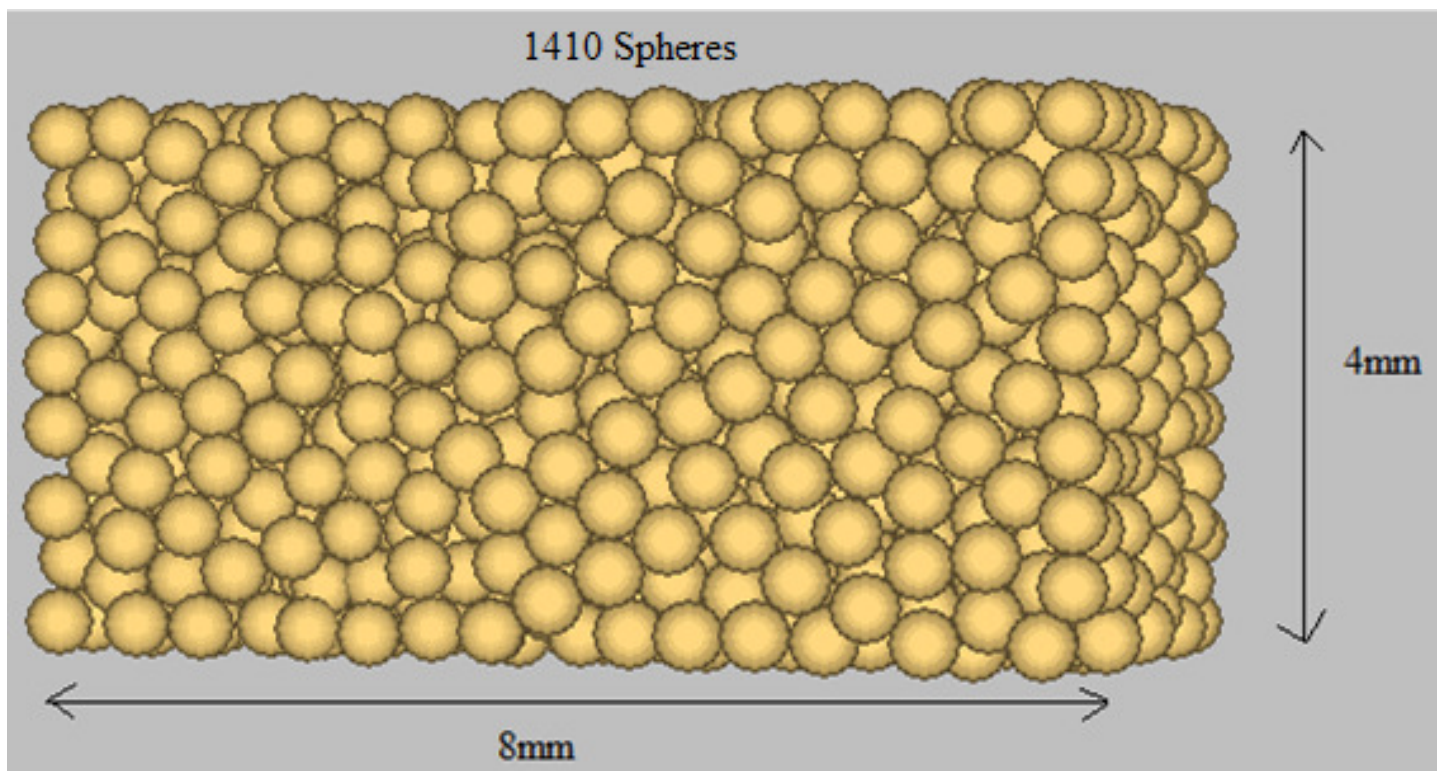

Figure 9. Formation specimen generated from $\mathrm{PFC}^{3 \mathrm{D}}$

Figure 9 shows the generated formation specimen, which includes 1410 spheres. The generated specimen is $8 \mathrm{~mm}$ long, $4 \mathrm{~mm}$ wide and $4 \mathrm{~mm}$ thick.

Figure 10 shows the stress-strain curve from the tri-axial test of the modeled formation specimen. From the curve, we can see that the Young's modulus of the generated sample is about $4.8 \mathrm{GPa}$.

Note that properties related to the bond models affect the elastic behavior of the generated sample, while they do not have effects on particle invasion; thus for the particle invasion simulation, bond properties are not set for the formation grains. The calibration process of the bond properties can be used for the future studies on fracture related formation damage or sand crushing related formation damage, as mentioned in the previous section. 


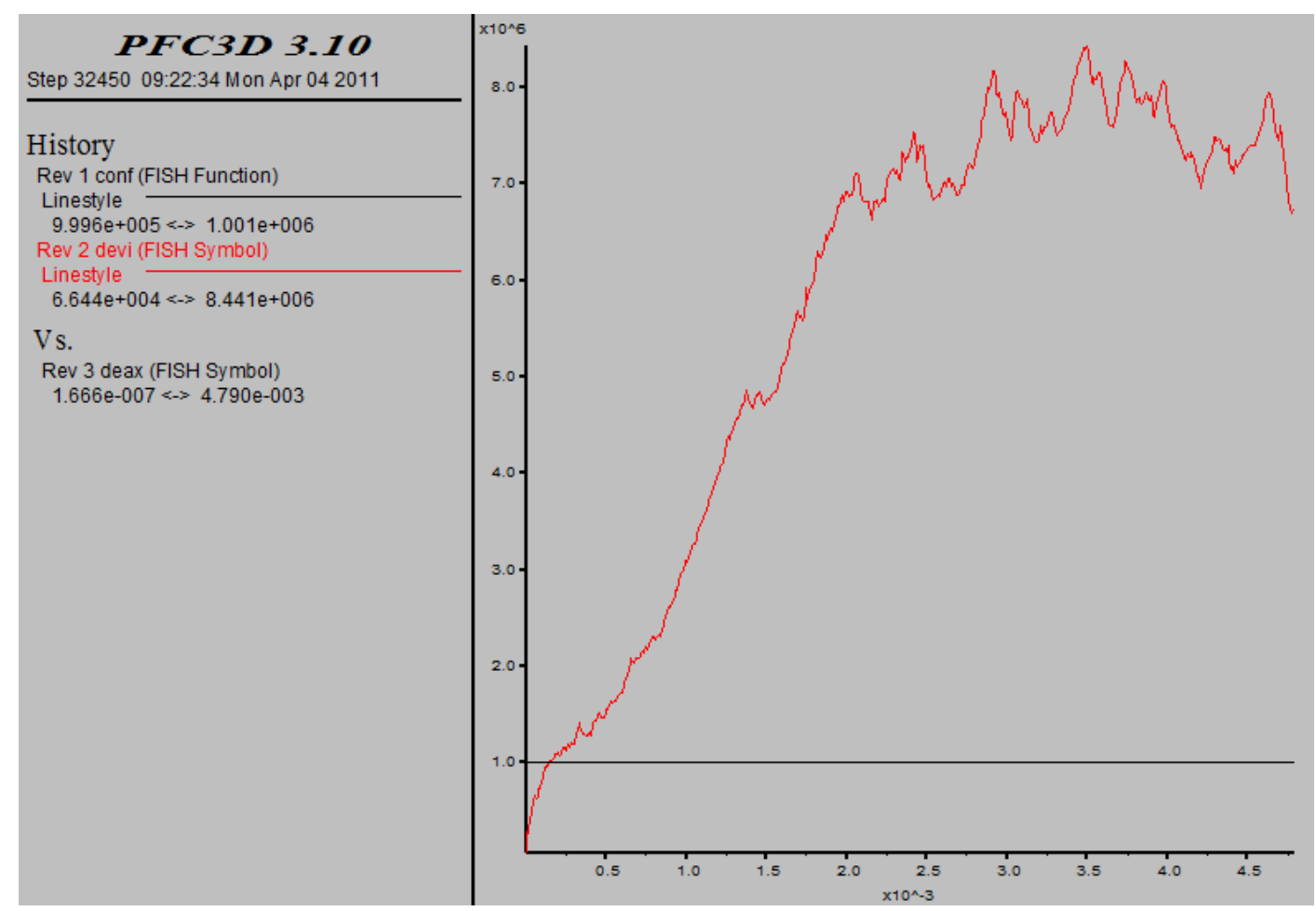

Figure 10. Stress-strain curve of the modeled formation specimen.

\subsubsection{Particle Invasion Modeling}

Transport and deposition of small particles in a porous medium is a complicated process which depends on the structure of the porous medium and the nature of the fluidrock interaction and particle-rock interaction. Various continuum approaches have been proposed by previous researchers to model the infiltration process in porous media; however, there are two main limitations of continuum approach: 1) the dynamics of filtration occurring at the pore scale is very difficult to be modeled by continuum method; 2) the mounding and clogging of pores cannot be represented by analytical models.

In this study, discrete element method is used to simulate the invasion of the particles. To better represent physics of the particles invasion process, we considered both particle-fluid interaction and particle-formation grain interaction in our model. 


\section{Particle-fluid interaction modeling}

Because of the complexity of modeling fluid flow in $\mathrm{PFC}^{3 \mathrm{D}}$, a simplified approach is proposed to model the interaction between fluid and invading particles. The hydrodynamic force acted on an invading particle exerted by the drilling fluid is simplified as the drag force, which can be calculated from the relative velocity between the fluid and the suspended particle and the size of the particle via following equation:

$$
\mathrm{F}_{\text {drag }}=6 \times \pi \times \mu \times \mathrm{r} \times\left(\mathrm{V}_{\text {fluid }}-\mathrm{V}_{\text {particle }}\right)
$$

where $\mu$ is the viscosity of the drilling fluid;

$r$ is the radius of the suspended particle;

$\mathrm{V}_{\text {particle }}$ is the velocity of the suspended particle;

$\mathrm{V}_{\text {fluid }}$ is the interstitial fluid velocity, which is calculated from the fluid velocity divided by porosity.

The fluid velocity is estimated from the permeability of the formation and the pressure difference between wellbore and the formation via Darcy's equation:

$$
\mathrm{q}=\frac{\mathrm{kA}}{\mu} \frac{\Delta \mathrm{P}}{\mathrm{L}}
$$

Two assumptions are made in our model:

1. The magnitude of the fluid velocity is constant.

At the initial stage of the fluid invasion, the magnitude of the fluid velocity is high and constant. As particle deposition goes, the permeability of the formation drops, which decreases the fluid velocity gradually. However, this factor is not considered in our model. Time-dependent fluid velocity could be applied in a future study.

2. The invading fluids flow in one direction. 
In real situation, the invading fluids change flow direction slightly when they hit obstacles, which are formation grains. Consequently, the direction of drag force also changes. Our assumption of the fluid flowing in a constant direction may cause unrealistic results of the simulation. Thus, proper adjustments need to be made, as discussed in details of the results session. Due to interaction with the formation grains, the particle velocities are changing continuously, so that the drag forces also change.

Besides the drag forces, drilling fluids also exert buoyant forces on suspended particles. As showing in Figure 11, a suspended particle is under the action of three forces: buoyant force, drag force and gravitational force. Compared to the drag force, the gravitational force and the buoyant force are rather small.

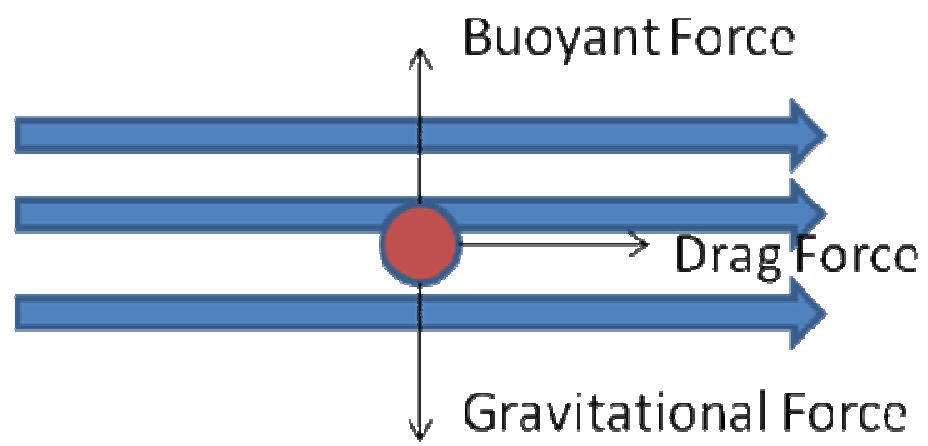

Figure 11. Three forces acts on a suspended particle: fluid drag force, buoyant force and gravitational force.

\section{Particle-grain interaction modeling}

The interaction between an invading particle and a formation grain is simulated in $\mathrm{PFC}^{3 \mathrm{D}}$ as interaction between two rigid balls. Our approach models the mechanical behavior of invaded particles which is often ignored in previous studies, although it simplifies the particle-fluid interaction when particles collide with formation grains. Figure 12 illustrates the difference between previous study and this study on modeling 
particle-grain interaction. Yellow balls in the figure indicate the next possible position of the invading particle, which collides with a formation grain. In previous studies, it is often assumed that a particle either is deposited on the grain surface or continues transporting with the fluid on the contact with a formation grain. While in this study, it is assumed that both invading particles and formation grains are rigid solids. When they are in contact, then contact model described in the previous section is used to predict the movement of the particles and grains.
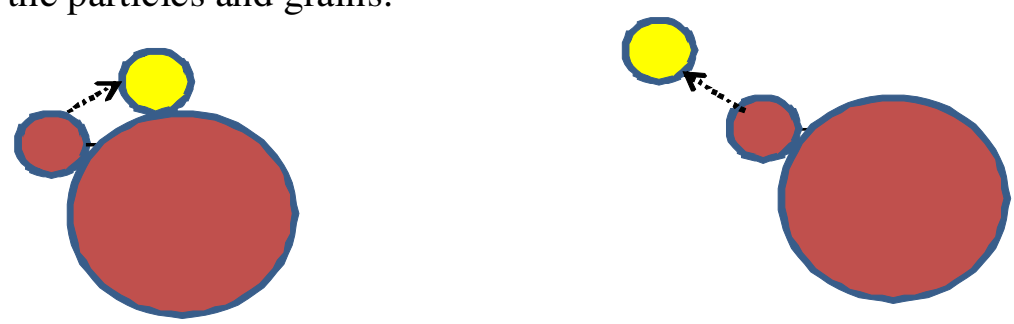

Figure 12. Different models of the particle-grain interaction. In the left diagram, the particle continues to travel along the fluid trajectory when it comes into contact with the formation grain. This interaction behavior was modeled in previous studies. In the right diagram, the movement of the particle is resulted from the contact force between the formation grain and the particle. This interaction behavior is modeled in this study.

In addition to the mechanical interaction between particles and formation grains, various other forces also act between particles and grain surface, such as electrostatic forces, dispersion forces and born repulsion forces. These forces are more crucial when the particle size is very small. Since the particle sizes considered in this study is much larger than 25 microns, we ignore these forces in the study.

According to the $\mathrm{PFC}^{3 \mathrm{D}}$ simulation theory, deposition of an invaded particle happens when the external forces acting on the particle reach equilibrium status.

\section{Particle and fluid properties}

Relative sizes of invading particles and formation pores/throats play an important role in formation damage processes. Particles which are bigger than the pore throats will not enter the porous medium. Within the porous medium, particles smaller than the pore 
throats penetrate into the pore throats and are possibly deposited there. The deposited particles impair the permeability of the formation. Therefore, for specific formation pore sizes and structure, mud particle size should be carefully chosen to reduce or control the permeability impairment. Suri and Sharma [19] measured the particle size inside the drillin fluids and reported that the particle size distribution was $0.7-800$ microns. In this study, several invading particle sizes are chosen to simulate the particle invasion process and to study the effects of the particle sizes on the formation damage. Mechanical properties of particles also affect the formation damage process, since they determine the interaction between particles and formation grains. The values of these properties are chosen in this study according to the published data by Mondol et al. in 2008 [20].

In addition to particle properties, properties of drilling fluids are also vital to the formation damage process, because the viscosity and velocity of the fluids determine the hydrodynamic drag forces exerted on the particles. Fluid velocity depends on pressure difference between borehole and formation and formation permeability. Typical overbalance pressure during drilling is $3.5 \mathrm{MPa}(500 \mathrm{psi})$, which is used in this study. The permeability estimated from the porosity and grain size according to the Carman-Kozeny equation is used to calculate the fluid velocity.

The properties of invading particles and fluid are defined in Table 2. 
Table 2. Fluid and Particle Properties

\begin{tabular}{|c|c|}
\hline Input Parameters & Value \\
\hline Particle Size $(\mathrm{mm})$ & $0.025-0.1$ \\
\hline Particle Density $\left(\mathrm{kg} / \mathrm{m}^{3}\right)$ & 2146 \\
\hline Particle Normal Stiffness $(\mathrm{GPa})$ & 63 \\
\hline Particle Shear Stiffness $(\mathrm{GPa})$ & 26 \\
\hline Particle Concentration & $10 \%$ \\
\hline Fluid Density $\left(\mathrm{kg} / \mathrm{m}^{3}\right)$ & 1095 \\
\hline Fluid viscosity $(\mathrm{cp})$ & 50 \\
\hline Overbalance pressure during drilling $(\mathrm{MPa})$ & 3.5 \\
\hline
\end{tabular}

\section{Simulation setup}

$\mathrm{PFC}^{3 \mathrm{D}}$ is used to simulate the particle invasion process. In this study, static mud invasion is assumed, i.e. shear force exerted by fluid circulation is not considered. Figure 13-(A) illustrates the simulation setup for the model. Invading particles, which are represented by small gray balls in the figure, are generated besides the formation specimen. Under the action of hydro-dynamic forces, particles start to invade into the formation matrix. All the particles have the same initial velocity, which is equal to the fluid velocity. After particles enter the formation sample, they start to interact with formation grains. The contact forces between particles and formation grains change the direction and magnitude of the particle velocities; consequently change the drag forces exerted by the fluid. Therefore, after certain computation cycles, the drag forces are recomputed according to the current particle velocities.

The extent of formation damage depends on how long the formation is exposed to the drilling fluids. In our simulation, we assume that the formation is exposed to the fluids long enough that the invasion process stops when external mud cake builds up at the formation face and no more particles can invade into the formation matrix. 


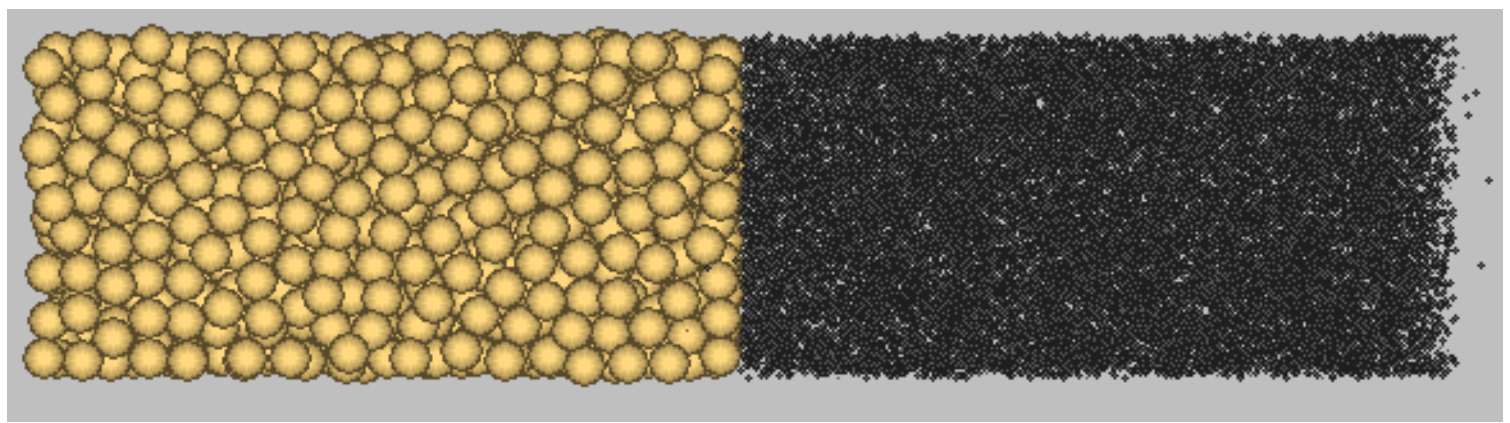

(A)

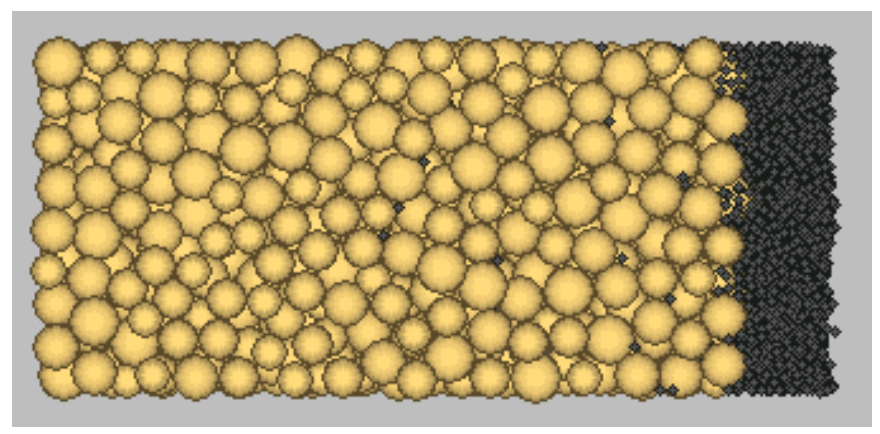

(B)

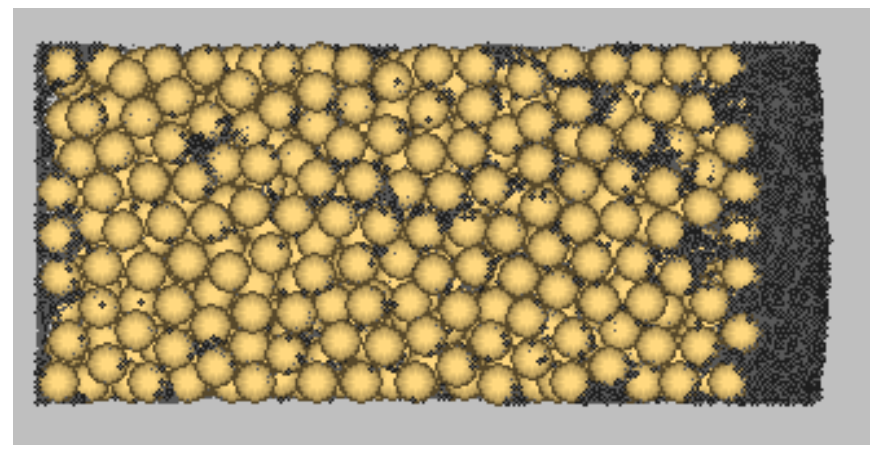

(C)

Figure 13. Simulation of particle invading into formation matrix. The big ball assembly represents formation specimen, and the small gray balls represent invading particles. (A) shows simulation setup. (B) and (C) indicate invasion results. In figure (B), formation has initial porosity as $30 \%$, and particles are three times smaller than formation grains. In figure(C), initial porosity of formation is $40 \%$, and particles are ten times smaller than formation grains. 


\subsection{RESULTS AND DISCUSSION}

The extent of formation damage is estimated through porosity reduction and invasion depth in this chapter. The permeability impairment will be studied in the next chapter.

\subsubsection{Effects of Drag Forces}

The simulation results of the selected properties are shown in Table 3.

Table 3. Input Parameters and Results of the Simulation

\begin{tabular}{|c|c|c|c|c|}
\hline \multicolumn{3}{|c|}{ Input Parameters } & \multicolumn{2}{c|}{ Results } \\
\hline Initial & Particle & Particle & Damaged & Invaded \\
Porosity & Size & Size/Grain Size & Porosity & depth \\
\hline $30 \%$ & $0.025-0.05$ & $1 / 5-1 / 10$ & $29.6 \%$ & $1.65 \mathrm{~mm}$ \\
\hline
\end{tabular}

Compared to the published experimental results by Oort et al. [9], the amount of the invaded particles is small and the particle invasion depth of the simulation is rather shallow. One possible reason is that our assumption of a constant fluid velocity is over simplified. In reality, fluid flow changes its direction when it hits obstacles, which are formation grains in our case; and thus the directions of drag forces exerted on the particles are also changed. Consequently, the suspended particles travel deeper into the formation matrix. To reduce the adverse effects caused by this assumption, bigger drag forces are applied to particles, as shown in Figure 14. 


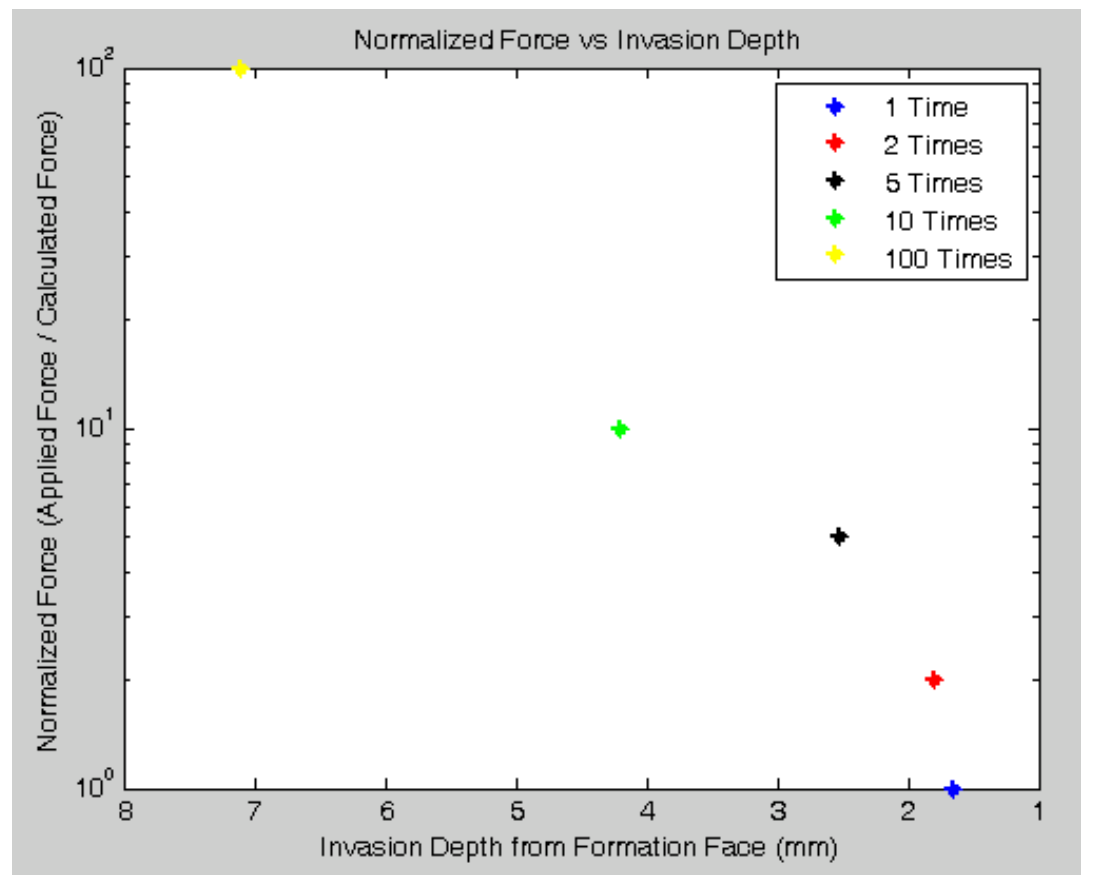

Figure 14. Normalized drag forces vs invasion depth

Clearly, bigger drag forces result in deeper particle invasion; and the result of the largest applied force in the figure is the closest to the experimental data. Therefore, we use large drag forces to generate the damaged formation specimens and then study the effects of initial porosities and particle sizes on formation damage.

\subsubsection{Effects of Initial Formation Porosities and Particle Sizes}

Different initial formation porosities and different particle sizes are used to generate the damaged formation specimens.

Table 4 shows the properties of the formation specimens and the invading particles and the results of the formation damage. In general, higher porosity and bigger size difference between particles and formation grains result in more porosity reduction and deeper invasion. 
Table 4. Numerical simulation settings and results of particle invasion. The formation specimen is represented by an equal size sphere packing, and the particle size is uniform.

\begin{tabular}{|l|c|c|c|c|c|}
\hline & \multicolumn{3}{|c|}{ Input Parameters } & \multicolumn{2}{c|}{ Results } \\
\hline & $\begin{array}{c}\text { Initial } \\
\text { Porosity }\end{array}$ & $\begin{array}{c}\text { Particle } \\
\text { Size }\end{array}$ & $\begin{array}{c}\text { Particle } \\
\text { Size/Grain Size }\end{array}$ & $\begin{array}{c}\text { Damaged } \\
\text { Porosity }\end{array}$ & $\begin{array}{c}\text { Invaded } \\
\text { depth (mm) }\end{array}$ \\
\hline Specimen 1 & $30 \%$ & 0.16 & $1 / 3$ & $29 \%$ & 4 \\
\hline Specimen 2 & $30 \%$ & 0.1 & $1 / 5$ & $23 \%$ & 8 \\
\hline Specimen 3 & $30 \%$ & 0.05 & $1 / 10$ & $22 \%$ & 8 \\
\hline Specimen 4 & $40 \%$ & 0.16 & $1 / 3$ & $33 \%$ & 8 \\
\hline Specimen 5 & $40 \%$ & 0.1 & $1 / 5$ & $32 \%$ & 8 \\
\hline Specimen 6 & $40 \%$ & 0.05 & $1 / 10$ & $31 \%$ & 8 \\
\hline
\end{tabular}

Detailed analysis on the damaged formation specimens are conducted in the following sections.

\subsubsection{Particle Invasion Results of Specimen 1}

Figure 13-B shows the simulation result of particles invading into formation specimen 1 . The initial porosity of the specimen is about $30 \%$, and the particles are three times smaller than the formation grains. Clearly, most particles are located at the entrance of the specimen. As invasion proceeds, the internal mud cake forms first, which prevents further particle invasion into the deep formation, and then the low permeability external mud cake forms at the formation face. The formed external mud cake stops the loss of drilling fluids. Consequently, the particle invasion process stops.

In this damaged formation sample, a total of 704 small particles invaded into the formation specimen, and almost $70 \%$ particles are located at the near formation face region as shown in Figure 15. Fewer than 5\% particles invaded into the formation 
specimen deeper than $0.5 \mathrm{~mm}$. This figure also indicates that the invasion depth is about $4 \mathrm{~mm}$, since the percentage of the invaded particles goes to zero when it is deeper than $4 \mathrm{~mm}$.

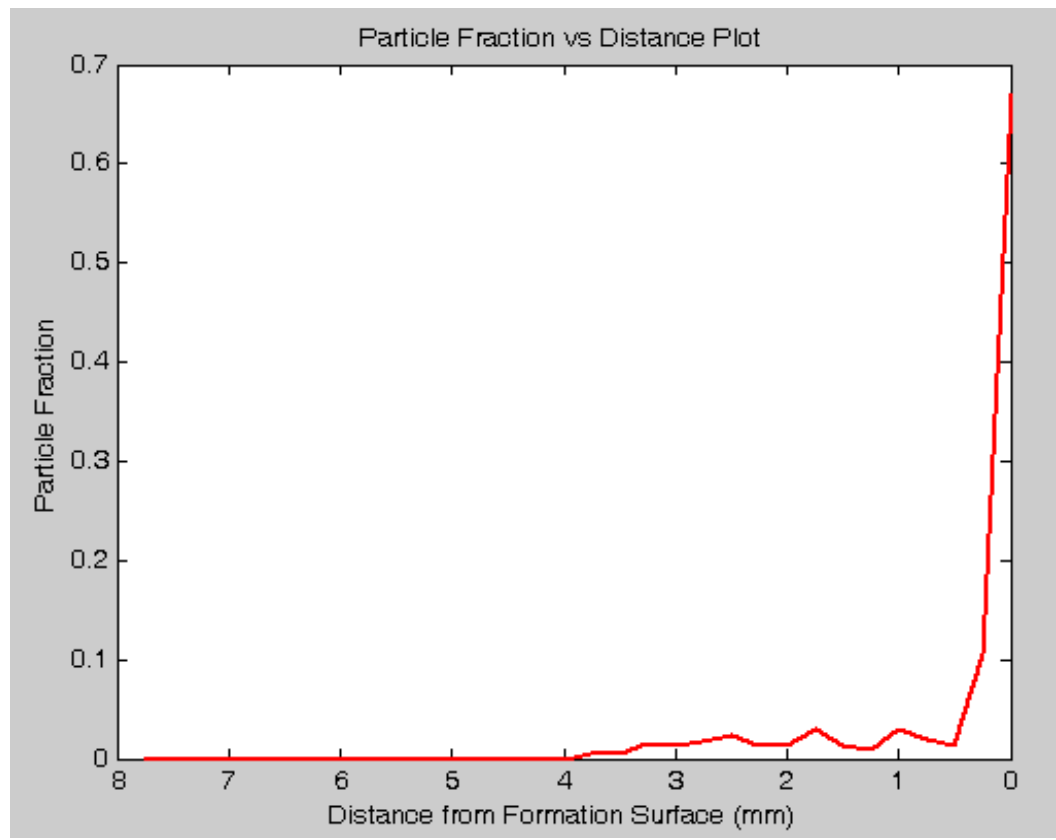

Figure 15. Fraction of invaded particles along the distance from the formation face. The formation specimen has initial porosity $30 \%$, and the size of invaded particles is three times smaller than that of formation grains.

The total porosity of the formation specimen is reduced by $1 \%$. More detail porosity reduction along the distance from the formation face is examined in Figure 16. At the near formation face region, the porosity reduction is about $5 \%$. As it goes deeper into the formation, the porosity reduction decreases and reaches zero at the depth of $4 \mathrm{~mm}$, where invasion stops. Because the size difference between the formation grains and the invaded particles is small, particles cannot invade into most of the pores, and thus the amount of invaded particles is rather small. The permeability impairment caused by the invaded particles will be estimated in the next chapter. 


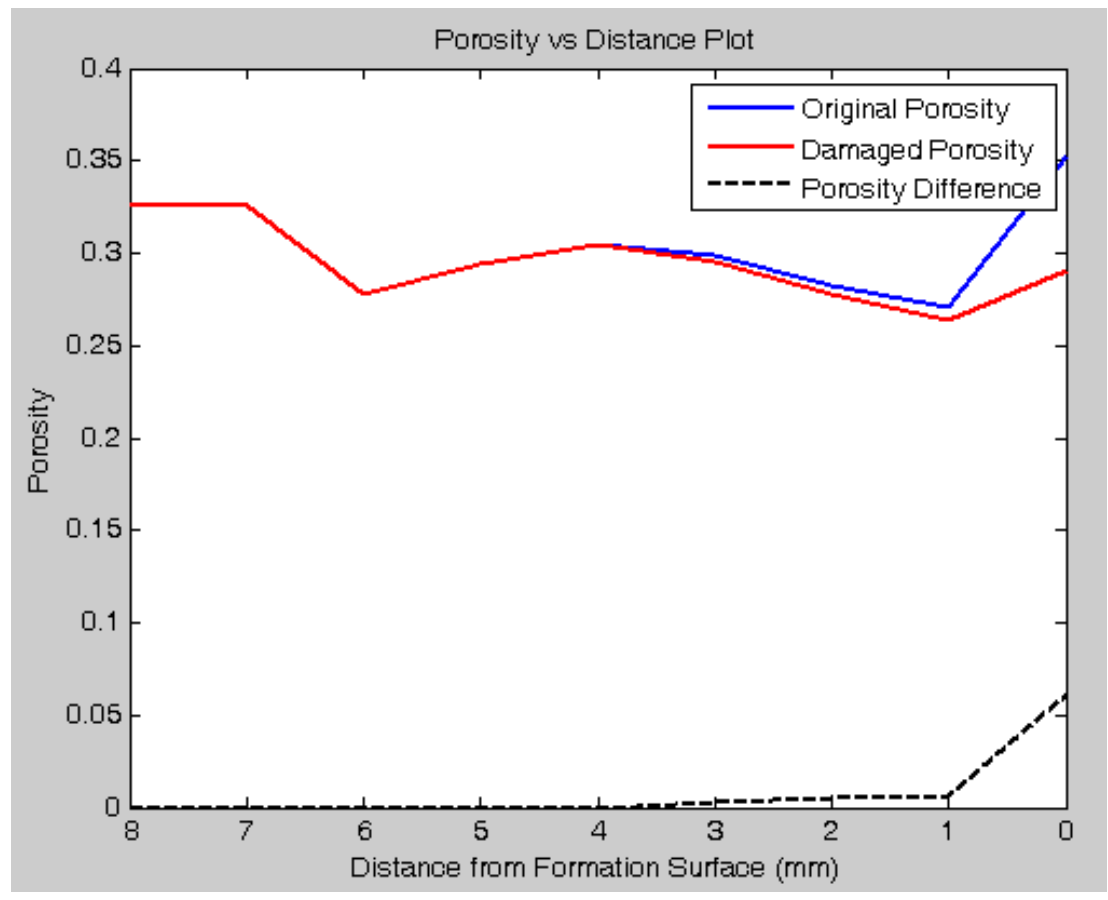

Figure 16. Formation porosity reduces due to the particles invasion. The original formation porosity is about $30 \%$ and the particle size is 3 times smaller than the formation grain size.

\subsubsection{Particle Invasion Results of Specimen 5}

A higher initial porosity and a smaller invaded particle size result in more severe particle invasion. Specimen 5 has initial porosity of $40 \%$ and the size of invaded particles is about $0.1 \mathrm{~mm}$, which is five times smaller than the formation grain size. Compared to specimen 1, this damaged formation specimen contains a bigger amount of invaded particles. The total porosity reduction due to particle invasion is also higher, which is about $8 \%$.

Figure 17 illustrates the percentage of invaded particles along the distance from the formation face. In comparison to Specimen 1, the fraction of particles near the formation face is much lower, about $10 \%$, which indicates invaded particles are more evenly distributed in the formation matrix. The figure also shows that although the invasion depth is $8 \mathrm{~mm}$, there are very few particles invaded deeper than $4 \mathrm{~mm}$. 


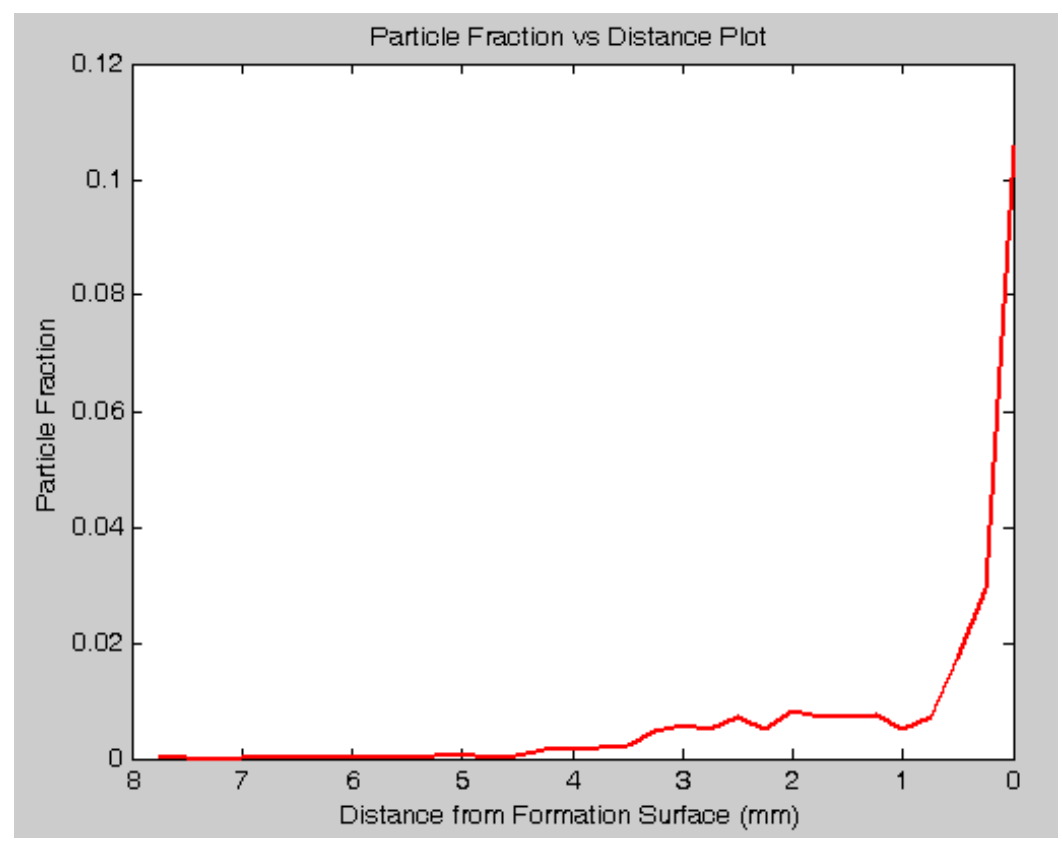

Figure 17. Fraction of invaded particles along the distance from the formation face. The formation specimen has initial porosity of $40 \%$, and invaded particles are five times smaller than formation grains.

The plot of porosity difference between the original formation sample and the damaged formation sample (see Figure 18) shows the same trend as Specimen 1. The difference decreases from the formation face to the deep region of the formation matrix. The porosity reduction at the formation face of this specimen is 2 times bigger than that of Specimen 1, which is caused by either the higher porosity or the smaller particle size. 


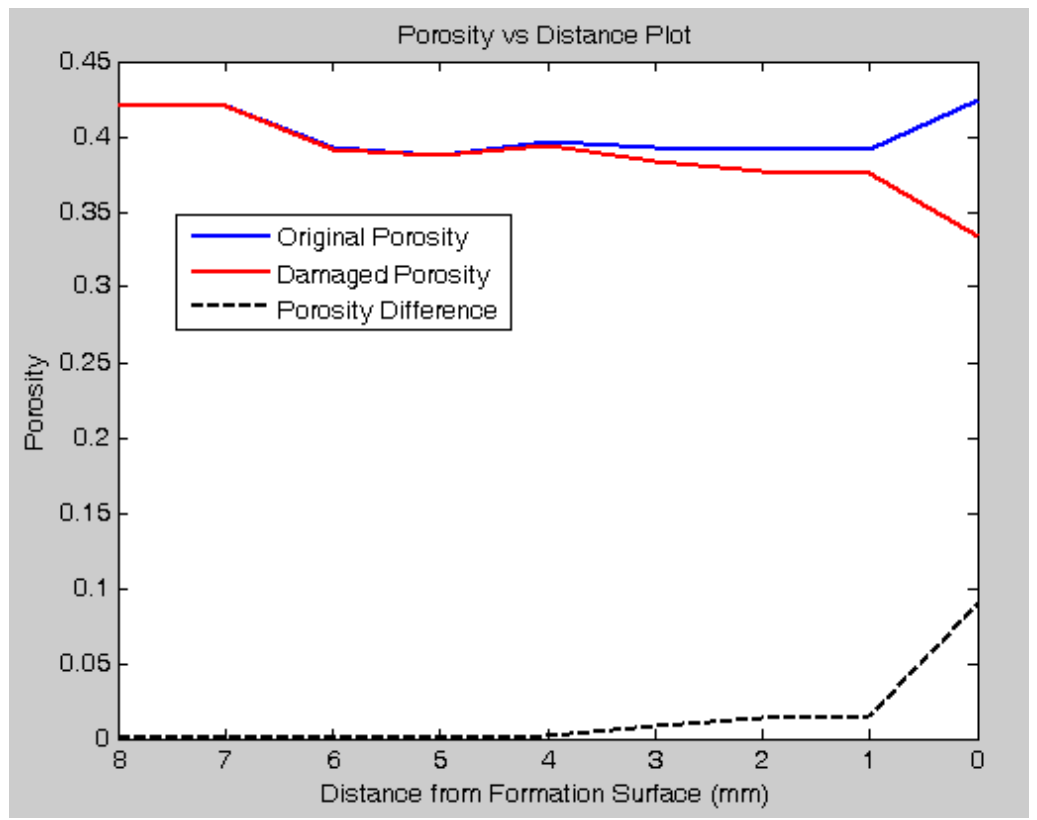

Figure 18. Formation porosity reduces due to particles invasion. The original formation porosity is about $40 \%$ and the particle size is five times smaller than the formation grain size.

\subsubsection{Particle Invasion Results of Specimen 6}

Specimen 6 has the same porosity as Specimen 5, which is $40 \%$, while the invaded particle size is smaller, which is ten times smaller than the formation grain size.

Figure 19 shows the percentage of invaded particles along the distance from the formation face. The whole trend is very similar to Specimen 5, but compared to Specimen 5, invaded particles are more evenly distributed in the formation matrix. Moreover, more particles invaded into the formation deeper than $4 \mathrm{~mm}$, which indicates that finer particles invade deeper. 


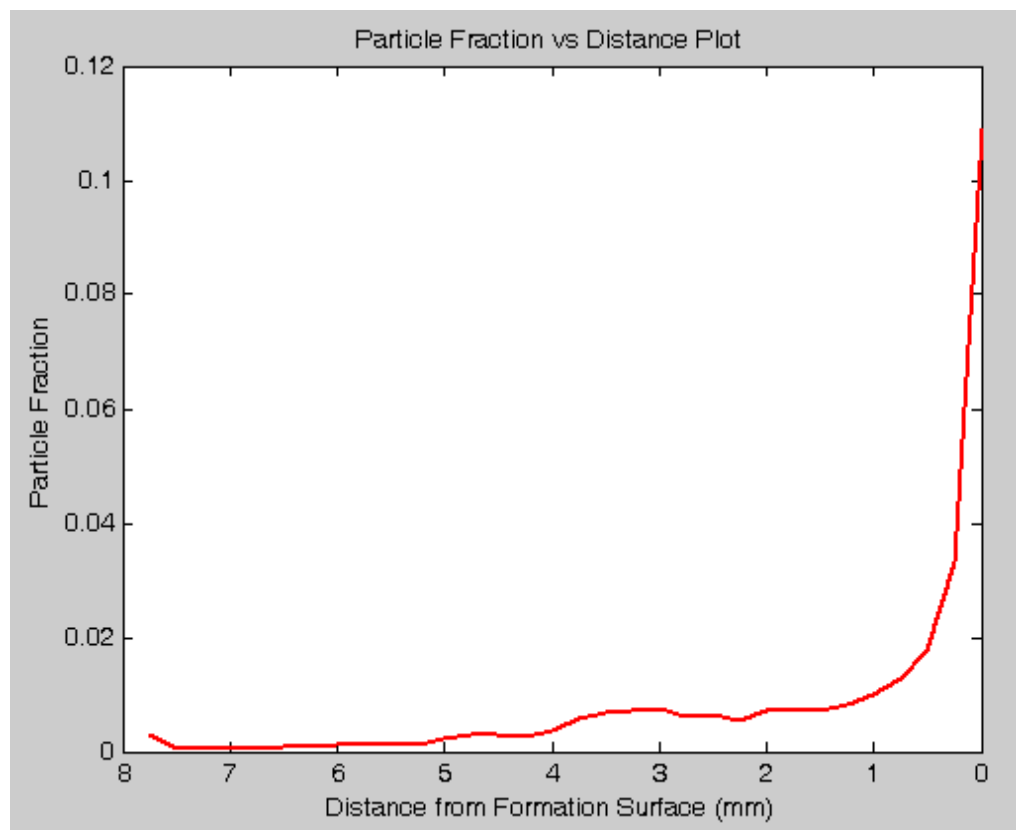

Figure 19. Fraction of invaded particles along the distance from the formation face. The formation specimen has initial porosity of $40 \%$, and the size of invaded particles is ten times smaller than that of formation grains.

Figure 20 shows the porosity reduction due to particle invasion, which has the same trend as that of Specimen 5. The porosity reduction values at different locations of this specimen are also very close to those of Specimen 5 . This shows that if the size ratio of particles and formation grains is small enough, the invaded solid volume remains constant. 


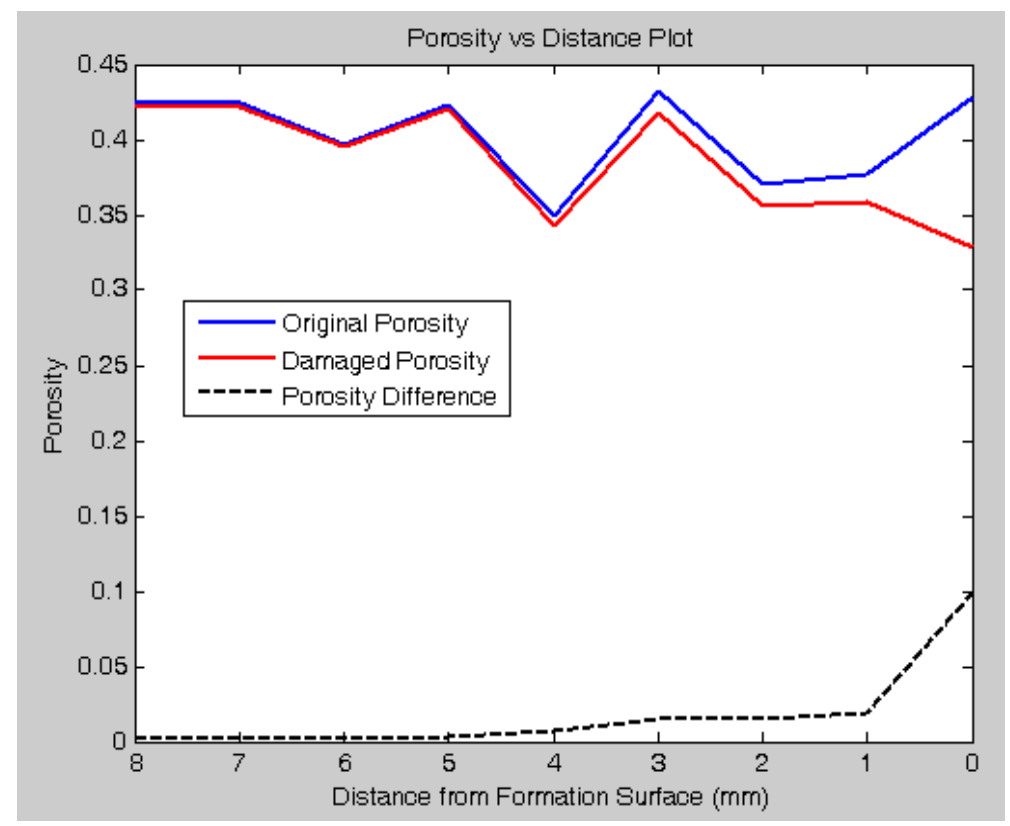

Figure 20. Formation porosity reduces due to particle invasion. The original formation porosity is about $40 \%$ and the particle size is ten times smaller than the formation grain size.

\subsubsection{Specimen with Different Particle Sizes}

The uniform particle size distribution is used in the previous samples to study the effect of the particle size on formation damage. The particle size distribution is an important factor of mud cake build up time and permeability, which in turn affect the severity and extent of formation damage. In reality, drilling fluids contain solids with various sizes. To simulate the real situation and study the effect of the particle size distribution, a new specimen is generated with properties listed in Table 5. The porosity reduction due to particle invasion of this specimen is the same as that of Specimen 3, although the mean particle size of this specimen is smaller than that of Specimen 3. The particle fraction and porosity reduction along the distance from the formation face of this specimen has the same trend as Specimen 3. Therefore, under the condition of similar mean particle size, a uniform size distribution and a medium range size distribution have similar formation damage results. 
Table 5. Numerical simulation results of particle invasion. The formation specimen is represented by an equal size sphere packing, and the particle size is 5-10 times smaller than formation grain size.

\begin{tabular}{|c|c|c|c|c|c|}
\hline & \multicolumn{3}{|c|}{ Input Parameters } & \multicolumn{2}{c|}{ Results } \\
\hline & $\begin{array}{c}\text { Initial } \\
\text { Porosity }\end{array}$ & $\begin{array}{c}\text { Particle Size } \\
(\mathbf{m m})\end{array}$ & $\begin{array}{c}\text { Particle } \\
\text { Size/Grain Size }\end{array}$ & $\begin{array}{c}\text { Damaged } \\
\text { Porosity }\end{array}$ & $\begin{array}{c}\text { Invaded depth } \\
(\text { mm })\end{array}$ \\
\hline $\begin{array}{c}\text { Specimen } \\
7\end{array}$ & $30 \%$ & $0.025-0.05$ & $1 / 5-1 / 10$ & $22 \%$ & 8 \\
\hline
\end{tabular}

Figure 21 shows only invaded particles. The red balls represent particles with size in the range of $0.045-0.05 \mathrm{~mm}$, and the size range of black balls is $0.025-0.03 \mathrm{~mm}$. Clearly, in the shallow region of the formation specimen, the amount of big particles surpasses small particles, while in the deep region, there are more small ones.

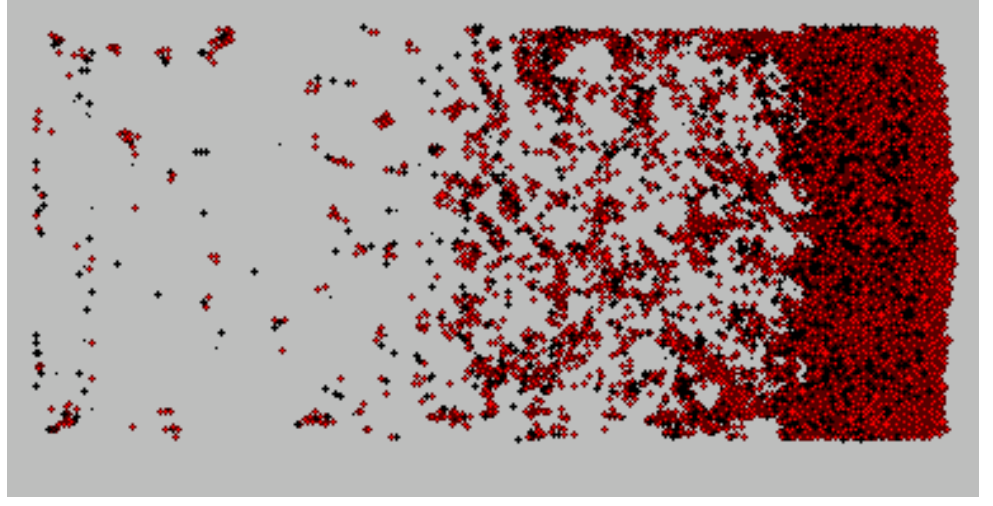

Figure 21. Invaded particles in Specimen 7. Red balls represent particles with size in the range of $0.045-0.05 \mathrm{~mm}$; and the size range of black balls is $0.025-0.03 \mathrm{~mm}$.

\subsubsection{Specimen with Different Formation Grain Sizes}

The network model introduced in the next chapter is best suitable for equal size sphere packing, thus, in this study, the grain size of previous specimens is uniform. However, for the real sample, both formation grains and invading particles have certain size distributions. Moreover, the particle invasion simulation is not limited by the size distribution of formation grains. To better represent the real situation, the size distribution 
of Antler sandstone is adopted to simulate the particle invasion. Table 6 shows the specimen generated from certain grain size distribution and invading particle size distribution.

Table 6. Numerical simulation results of particle invasion. The grain sizes of the sphere packing are in the range of $0.15-0.3 \mathrm{~mm}$.

\begin{tabular}{|c|c|c|c|c|c|}
\hline & \multicolumn{3}{|c|}{ Input Parameters } & \multicolumn{2}{c|}{ Results } \\
\hline & $\begin{array}{c}\text { Initial } \\
\text { Porosity }\end{array}$ & $\begin{array}{c}\text { Particle } \\
\text { Size }\end{array}$ & Particle Size & $\begin{array}{c}\text { Damaged } \\
\text { Porosity }\end{array}$ & $\begin{array}{c}\text { Invaded } \\
\text { depth }\end{array}$ \\
\hline Specimen 8 & $35 \%$ & $0.15-0.3$ & $0.025-0.05$ & $27 \%$ & $8 \mathrm{~mm}$ \\
\hline
\end{tabular}

The porosity reduction along the distance from formation surface of this specimen is also calculated and plotted in Figure 22.

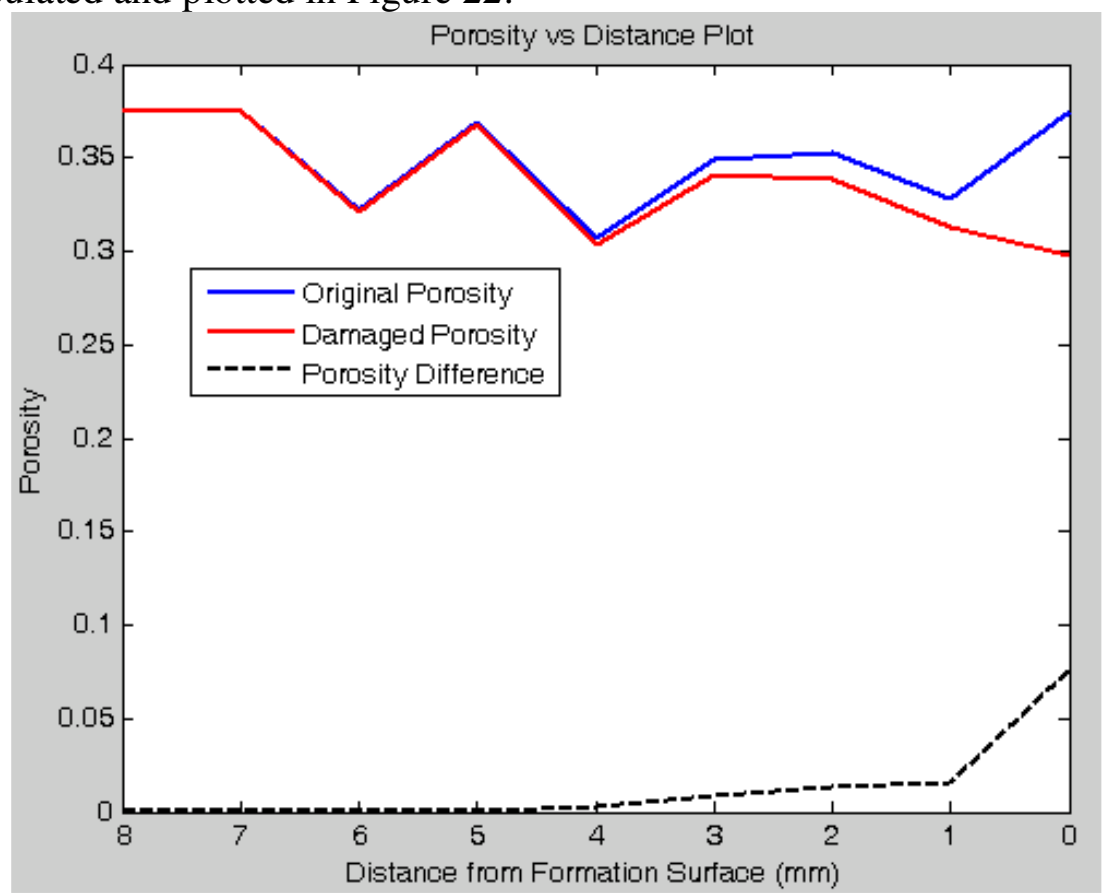

Figure 22. Formation porosity reduces due to the particles invasion. This plot is generated from Specimen 8.

From the investigation of the resultant porosity damage, it seems that sorting of formation grains does not affect the particle invasion process to any large extent; while 
grain size sorting affects pore structures of a porous medium, which is discussed in detail in the next chapter.

Besides the factors of initial formation porosities and size distributions of formation grains and particles, other factors, such as fluid viscosities and circulation velocities, also affect the invasion process, which are not studied in this thesis study. 


\section{Pore Network Modeling of Granular Media}

\subsection{NETWORK MODEL OVERVIEW}

A network model was first invented by Fatt [21] to study the connections between pores in a porous medium. The connections are modeled as a collection of intersecting tubes, which represent void space within the medium. Network models can explain various phenomena in porous media, such as entry pressure, residual saturation, permeability and resistivity (for overview see [22]). The real geometry of the pore structure cannot be represented properly using regular lattices such as in the work of Fatt. Modern network models use a physically representative network. For granular media specifically, topology and geometry is derived directly from grain locations [23-25]; and Delaunay tessellation method [26] is used to find pores and pore throats in a network. Further, representative network models can be derived from imaged consolidated porous media [27], [28], but those methods are not applicable to our samples.

The network model related terms, such as Delaunay tessellation, pore body and pore throat, are introduced first in this chapter.

\subsubsection{Delaunay Tessellation}

Delaunay tessellation is a mathematical method, which takes a series of discrete points as input which then become vertices of triangles (in 2D) or tetrahedral (in 3D) that completely cover the space. The method is readily available in standard software such as MATLAB (http://www.matlab.com), Octave (http//www.gnu.org/software/octave/) and Qhull (http://www.qhull.org). Figure 23 shows the 2D Delaunay tessellation of a disk pack. 


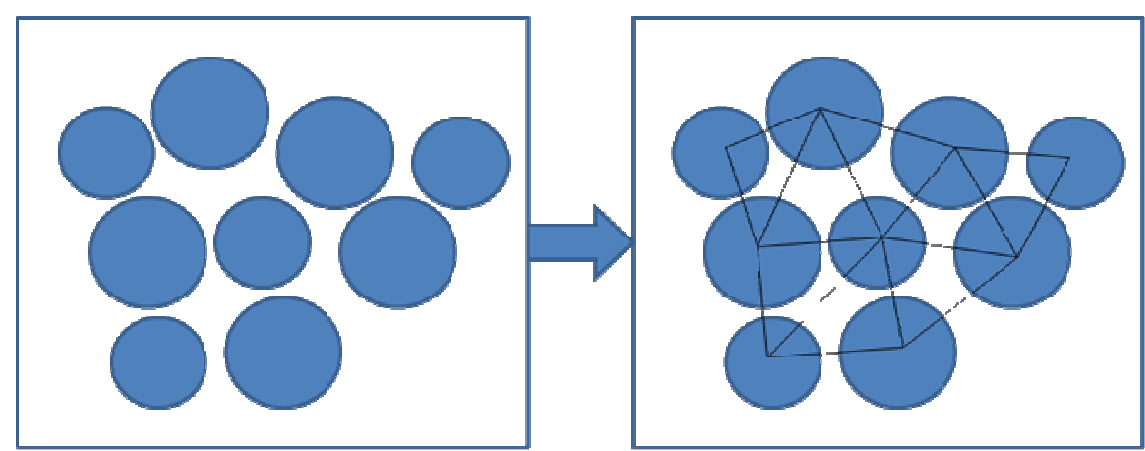

Figure 23. 2D Delaunay tessellation

Delaunay tessellation in $3 \mathrm{D}$ is to find a set of four closest points which form tetrahedrons. For a dense sphere pack, Delaunay tessellation generates a series of connected tetrahedra as shown in Figure 24.
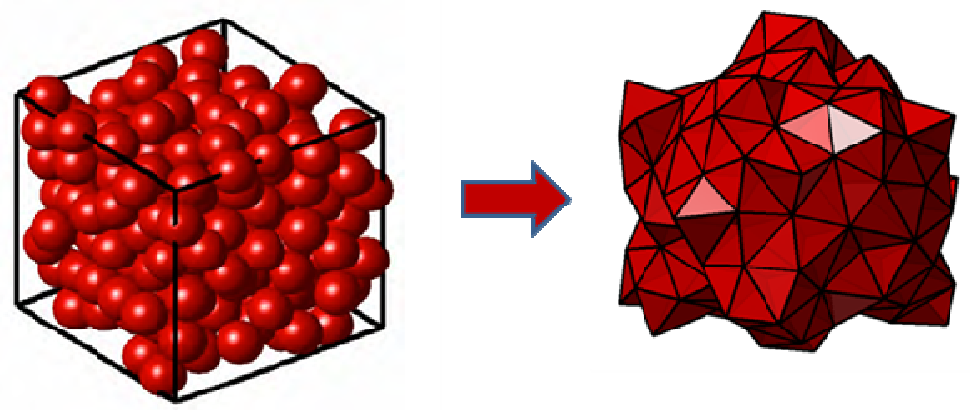

Figure 24. 3D Delaunay tessellation [29]. Tetrahedra vertices on the right correspond to sphere centers.

\subsubsection{Pore Throat Network Based on Delaunay}

Mason and Mellor in [30] introduced the Delaunay tessellation method into the network construction of sphere packs. Using Delaunay tessellation, a porous medium is meshed into interconnected cells, which are represented by tetrahedrons. A pore is defined as the center of the cell, and a pore body is the void space inside a triangle for 2D and a tetrahedron for $3 \mathrm{D}$. A pore throat refers to the path between two pores. In $2 \mathrm{D}$, it is the edge of the triangle, and in $3 \mathrm{D}$, it is the face of the tetrahedron. 


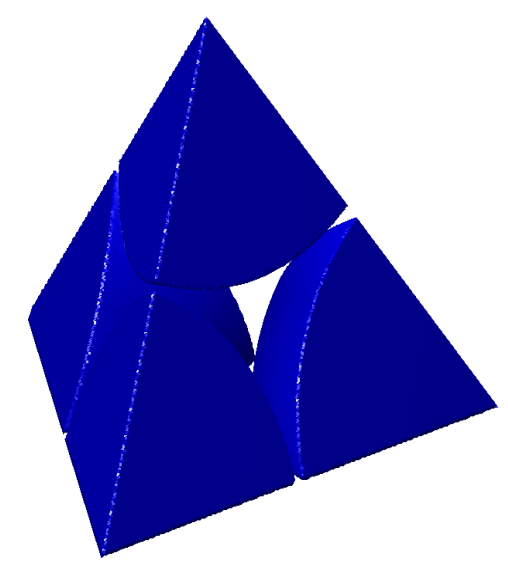

\section{Figure 25. Tetrahedron from Delaunay Tessellation}

Figure 25 shows a tetrahedron from Delaunay tessellation. Each pore connects to four other pores through the pore throats represented by the four faces of the tetrahedron. The face of the tetrahedron is the narrowest section of the fluid path that controls the flow resistance of the path. In our network model, a pore throat is considered as a cylinder tube, whose radius can be calculated from the flow area of the minimum section. Note that if relative permeability of the medium is to be studied, then it is better to use tube cross-sections with corners that are capable of retaining residual fluids [27], [31], [32]. The calculation of the actual flow area of the section is complicated and it is difficult to match the fluid-rock contacting surface with the cylinder's surface. Therefore, two kinds of radius are normally used for the flow calculation--inscribed radius and effective radius, as shown in Figure 26. Inscribed radius can be calculated from the inscribed circle, which is the circle just touching surfaces of the three solids. Effective radius is estimated according to the equivalent flow area method. To calculate the effective radius, the flow area of the face needs to be calculated first:

$$
\mathrm{S}_{\text {flow }}=\mathrm{S}_{\text {triangle }}-\mathrm{S}_{\text {solid }}
$$

The effective radius of the pore throat then can be calculated by the equation: 


$$
\mathrm{R}_{\text {effective }}=\sqrt{\frac{\mathrm{S}_{\text {flow }}}{\pi}}
$$

Clearly, effective radius is bigger than the inscribed radius.

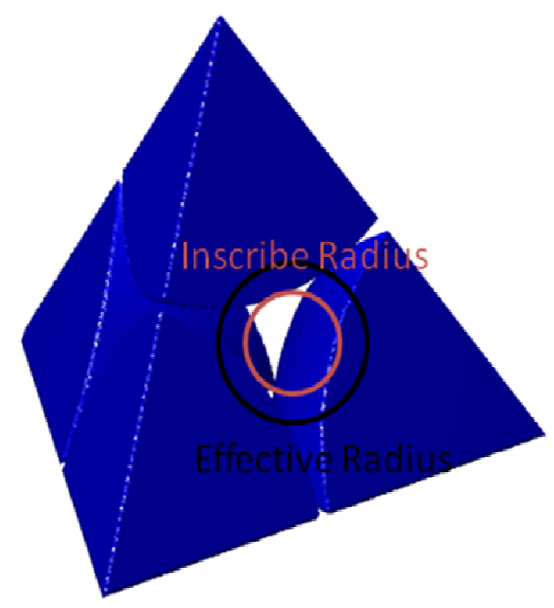

Figure 26. Two kinds of pore throat radius which can be used in network model calculation. Inscribe radius is obtained by finding the inscribe circle; and effective radius is calculated by equivalent flow area

The length of the pore throat is defined as the distance between the centers of two pores as showing in Figure 27. Note that Euclidian distance is taken between pore centers, while in reality the path is not necessarily a straight line.

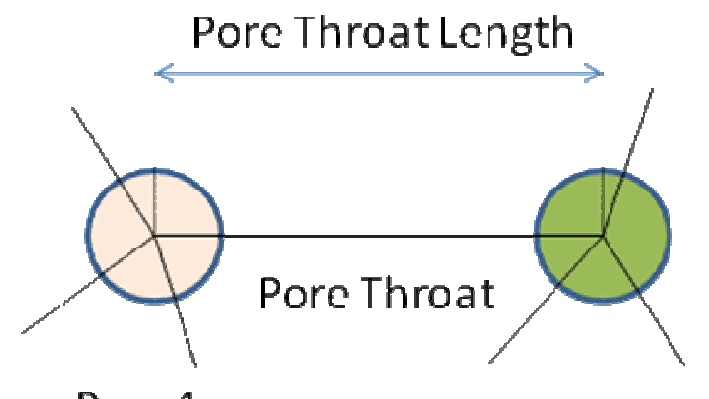

Pore 1

Figure 27. Pore Throat Length

In this study, the center of a pore in three dimensions is defined as the center of the inscribed sphere which is touching the four gains. However, in some cases, the found pore center is outside the tetrahedron defined by four grains, so an alternative method is 
used to calculate the center of the pore, which is to find the geometric center of the tetrahedron.

\subsubsection{Network Model of Transport}

Figure 28 shows the set up for fluid transport simulation of the porous medium. Single phase flow is considered in this study. The porous medium is assumed to be saturated with the fluid, and fluid flowing through the medium is assumed to be in steady state. A constant pressure difference is applied to the $\mathrm{z}$ direction, which is the flow direction. There is no fluid exchange in the lateral direction.

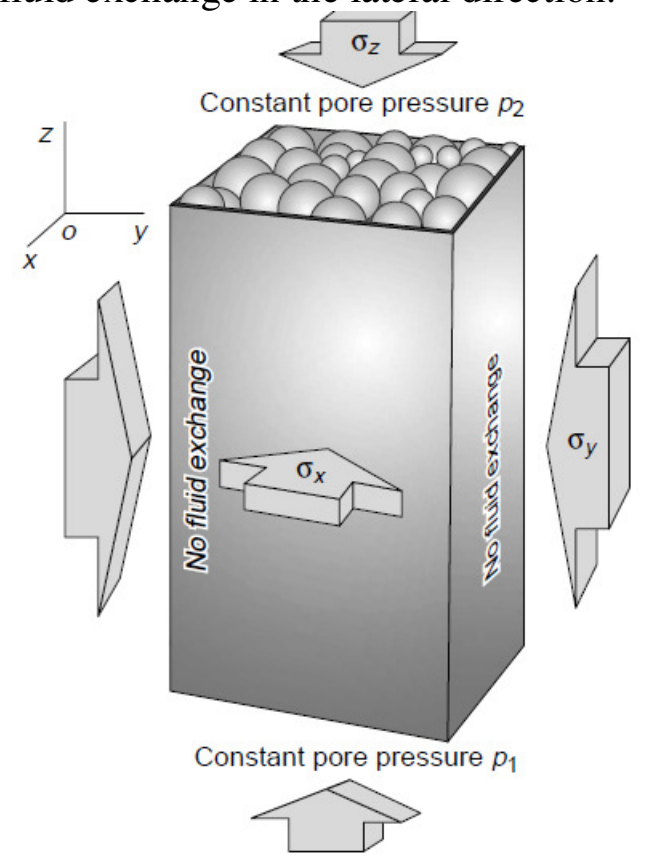

Figure 28. Setup for fluid transport simulation of the porous medium [8]

Figure 29 is an illustration of the network of pores and pore throats. Each edge in the lattice represents a pore throat and each intersection point represents a pore. The hydraulics conductance of each pore throat is calculated according to the HagenPoiseuille equation: 


$$
g_{h y d r}=\frac{\pi r^{4}}{8 \mu L}
$$

Where $\mathrm{r}$ is the radius of the pore throat and $\mathrm{L}$ is the length of the pore throat.

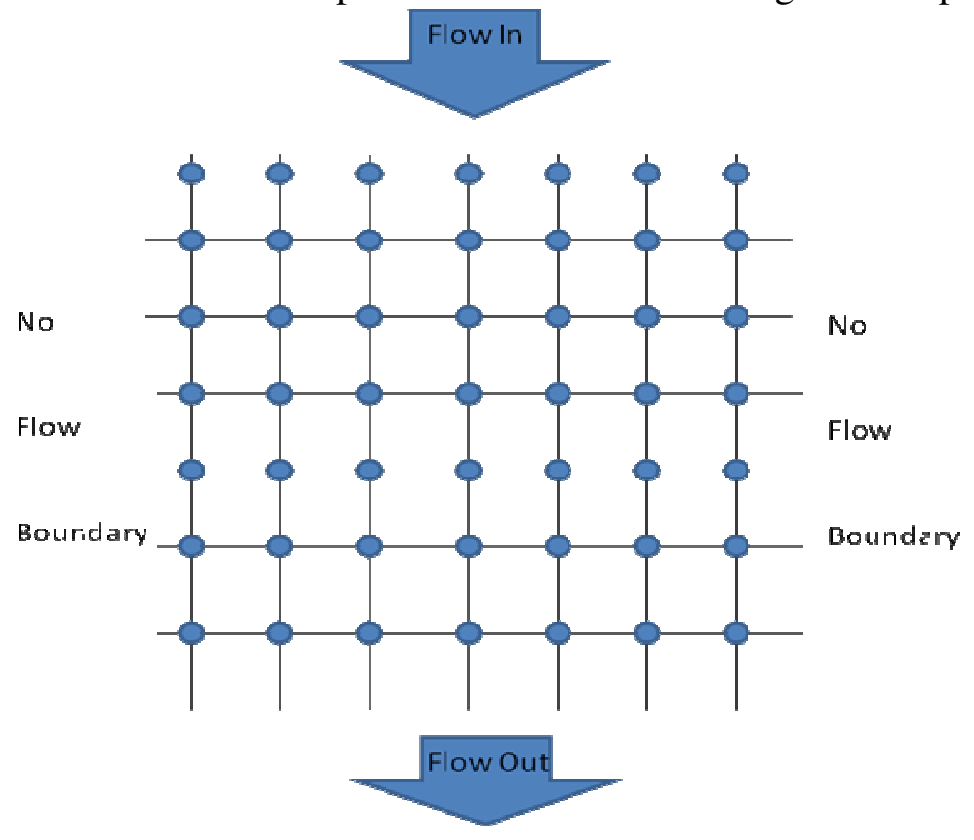

Figure 29. Network representation of the transport simulation

Furthermore, each node in the network connects to other four nodes by edges (see Figure 30). For a local node, the mass is conserved, that is the flux flowing into the node should be equal to the flux flowing out from the node.

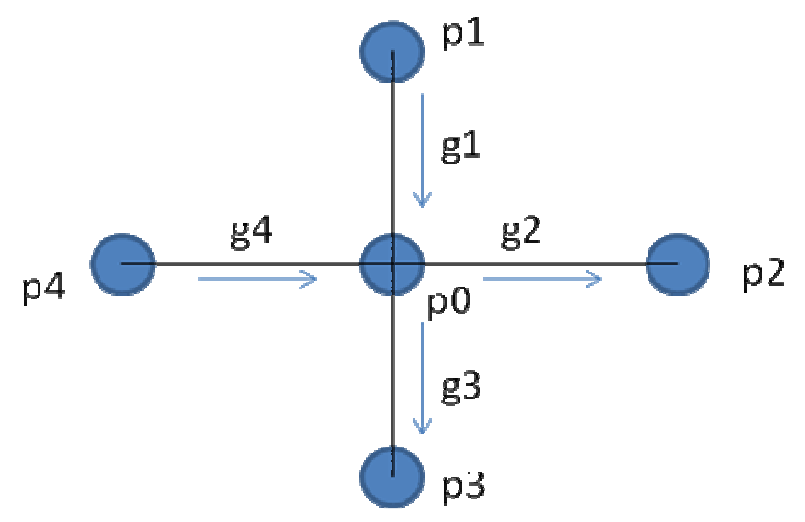

Figure 30. Mass conservation of the local node 
The flux at each edge can be calculated using the following equation:

$$
\begin{aligned}
& \mathrm{q}_{1}=\left(\mathrm{p}_{1}-\mathrm{p}_{0}\right) \times \mathrm{g}_{1} \\
& \mathrm{q}_{2}=\left(\mathrm{p}_{2}-\mathrm{p}_{0}\right) \times \mathrm{g}_{2} \\
& \mathrm{q}_{3}=\left(\mathrm{p}_{3}-\mathrm{p}_{0}\right) \times \mathrm{g}_{3} \\
& \mathrm{q}_{4}=\left(\mathrm{p}_{4}-\mathrm{p}_{0}\right) \times \mathrm{g}_{4}
\end{aligned}
$$

According to a mass balance:

$$
\sum \mathrm{q}_{\mathrm{i}}=\mathrm{q}_{1}+\mathrm{q}_{2}+\mathrm{q}_{3}+\mathrm{q}_{4}=0
$$

All the nodes in the network obey the mass conservation equation, and the inlet pressure and outlet pressure are known, thus pressure at each node can be calculated by solving the following system of equations:

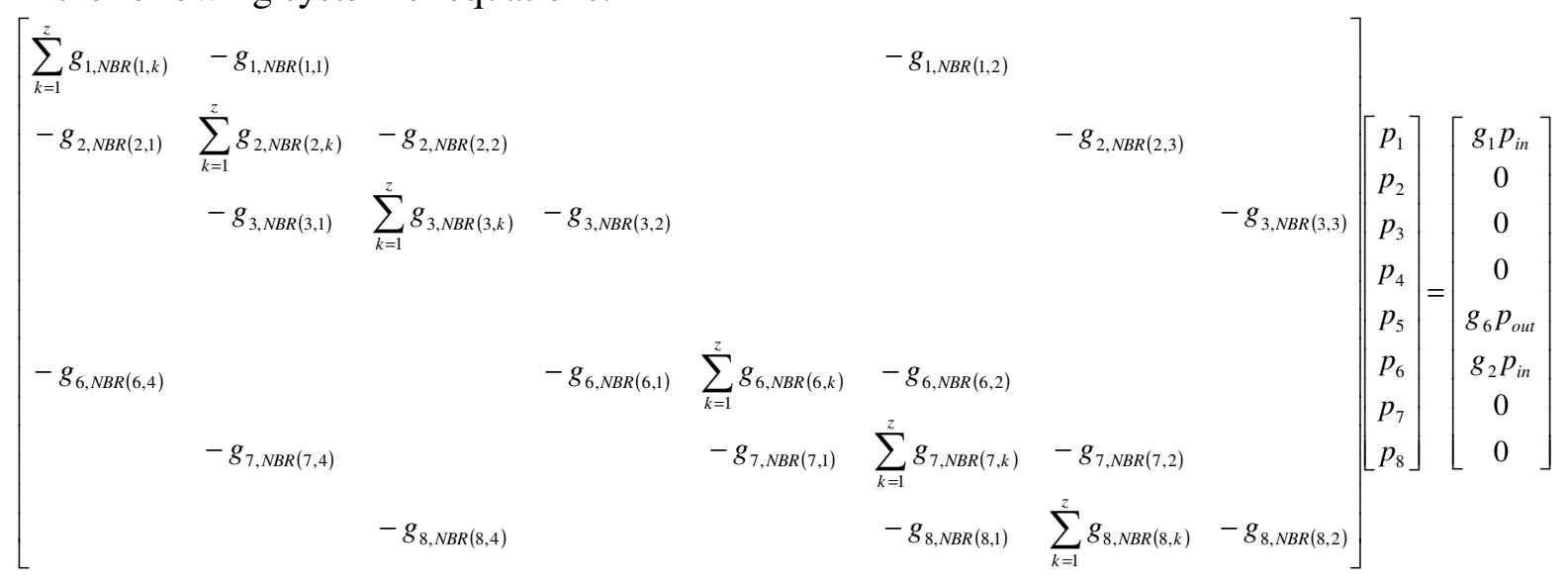

Consequently, flux through the network can be calculated using the equation:

$$
\mathrm{q}_{\text {total }}=\sum_{\mathrm{i}} \mathrm{g}_{\mathrm{i}} \times\left(\mathrm{P}_{\mathrm{in}}-\mathrm{P}_{1}\right)
$$

Permeability can be estimated from the Darcy's equation:

$$
\mathrm{q}=\frac{\mathrm{kA}}{\mu} \times \frac{\Delta \mathrm{P}}{\mathrm{L}}
$$




\subsection{PERMEAbILITY ESTIMATION OF DAMAGED Formations}

Permeability impairment is often used to characterize the level of formation damage. Therefore, permeability estimation is important for the understanding of the formation damage. The network model introduced in the previous section provides a feasible platform to estimate permeability of a damaged formation. However, the regular network model is most suitable for equal size sphere packing. Therefore, in this thesis, the regular network model is adjusted to process poorly sorted sphere packs, in particular, those contains two well-separated grain sizes. While Deluanay tessellation is widely used to produce pore throat networks of granular media, there is surprisingly little commentary on quality of the networks (i.e. how well tetrahedra represent the pores). Al-Raoush et al. deal with this issue [25], however only in monodisperse sphere packs. We have found no literature on applicability of Delaunay tessellation in packs with wide distribution of sphere sizes. We provide a method for packs with two different sizes in the following sections.

\subsubsection{Network Construction for Media with Two Well-Separated Grain Sizes}

Delaunay tessellation is an efficient way to obtain a network of pores and throats for well-sorted sphere packs. Delaunay tessellation, however, is a geometrical method and the resulting tetrahedral are functions of sphere centers only (and not the sphere sizes). Pore throats (tetrahedral sides) it finds in poorly-sorted sphere packs might not be physical constriction to the flow. In a damaged formation, invaded particles have much smaller size compared to the original formation grains, and these particles are often embedded in the void space of the formation matrix. Figure 31 shows the 2D Delaunay tessellation result of spheres with two well-separated sizes. The size difference between the big sphere and the small sphere is about five times or more, which is typical for the 
formation damage situation. Apparently, the triangle $\mathrm{ABC}$ from the Delaunay tessellation is not a physical representative of the pore body, and the edge $\mathrm{BC}$ is also not a physical flow path.

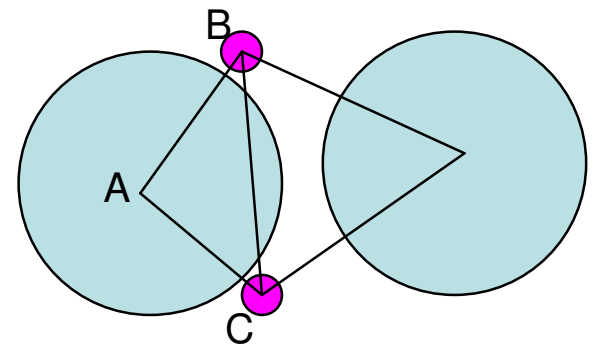

Figure 31. 2D Delaunay tessellation of spheres with two well-separated sizes

The invaded particles in the damaged formation often attach to the surfaces of formation grains as shown in the left of Figure 32. Because the three small particles are the closest ones to the big particle, according to the rules of 3D Delaunay tessellation, a tetrahedral is formed to represent the basic cell of the network model. Each face of the tetrahedral represents a pore throat. Clearly, the surface area of face $\mathrm{ABC}$ is rather small, and it is difficult to find the inscribed circle which can be used to estimate the pore throat size. In a real situation, it is not the physical flow path, either.
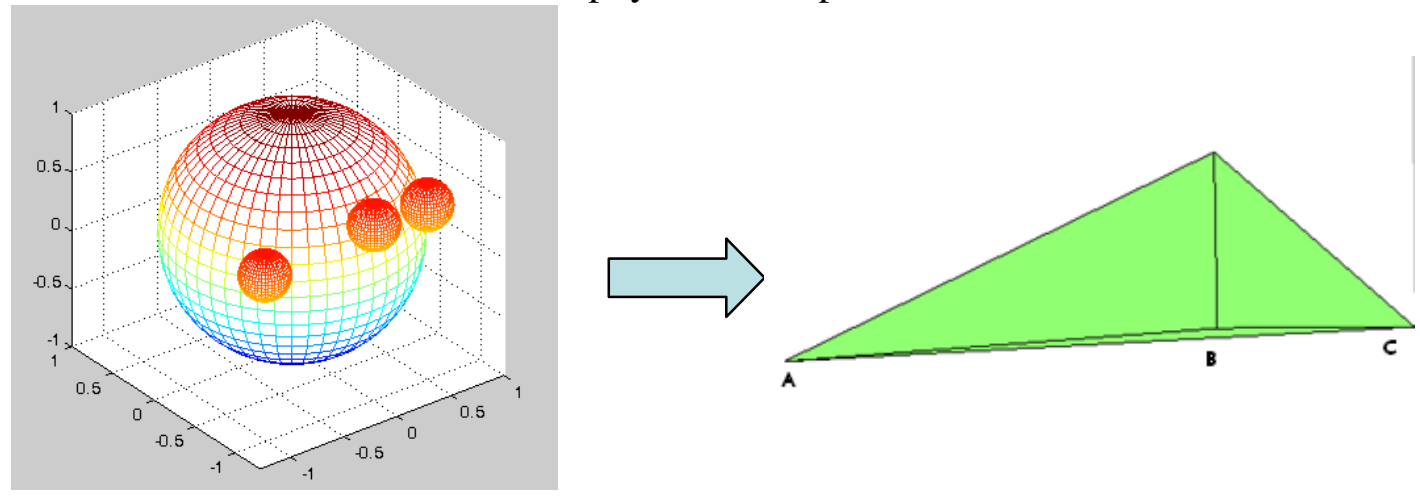

Figure 32. 3D Delaunay tessellation of spheres with two well-separated sizes

Besides the problem described above, another shortcoming for applying Delaunay tessellation to poorly sorted sphere packs is the computational demand. In the severely damaged formation or the formation very close to the well bore, the quantity of invaded 
particles is large. By considering all the particles in Delaunay tessellation, the resulting network contains a large volume of pores. Consequently the computational demands will be increased greatly.

The problem is two-fold. Even if Delaunay triangles are the basis for pores, the result is a large size network. In the example shown on the left in Figure 33, five big particles together with seventeen small particles generate more than thirty pores. In a similar situation in $3 \mathrm{D}$, the two separated sizes of particles will cause a much bigger mass balance equation matrix and significantly increases computational complexity. A further problem was discussed before: triangles whose vertices are from two different size classes, do not necessarily form meaningful pores.
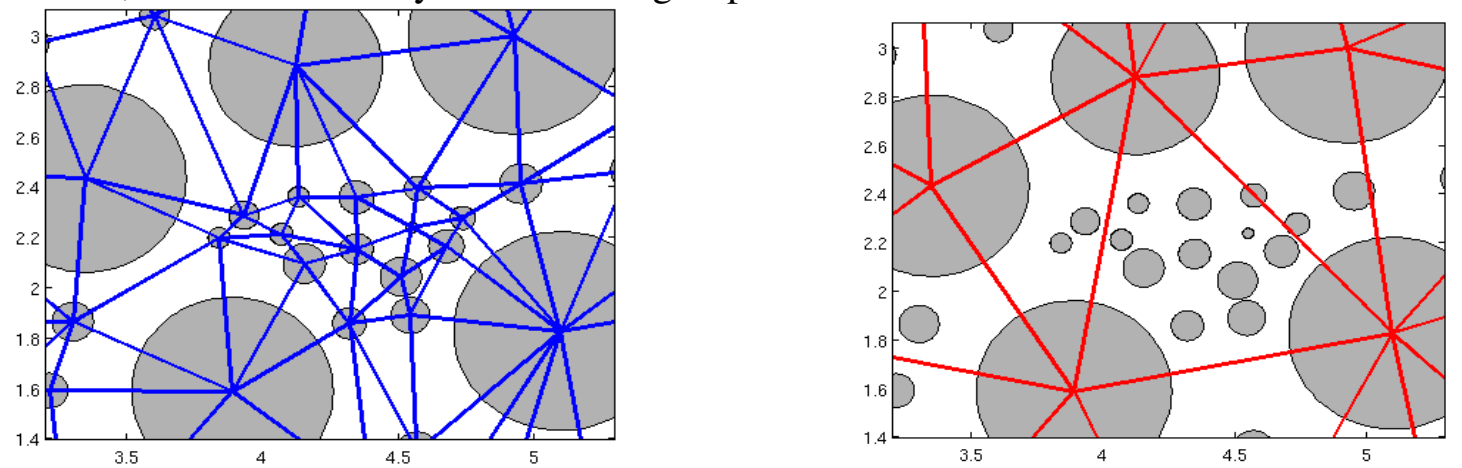

Figure 33. In the left diagram, a large number of triangles is created from the 2D Delaunay tessellation of the disk pack by considering all the particles. In the right diagram, much fewer triangles are created from the 2D Delaunay tessellation of the disk pack by considering just big particles.

Similar phenomena also occurs in 3D Delaunnay tesselation. In the example shown in Figure 34, four big particles together with a bunch of small particles result in number of tetrahedrons; while if just considering big particles for the tessellation, only one tetrahedron is generated (see Figure 35), which greatly reduces the computational complexity and demands in the further network model computation. 


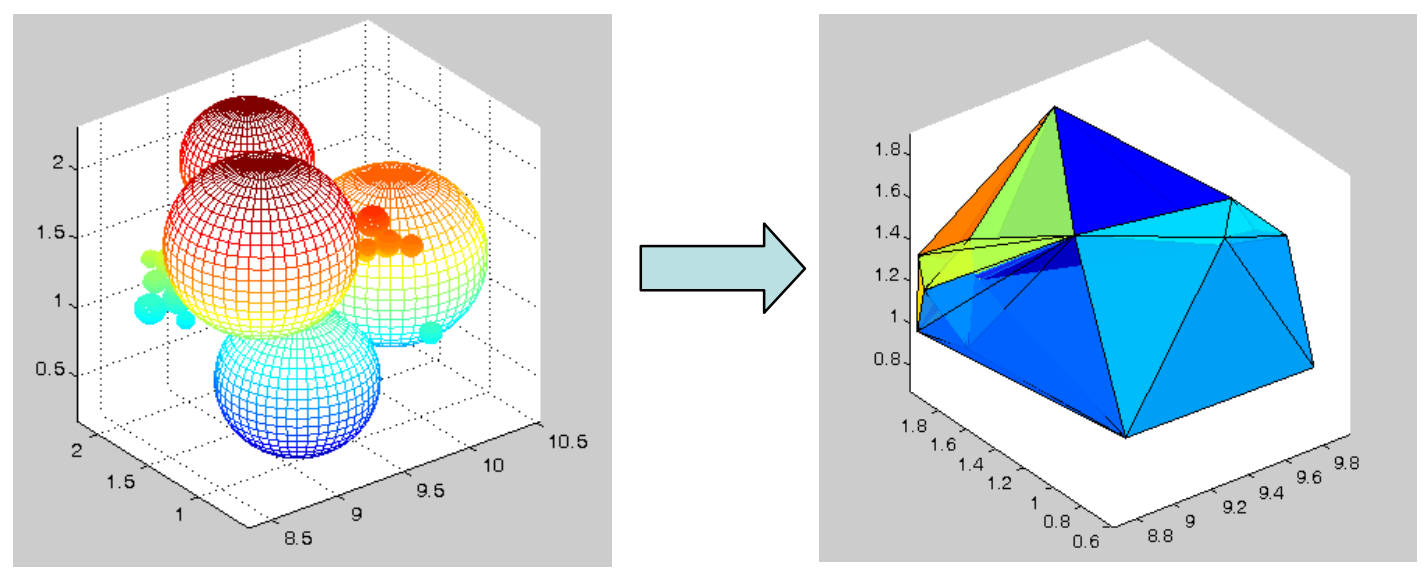

Figure 34. A large number of tetrahedral is created from the 3D Delaunay tessellation of the sphere pack by considering all the particles.
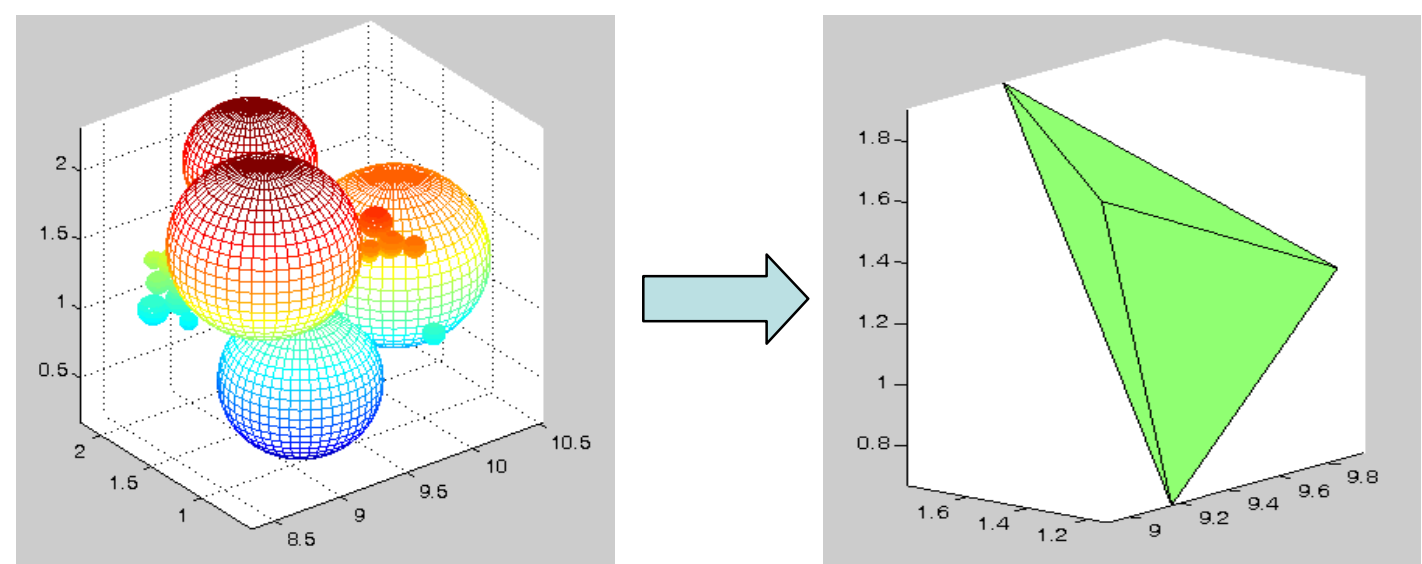

Figure 35. A smaller number of tetrahedra is created from the 3D Delaunay tessellation of the sphere pack by considering just big particles.

Examples above show that standard Delaunay tessellation is not applicable to the damage formation sample, which includes both formation grains and invaded particles. An updated Delaunay tessellation is proposed in this study for sphere packs containing the grains with two well separated sizes. In this updated method, big particles and small particles are considered separately. The network structure, pore throats and pore bodies are built according to the tessellation result from big particles, and small particles are taken into account as porosity and permeability reduction based on exact geometrical calculation. 


\subsubsection{Pore Throat Porosity Estimation}

The invaded particles are embedded in the original formation matrix, which is represented by a network of interconnected pore throats and pore bodies. These small particles occupy the void space of the pore throats and pore bodies, thus reduce their porosity. Because pore throats are the narrowest parts of the flow paths, reducing the porosity of pore throats results in permeability impairment for the damaged formation. Therefore, pore throats porosity estimation is vital for further permeability estimation.

In this thesis, a pore throat is considered as a cylindrical tube connecting two pore bodies. Invaded particles, represented by spheres, are deposited in the tubes as shown in Figure 36.

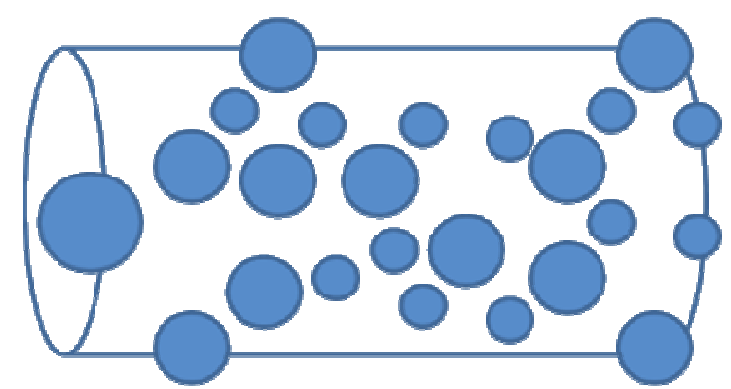

Figure 36. A schematic of invaded particles deposited in the pore throat

The porosity of the pore throat can be estimated by the following equation:

$$
\varphi=\frac{\mathrm{V}_{\text {tube }}-\mathrm{V}_{\text {solid }}}{\mathrm{V}_{\text {tube }}}
$$

where $\mathrm{V}_{\text {solid }}$ represents the total volume of solid particles inside the tube.

Relative position between particle centers and pore throat center lines, particle sizes and pore throat sizes are used to determine whether particles are totally inside the tube, partially inside or totally outside the tube. For the case of particles partially inside the tube, inside volume calculation is rather complicated, since the intersection between a sphere and a cylinder is a curved surface as shown in Figure 37. To simplify the problem, 
the sphere is cut by two planes: one intersects with the sphere at the lowest point of the intersection curve (see Figure 37) and another intersects with the sphere at the highest point. The average volume of the cut "crowns" is used to estimate the inside volume of the particle.

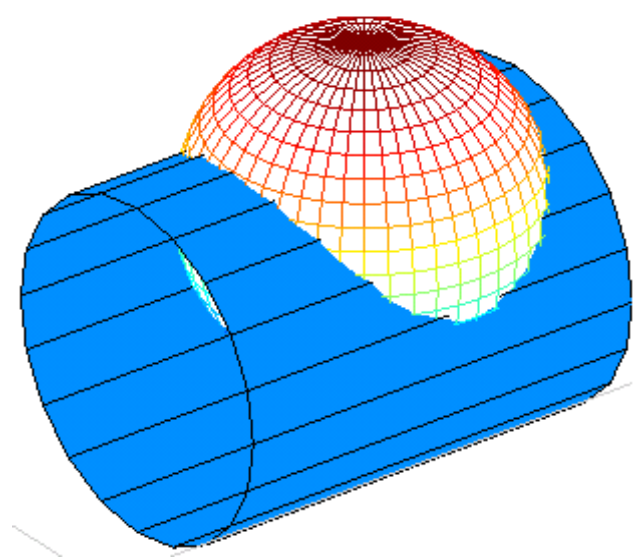

Figure 37. A particle is partially inside a pore throat. The intersection between a sphere and a cylinder is a curved surface.

\subsubsection{Pore Body Porosity Estimation}

As shown in Figure 38, invaded particles may also be deposited in pore bodies and cause porosity reduction of pore bodies. 


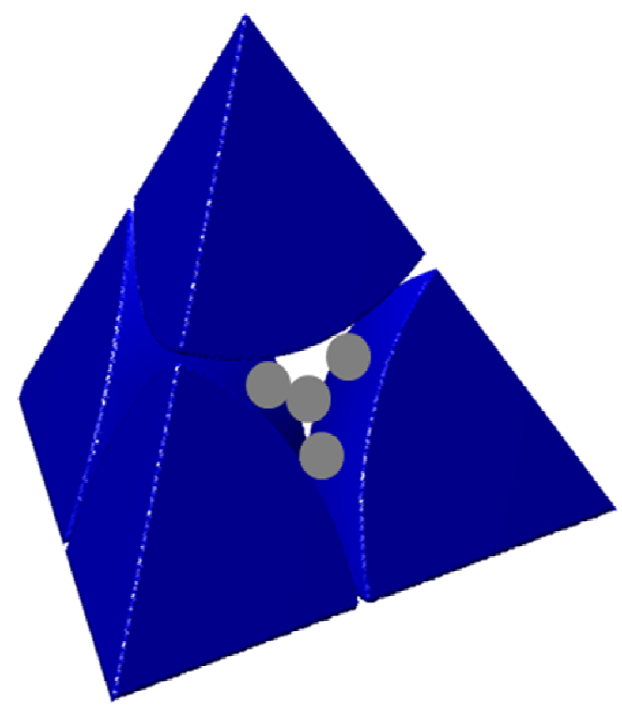

Figure 38. Invaded particles are deposited in a pore body

The porosity of pore bodies can be calculated using the same approach for the porosity of pore throats. The sides of tetrahedral delineating pore body are planes. Thus, for the case of a particle partially inside a pore body, precise geometry calculations are conducted to calculate the volume of inside particles.

\subsubsection{Pore Throat Conductance Calculation}

Pore throat conductance is one of the key inputs for the network permeability estimation. In the regular network model, a pore throat is considered as an empty cylindrical tube linking two pore bodies, and the conductance of the tube can be estimated by Hagen-Poiseuille equation:

$$
g=\frac{\pi r_{i}^{4}}{8 \mu L}
$$

In this study, invaded particles occupy the void space of the cylindrical tube, and reduce the porosity of the tube. Therefore, the conductance of pore throats needs to be reestimated by taking the invaded particles into account. Relative size and position between the invaded particles and the pore throat determine the pore throat conductance. When the 
radius of an invaded particle is larger than the radius of the pore throat, and the invaded particle sits across the cross section of the pore throat as shown in Figure 39, then the pore throat is blocked although the pore throat porosity might be still greater than zero.

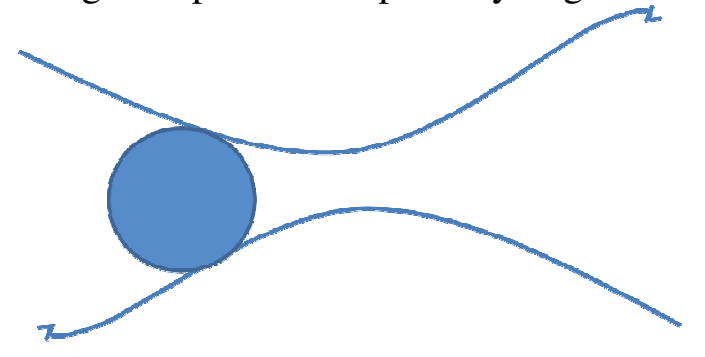

Figure 39. A pore throat is blocked by an invaded particle

Particles, smaller than the pore throats, are more likely deposited in the pore throats and bridge the pore throats. There are different types of deposition of small particles: they may only deposit on the surface of the formation grains, or may clog in the whole tube. Two different models of throat conductance estimation are proposed in this study.

\section{Surface deposition model}

The damaged formation specimens in this thesis are generated from the particle invasion simulation. It is assumed that each invaded particle is under the action of both fluid drag force and gravitational force. Therefore, the invaded particles tend to deposit on the surface of the formation grains where the supporting forces from the formation grains balance the gravitational force of the particle, and the interaction forces between the particle and formation grains balance the drag force exerted by the transporting fluid. Invaded particles, which are smaller than pore throats, penetrate into pore throats and line on the surface of pore throats under the action of gravitational forces and collision forces.

The deposited particles occupy the void space of pore throats, and thus cause the conductance reduction of pore throats. In this model, we assume that invaded particles 
are deposited along the surface and decrease the radius of pore throats (see Figure 40). Therefore, a smaller cylindrical tube is used to re-estimate the pore throat conductance. The radius of the smaller tube can be calculated using the following equation:

$$
\begin{aligned}
\pi \times \mathrm{r}_{\mathrm{e}}^{2} \times \mathrm{L} & =\pi \times \mathrm{r}_{\mathrm{i}}^{2} \times \mathrm{L} \times \varphi \\
\mathrm{r}_{\mathrm{e}} & =\mathrm{r}_{\mathrm{i}} \times \sqrt{\varphi}
\end{aligned}
$$

where $r_{e}$ is the equivalent radius of the pore throat;

$r_{i}$ is the initial radius of the pore throat;

$\mathrm{L}$ is the length of the pore throat;

$\varphi$ is the porosity of the pore throat.

The estimated equivalent radius can be used for the further calculation of the tube conductance by Hagen-Poiseuille equation:

$$
g=\frac{\pi r_{i}^{4} \varphi^{2}}{8 \mu L}
$$

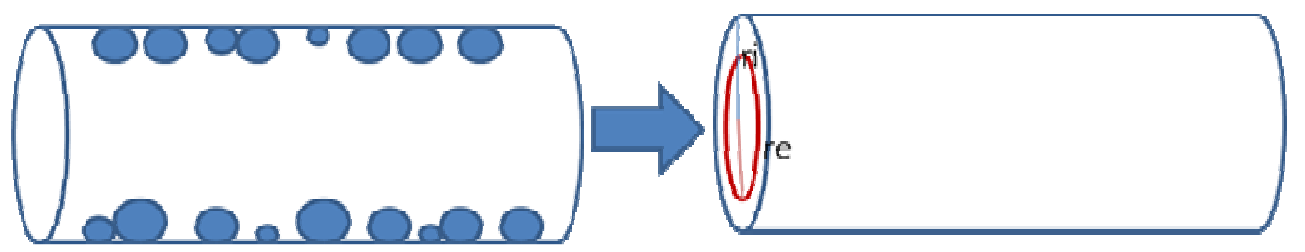

Figure 40. Surface deposition model

The surface deposition model is more applicable when the porosity of the tube is high.

\section{Clogging model}

After surface deposition, if more particles penetrate into and are deposited on a pore throat, then these particles will start to clog the pore throat, as shown in Figure 41. Consequently, the pore throat becomes another porous medium. There are various ways to estimate the permeability of the resultant porous medium. We can either use network model or use empirical Carman-Kozeny equation. Since the computational demands of network models are relative high if used for every throat, and Carman-Kozeny equation 
provides reasonable estimation of the permeability according to the particle size, CarmanKozeny equation [33] (shown below) is adopted in this study for the permeability estimation of clogged pore throats.

$$
\mathrm{k}=\frac{\varphi^{3} \mathrm{D}_{\mathrm{p}}^{2}}{72 \tau(1-\varphi)^{2}}
$$

where $\mathrm{k}$ is the estimated permeability of the pore throat;

$\mathrm{D}_{\mathrm{p}}$ is the particle diameter;

$\tau$ is the tuortuosity;

$\varphi$ is the porosity of the pore throat.
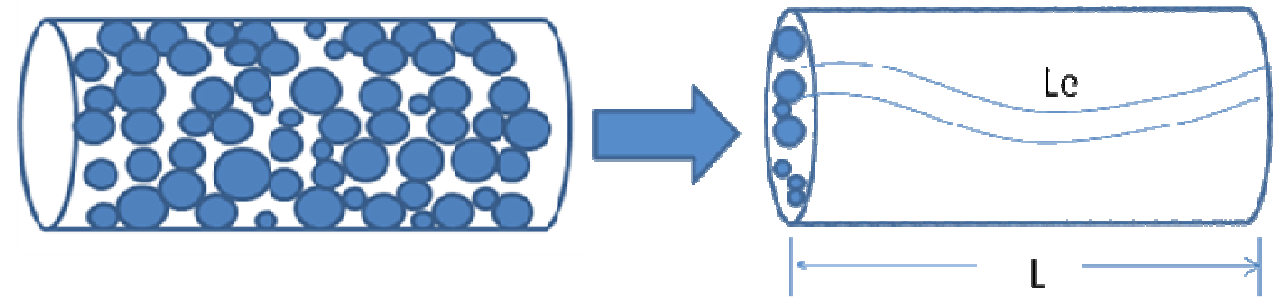

Figure 41. Clogging model

Because the conductance of pore throats instead of the permeability is needed for further permeability estimation of the whole network, calculate pore throat permeability is converted to the pore throat conductance via the following equation:

$$
\mathrm{g}=\frac{\mathrm{kA}}{\mu \mathrm{L}}
$$

Substituting permeability $\mathrm{k}$ using permeability estimation equation in the above equation, we can get:

$$
g=\frac{1}{72 \tau} \frac{\varphi^{3} D_{p}^{2}}{(1-\varphi)^{2}} \frac{A}{\mu L}
$$

where $\mathrm{k}$ is the estimated permeability of the pore throat;

$\mathrm{A}$ is the cross section area of the pore throat;

$\mu$ is the viscosity of the fluid;

$\mathrm{L}$ is the length of the pore throat. 
It is more reasonable to apply this model in the situation when the porosity is relative low.

These two models are applicable to different deposition situations. Pore throat porosity is one of the key factors which can be used to characterize the deposition situation. Because the porosity of a disordered equal sphere pack is $36 \%$, it is reasonable to conclude that the clogging model should be used when the pore throat porosity is lower than $36 \%$. When the pore throat porosity is higher than $80 \%$, it is more reasonable to apply the surface deposition model. When the pore throat porosity is in the range of $36 \%-80 \%$, more detail analysis about the relative position between deposited particles and pore throats should be done to decide which model should be applied.

\subsection{RESULTS AND DISCUSSION}

The updated network model introduced in the previous sections of this chapter is used to estimate the permeability of the damaged formation specimens, which are generated from $\mathrm{PFC}^{3 \mathrm{D}}$ simulation. In Chapter 2, the effects of initial porosities and particle sizes on porosity reduction and invasion depth have been studied in detail. The effects of these factors on the permeability impairment are studied in this chapter.

\subsubsection{Permeability Impairment Study of Specimen 1}

The related properties of specimen 1 are shown in Table 7 . 
Table 7. Properties of Specimen 1

\begin{tabular}{|c|c|}
\hline Properties & Value \\
\hline Specimen Size & $8 * 4 * 4$ \\
Length $(\mathrm{mm}) *$ Width $(\mathrm{mm}) *$ Height $(\mathrm{mm})$ & 0.25 \\
\hline Formation Grain Radius $(\mathrm{mm})$ & $0.07-0.08$ \\
\hline Particle Size (mm) & $30 \%$ \\
\hline Initial Porosity & $28 \%$ \\
\hline Damaged Porosity & 4 \\
\hline Invaded Depth (mm) & \\
\hline
\end{tabular}

According to the study conducted in Chapter 2, for this specimen, the amount of invaded particles is relative small and most of them are located in the region which is 0$1 \mathrm{~mm}$ away from the wellbore. Consequently, we can speculate that different regions of the specimen have different permeability impairment. Thus, the specimen is cut into different regions according to their distance to the formation face; and permeability impairment is examined for these regions separately, as shown in Table 8.

Table 8. Permeability Impairment of Specimen 1

\begin{tabular}{|c|c|}
\hline $\begin{array}{c}\text { Distance to Formation Surface } \\
(\mathbf{m m})\end{array}$ & $\begin{array}{c}\text { Permeability Reduction } \\
\text { Percentage }\end{array}$ \\
\hline $0-1$ & $25 \%$ \\
\hline $1-2$ & $3.8 \%$ \\
\hline $2-3$ & $3.3 \%$ \\
\hline $3-4$ & $3.7 \%$ \\
\hline $4-5$ & $0 \%$ \\
\hline $5-6$ & $0 \%$ \\
\hline $6-7$ & $0 \%$ \\
\hline $7-8$ & $0 \%$ \\
\hline
\end{tabular}


Clearly, permeability reduces most in the first section; therefore, detail analysis is performed for this section.

A network of pores and pore throats are built from this section using the approach introduced in the previous sections. Pore structure and pore throat sizes are vital to the permeability of a porous medium. Therefore, they are examined first. Figure 42 shows the probability density plot of pore throat sizes and pore body sizes of this porous medium. As we can see from the figure, around $70 \%$ of pore body sizes are in the range of $0.05-0.1 \mathrm{~mm}$, and less than $6 \%$ is smaller than $0.05 \mathrm{~mm}$. However, for pore throats, around $30 \%$ is in the range of $0-0.05 \mathrm{~mm}$, which demonstrates that pore throats are the resistance to the fluid flow and control permeability of the porous medium.

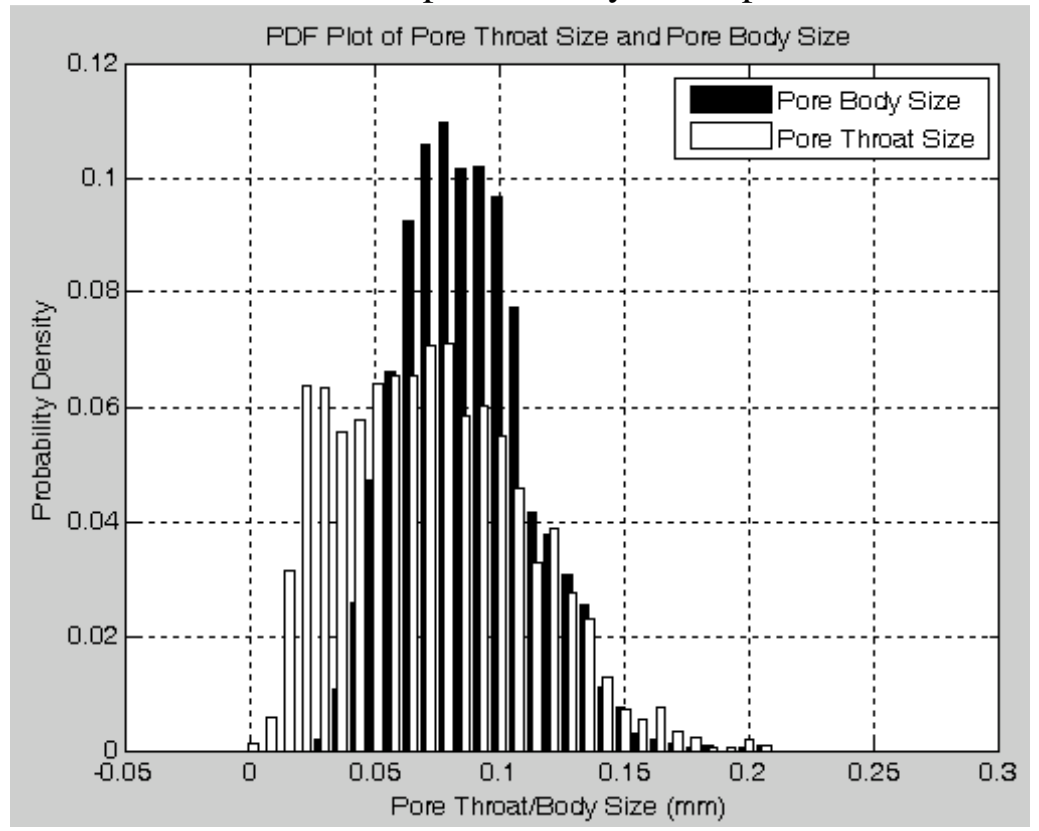

Figure 42. PDF plot of pore throat size (radius) and pore body size (radius). The plot is generated from Specimen 1.

Close examination of the plot shows that some pore throats have size zero. In this study, pore throat size is defined as the radius of the inscribed sphere which is touching three grains forming the pore throat. As shown in Figure 43, when three grains are 
overlapping each other, there is no void space for the fluid to flow through; and it is impossible to find an inscribed sphere. Therefore, the pore throat size becomes zero.
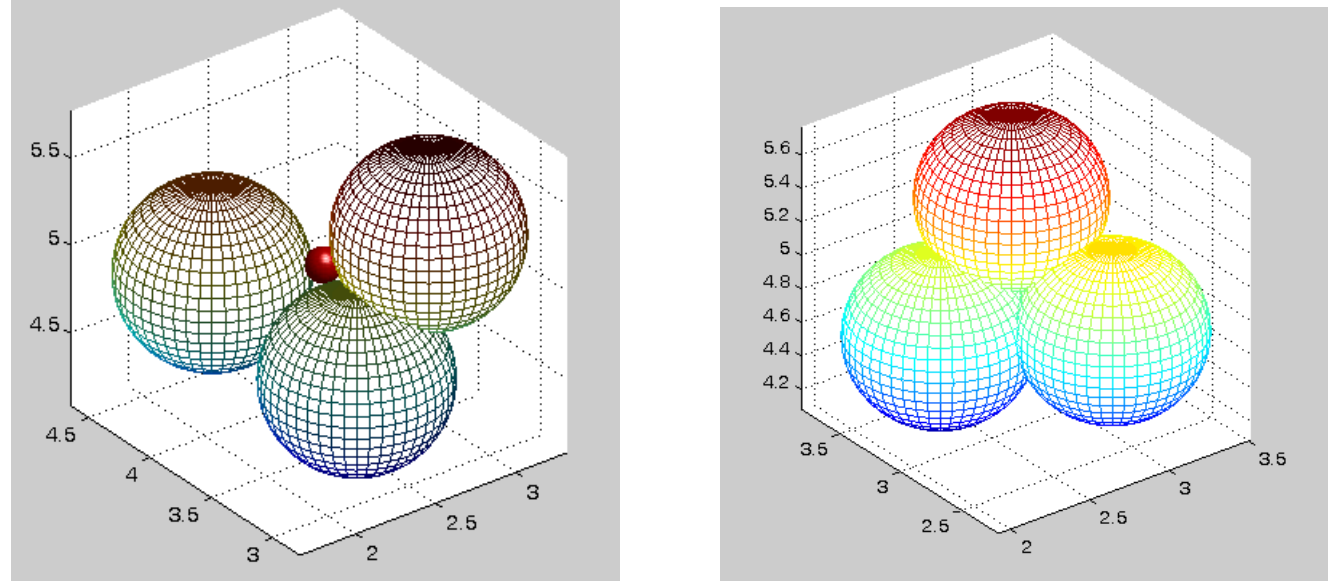

Figure 43. Pore throat radius is the radius of a sphere inscribed among the spheres defining the throat. In the left figure, three grains have some overlap, but there is still void space among them, thus an inscribed sphere can be found. In the right figure, three grains overlapped each other heavily with, no void space left among them, and thus it is impossible to find an inscribed sphere.

The overlapping between grains is caused by dense, slightly overlapping pack as required by DEM (see Chapter 2). It is known that the porosity of a pack with disordered equal spheres is about 36\%. The specimen generated from PFC3D has the porosity around $30 \%$, thus overlapping is inevitable.

Figure 44 shows the probability density distribution of the pore throat length. About $80 \%$ throats have length in the range of $0.2-0.6 \mathrm{~mm}$.

Relative size and position between invading particles and pore throats determine the number of throats which will be blocked. The size of invading particles is in the range of $0.14-0.16 \mathrm{~mm}$, and about half of the pore throats have sizes smaller than $0.15 \mathrm{~mm}$. Therefore, the pore throat blocking is likely to happen. The results show that only a small amount of pore throats are blocked (see Figure 45). 


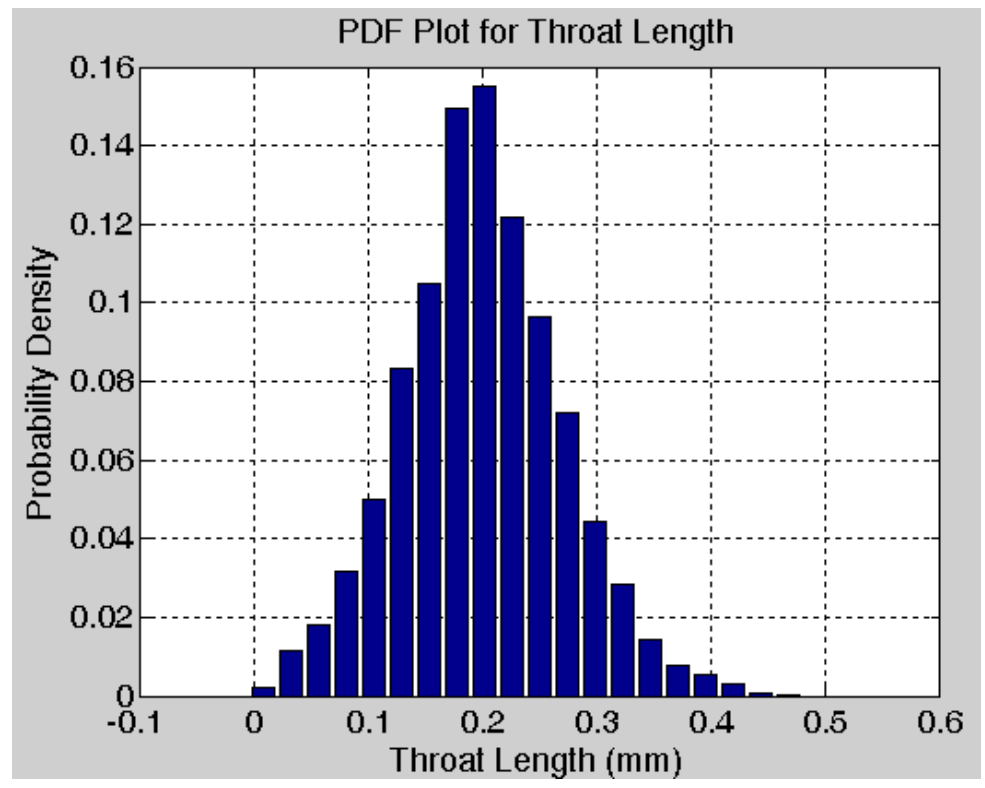

Figure 44. Pore throat length PDF plot of Specimen 1.

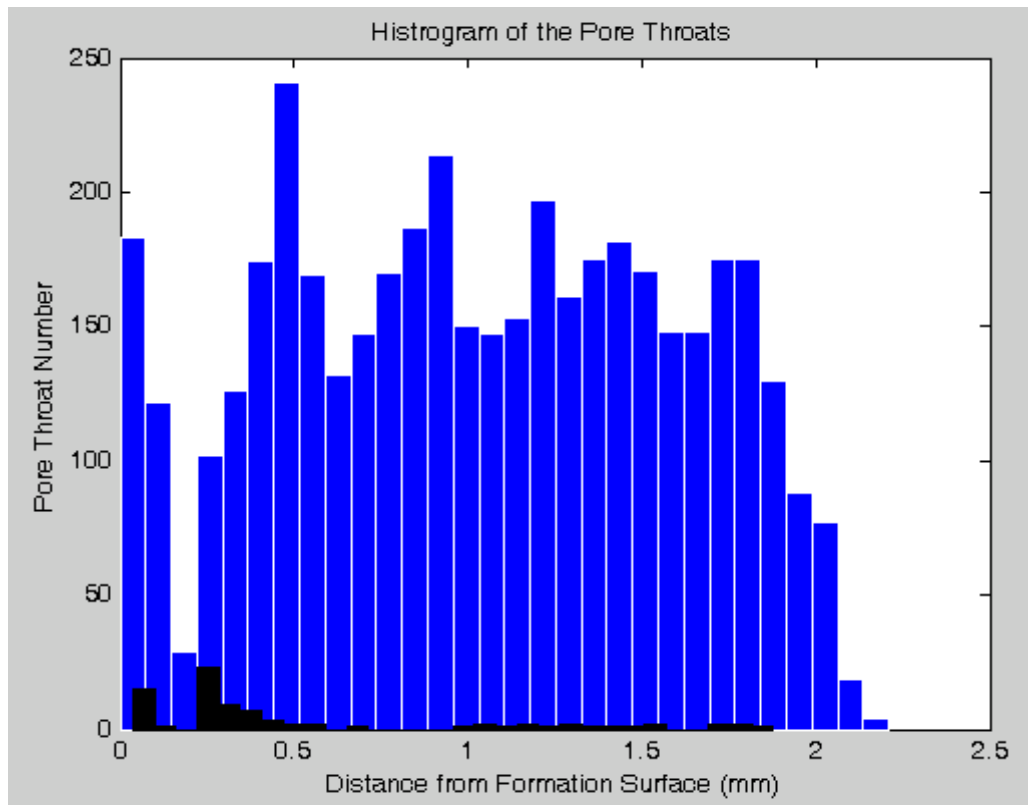

Figure 45. Histogram of all the pore throats and blocked pore throats for Specimen 1 along the distance from the formation face.

From the figure, it can be seen that most of the pore throat blocking happens close to the entrance of the porous medium. Compared to the original pore throat number, the 
blocked pore throat number is rather small. Therefore, the pore throat blocking of this specimen will not reduce the permeability greatly.

If the invaded particle size is bigger than the pore throat size, the pore throat is blocked; otherwise, the invaded particle is deposited or entrained in the pore throat and reduce the void space within the throat. Figure 46 shows the probability density plot of pore throat porosity. More than $80 \%$ of pore throats are not invaded by small particles. For those which contain small particles, the mean porosity is about $80 \%$. We conclude that the deposited particles do not greatly change the pore throat void space. The zero porosity in the plot represents the blocked pore throats.

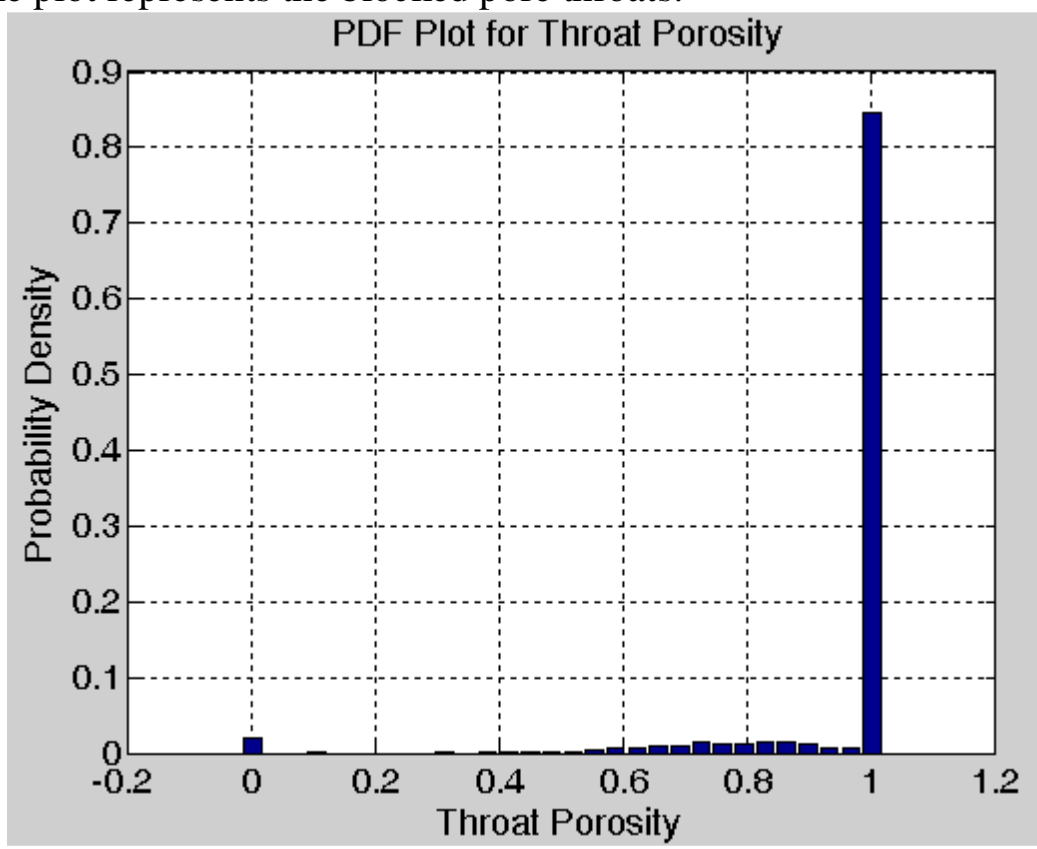

Figure 46. PDF plot of pore throat porosity. This plot is generated from Specimen 1. 


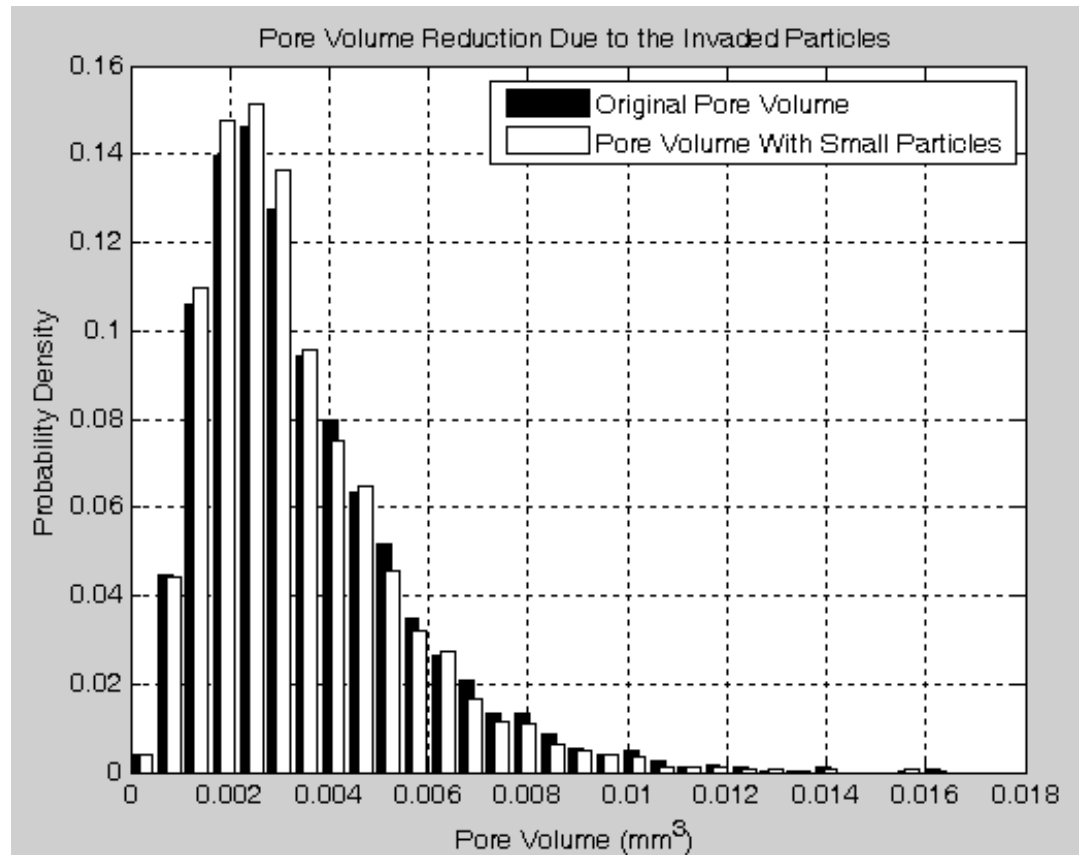

Figure 47. PDF plot of original pore volume and the pore volume with small particles deposited in the pore bodies. This plot is generated from Specimen 1.

The invaded particles also possibly deposit in the pore bodies. The reduction of the pore body volume due to invaded particles is shown in Figure 47. From the figure, it can be seen that the pore body volume is slightly reduced due to deposited small particles, and most reduction happens in the bigger pores, since particles tend to occupy void space in big pores other than that of small pores. Although invaded particles reduce the volume of pore bodies, the reduction is relatively low compared to that of pore throats. Therefore, it is still reasonable to assume the same pore throats are the narrowest part of the fluid flow path in the original and damaged sample.

Both surface deposition model and clogging model are used to re-estimate the conductance of pore throats, but they are applied in different situations. When the throat porosity is higher than $40 \%$, the surface deposition model is applied, otherwise the clogging mode is applied. Figure 48 shows the PDF plot of damaged conductance and original conductance. The difference between the original conductance and the damaged 
conductance is very small. Compared to the original formation, the damaged formation has more percentage of conductance with value of zero, which is caused by the pore throat blocking.

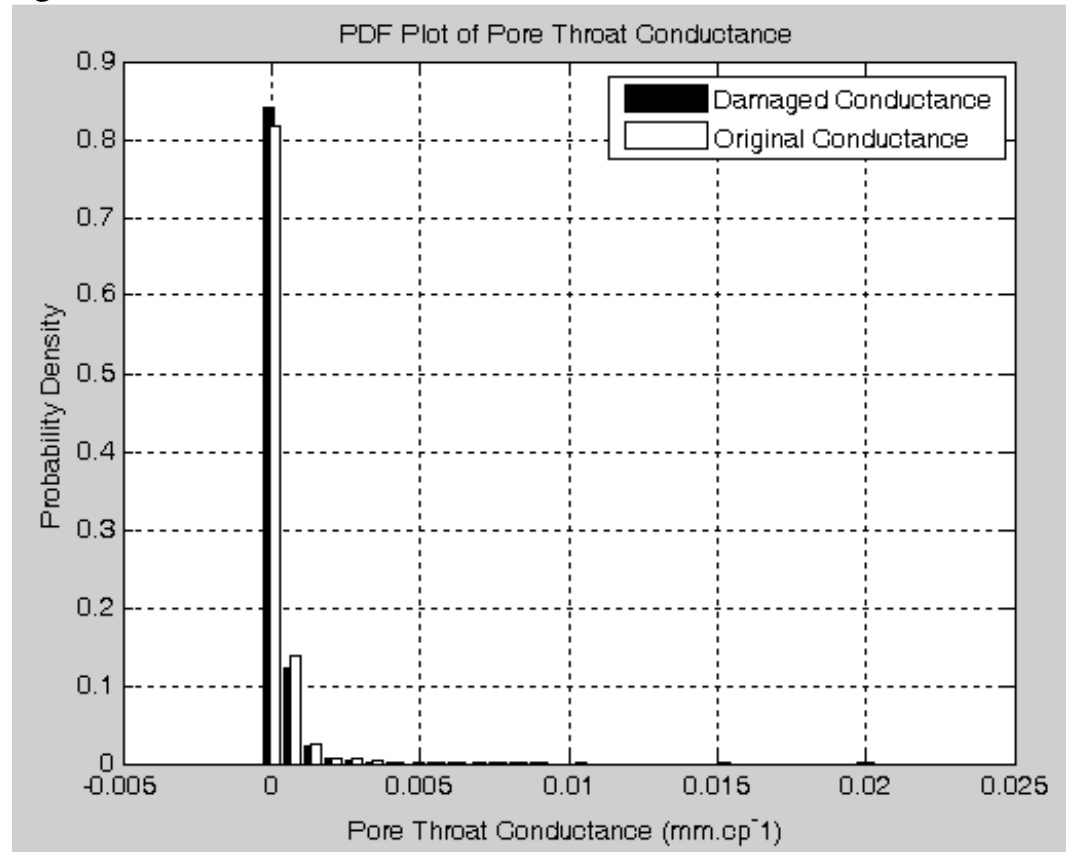

Figure 48. PDF plot of original conductance and reduced conductance. This plot is generated from Specimen 1.

Other sections of specimen 1 are also examined using similar methods as the first section. Since most invaded particles are deposited in the first section, permeability is reduced most in the first section. Figure 49 shows the normalized permeability impairment along the distance from the formation face. As expected, the permeability reduction of this specimen is very small, and is concentrated in the entrance of the formation matrix. 


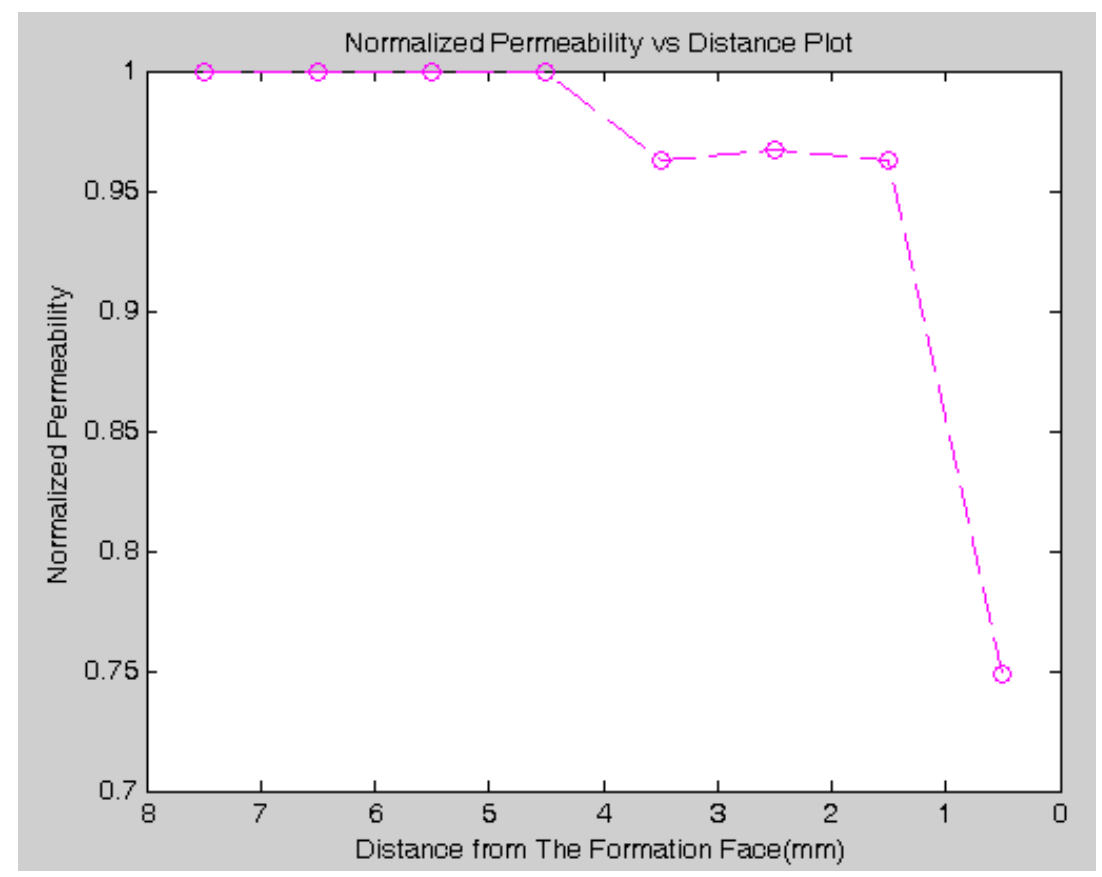

Figure 49. Normalized formation permeability along the distance from the formation face. This plot is generated from Specimen 1.

\subsubsection{Permeability Impairment Study of Specimen 5}

The related properties of specimen 5 are shown in Table 9.

Table 9. Properties of Specimen 5

\begin{tabular}{|c|c|}
\hline Properties & Value \\
\hline Specimen Size & $8 * 4 * 4$ \\
Length(mm)*Width(mm)*Height(mm) & 0.25 \\
\hline Formation Grain Radius(mm) & $0.05-0.06$ \\
\hline Particle Size $(\mathrm{mm})$ & $40 \%$ \\
\hline Initial Porosity & $32 \%$ \\
\hline Damaged Porosity & 8 \\
\hline Invaded Depth $(\mathrm{mm})$ & \\
\hline
\end{tabular}


Similar as previous specimens, the specimen is segmented into several sections according to the distance from the formation face. Table 10 lists the estimated permeability reduction value of different sections.

Table 10. Permeability Impairment of Specimen 5

\begin{tabular}{|c|c|}
\hline $\begin{array}{c}\text { Distance to Formation Surface } \\
(\mathbf{m m})\end{array}$ & $\begin{array}{c}\text { Permeability Reduction } \\
\text { Percentage }\end{array}$ \\
\hline $0-1$ & $32 \%$ \\
\hline $1-2$ & $4.7 \%$ \\
\hline $2-3$ & $7.8 \%$ \\
\hline $3-4$ & $5.7 \%$ \\
\hline $4-5$ & $0.6 \%$ \\
\hline $5-6$ & $0.3 \%$ \\
\hline $6-7$ & $0 \%$ \\
\hline $7-8$ & $0 \%$ \\
\hline
\end{tabular}

First section, which is $0-1 \mathrm{~mm}$ away from the formation face, contains most of the invaded particles. Therefore, permeability impairment of this section is examined in detail.

The pore size and the pore structure of this porous media are studied first. Figure 50 shows the probability density plot of the pore throat size and the pore body size. Compared to Specimen 1, which has initial porosity of 30\%, Specimen 5 has bigger pore throat sizes. Around $20 \%$ of the pore throats are smaller than $0.05 \mathrm{~mm}$, which is much lower than that of Specimen 1. For Specimen 1, about 30\% pore throats have size smaller than $0.05 \mathrm{~mm}$. Furthermore, due to the relative high porosity, severe overlapping is less likely to happen; therefore, there are no pore throats that have zero size. Moreover, pore body sizes of this specimen are also larger than those of Specimen 1. For Specimen 1, 
around $70 \%$ of pore body sizes are in the range of $0.05-0.1 \mathrm{~mm}$, while for this specimen, about $70 \%$ pore body sizes are in the range of $0.075-0.125 \mathrm{~mm}$.

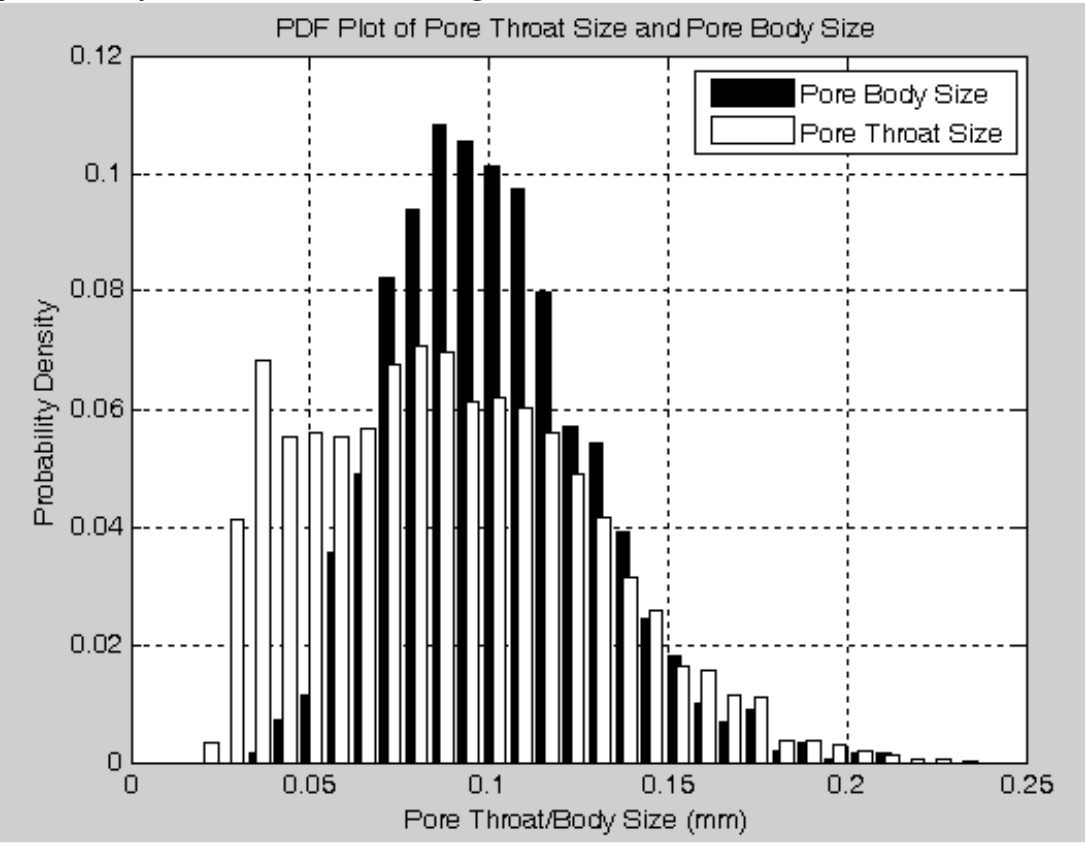

Figure 50. PDF plot of the pore throat size and the pore body size for Specimen 5.

Figure 51 shows the PDF plot of the pore throat length. It can be seen from the plot that the pore throat length of this specimen is very similar to that of Specimen 1. 


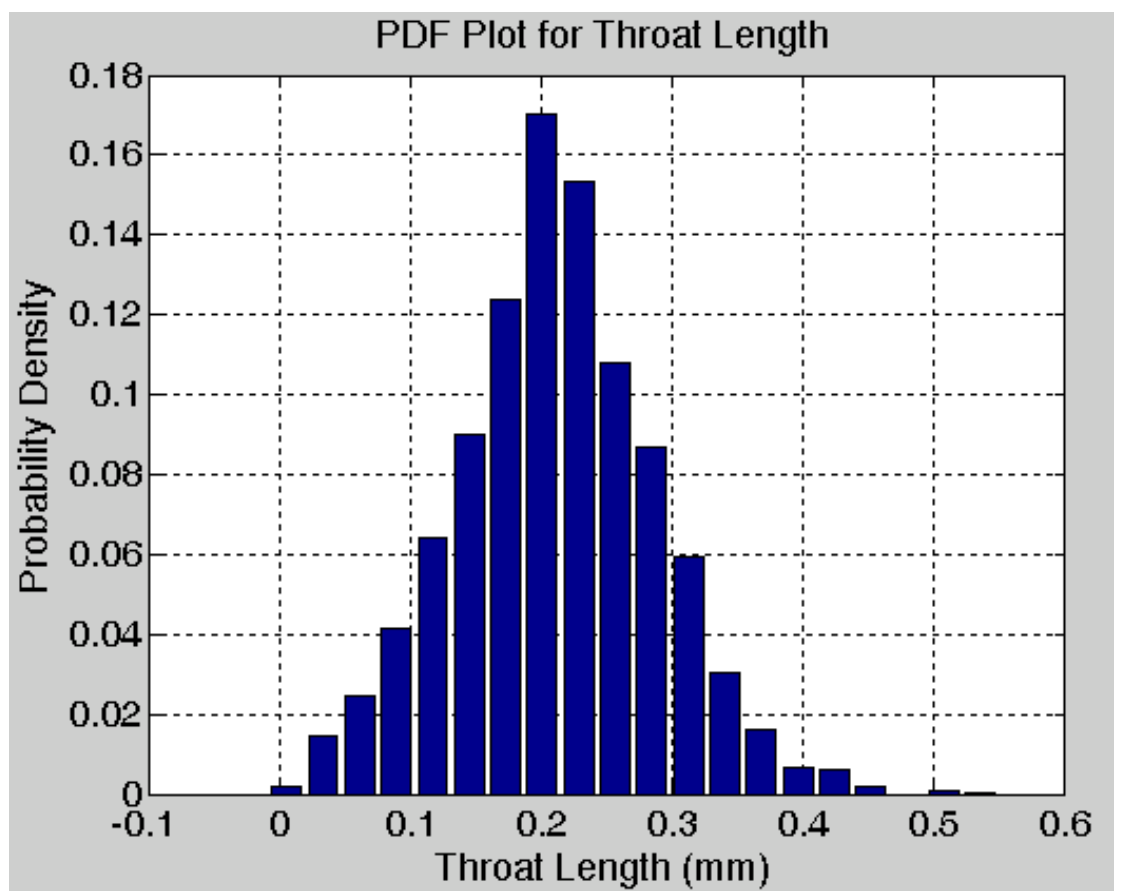

Figure 51. PDF plot of pore throat length. The plot is generated from specimen 5.

From the analysis, pore sizes are bigger than in Specimen 1 due to a higher porosity, further in this specimen invading, particle size is five times smaller than the formation grains. Therefore, more formation damage is expected. Simulation results show that more than 2000 small particles invaded into the porous medium, compared to around 600 invaded particles for Specimen 1. Pore throat blocking due to the particles invasion is possible because around $20 \%$ of pore throats have sizes smaller than invaded particle size. Figure 52 shows the histogram of the total pore throat number and blocked throat number along the distance from the formation face. About $3 \%$ of pore throats are blocked over a wide range of locations. Compared to the original number of pore throats at each location, the number of blocked pores can be ignored; moreover, the size of blocked pore throats is small, thus the permeability of the porous medium is not reduced greatly. 


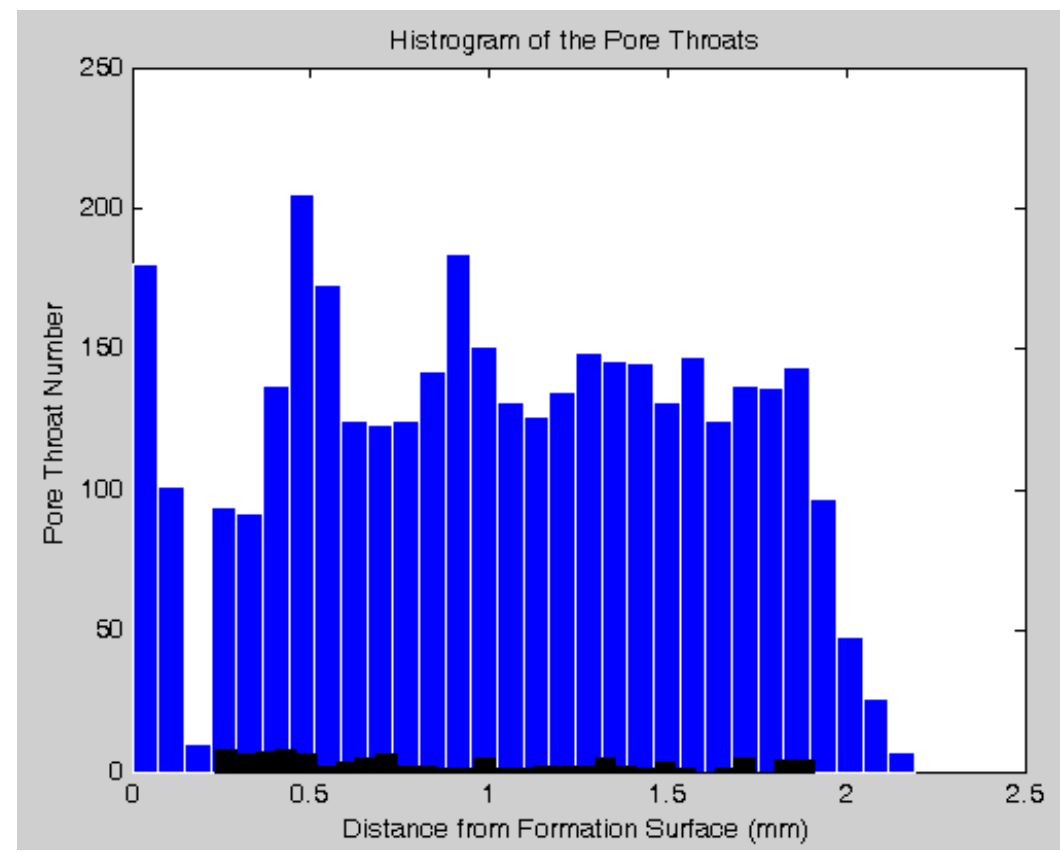

Figure 52. Histogram of all the pore throats and blocked pore throats along the distance from the formation face for Specimen 5.

Due to more particles invasion, the pore throat porosity is lower compared to that of Specimen 1. About more than half of the pore throats are invaded by particles, and most of the pore throats which contain particles have porosities higher than $60 \%$. The deposition situation should be further studied to apply for the proper models to estimate the conductance. 


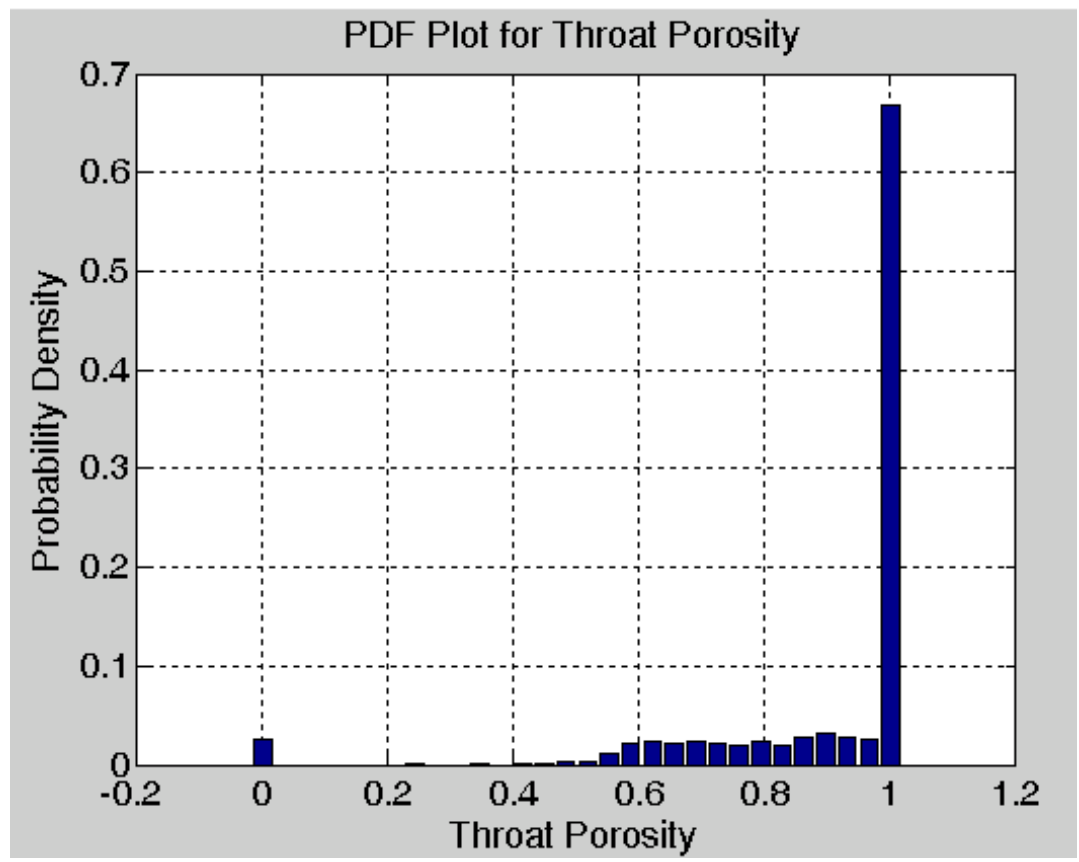

Figure 53. PDF plot of the pore throat porosity from Specimen 5.

The pore body volume reduction of this specimen is also higher than that of

Specimen 1. The figure also shows that more volume reduction happens in big pores.

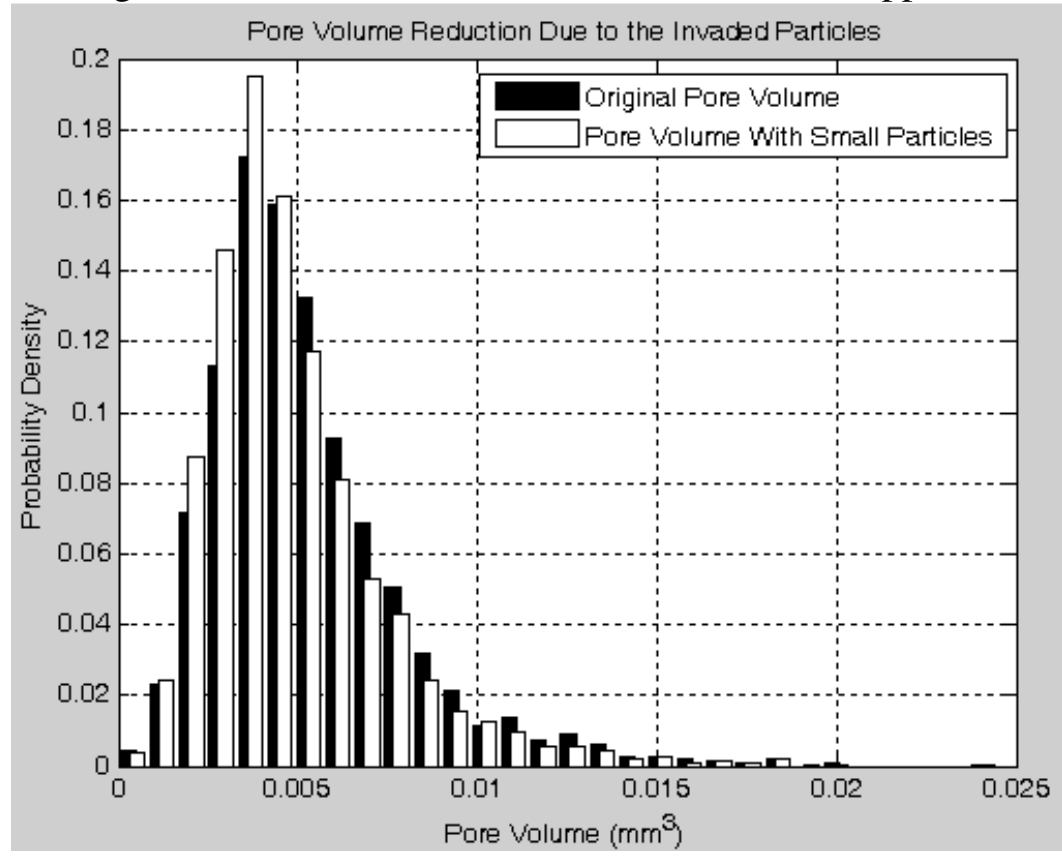

Figure 54. PDF plot of pore volume reduction due to invaded particles for Specimen 5. 
We further examine whether particles are more likely deposited in the pore throats than the pore bodies by comparing the solid volume of invaded particles inside pore bodies with that inside pore throats. For this section, the total volume of invaded particles is $11.57 \mathrm{~mm}^{3}$, and around $7.07 \mathrm{~mm}^{3}$ of particles are deposited in the pore throats compared to $4.5 \mathrm{~mm}^{3}$ in the pore bodies. The overall throat porosity in the studied section is about $80 \%$, and the porosity of pore body is $87 \%$. Therefore, we conclude that particles are somewhat more likely deposited in the pore throats.

Figure 55 shows the PDF plot of the original throat conductance and the damaged throat conductance. Compared to Specimen 1, a higher pore throat conductance decrease is observed in this specimen.

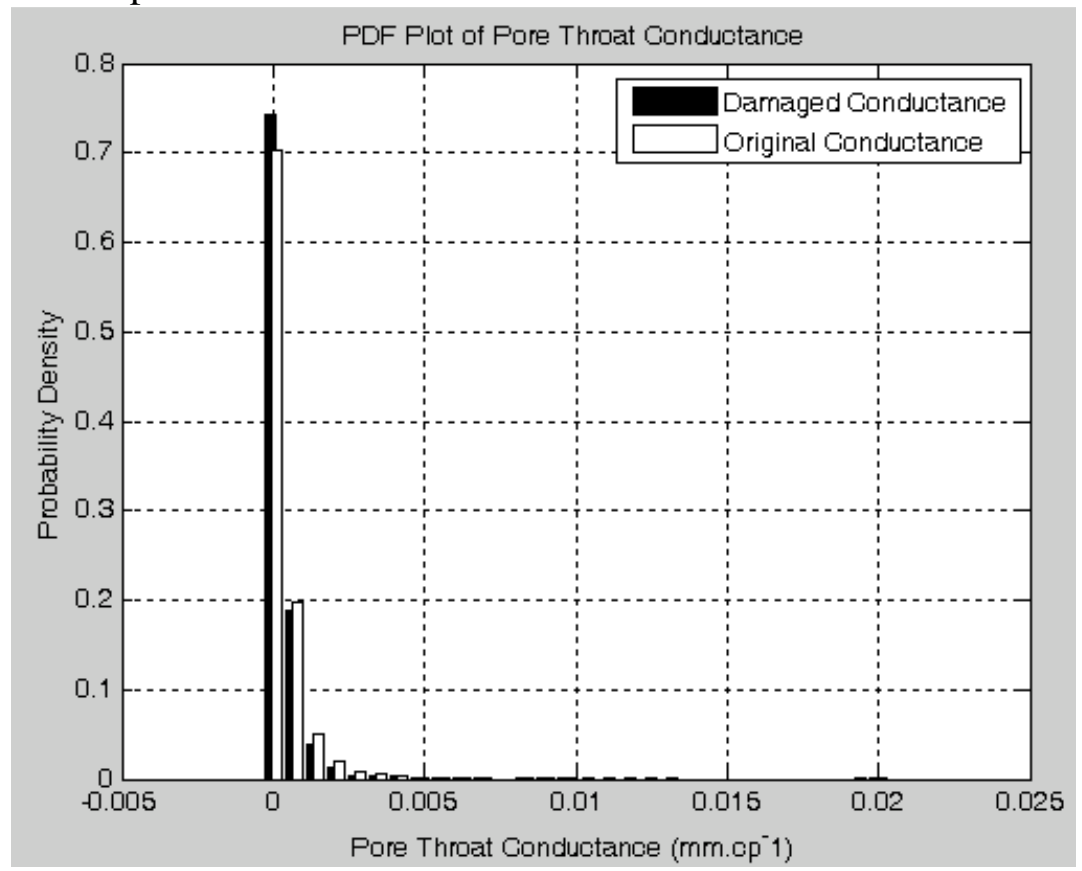

Figure 55. PDF plot of pore throat conductance for Specimen 5.

There are two models proposed in this thesis to estimate the conductance of pore throats containing small particles. Model selection is based on the deposition situation. Therefore, deposition situations for different porosity ranges are investigated in Figure 56 
and Figure 57. These figures show the normalized distance between the particle centers and the center line of pore throats. Each distance is normalized by the relevant throat radius. To get the statistical results of the deposition situation, all the pore throats within the same porosity range are plotted on the same figure. Figure 56 shows the statistical deposition situation of pore throats whose porosities are lower than $40 \%$. From the figure, almost half of particles deposit close to the center of the pore throats. This indicates that pore throats are clogged by invaded particles. Therefore, clogging model is selected to estimate the conductance of the pore throats with porosity lower than $40 \%$.

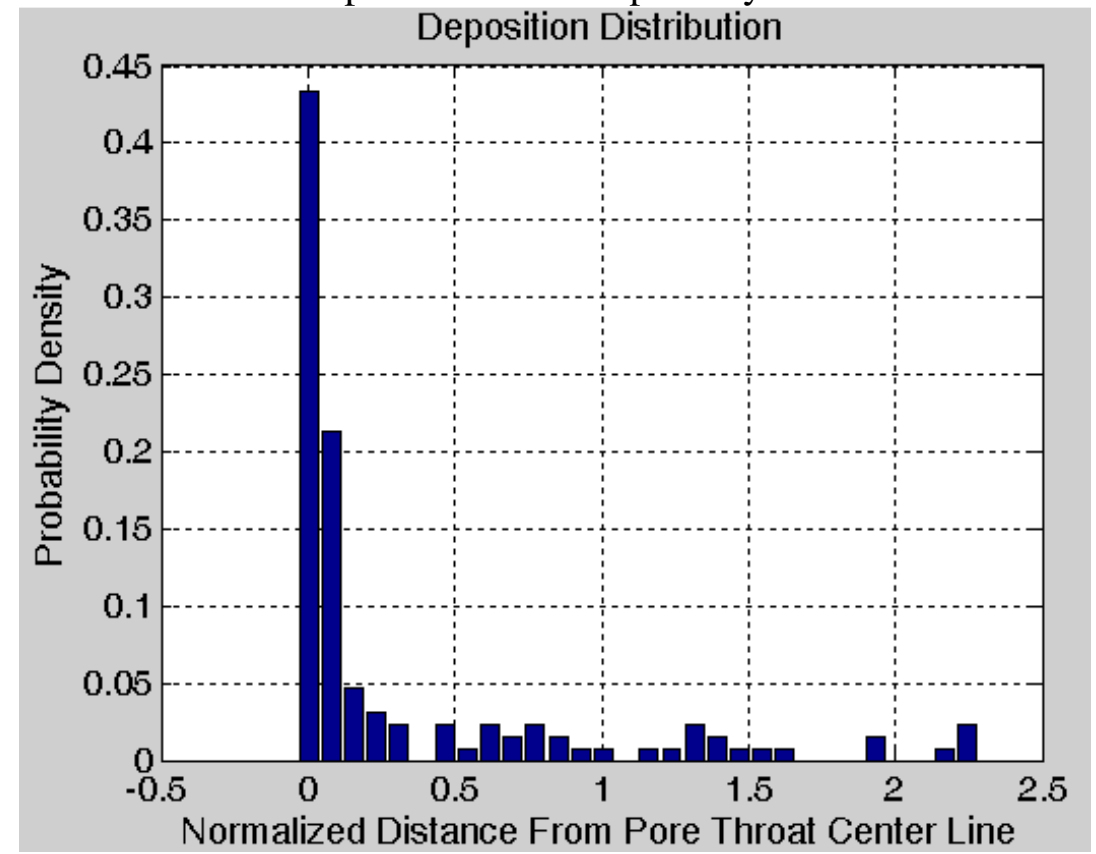

Figure 56. Deposition distibution of pore throats in Specimen 5 with porosity lower than $40 \%$.

Figure 57 shows the deposition distribution of pore throats with porosity range of $40 \%-60 \%$. Compared to the deposition situation in the previous porosity range, more particles (greater than $80 \%$ of total deposited particles) are deposited closer to the surface than to the center line of the pore throats, which indicates the majority of the deposited particles is lining the throat surface. Therefore, surface deposition model is applied in this 
case. A note of caution: the radii of deposited particles are not taken into account, so some of them may clog the throats although the centers of the particles are closer to the throat surface. More comprehensive analysis should be done in the future study.

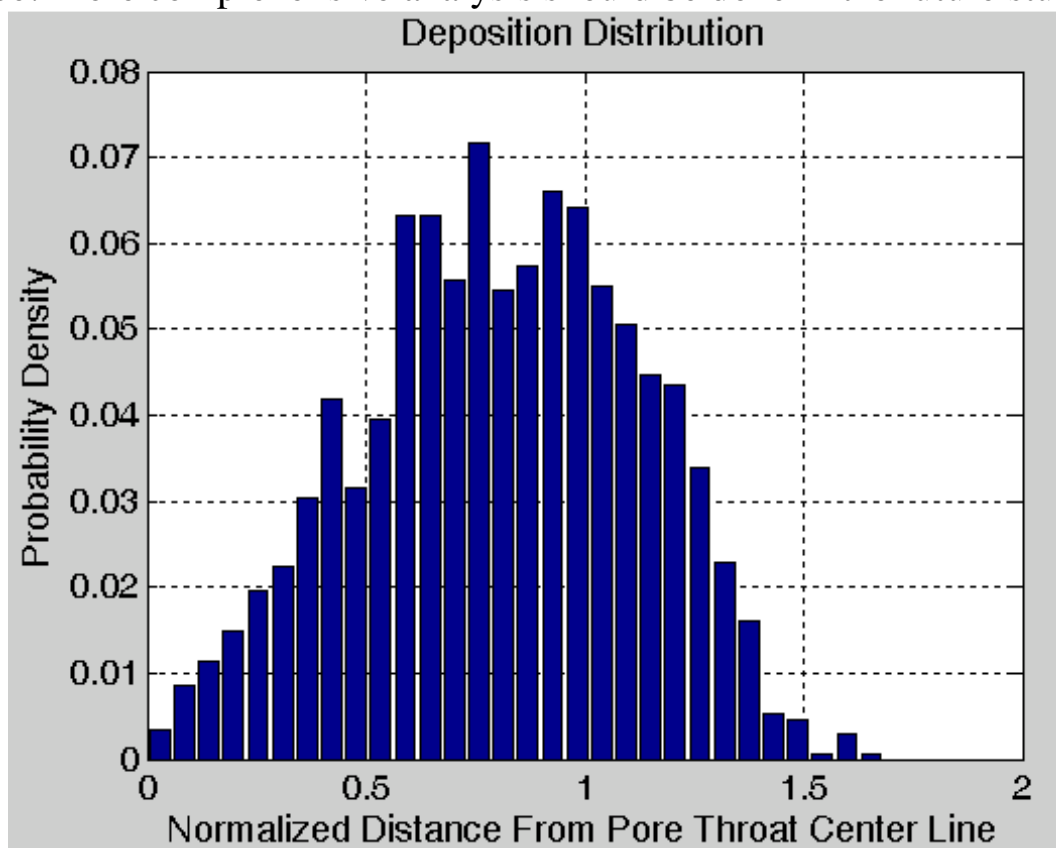

Figure 57. Deposition distibution of pore throats in Specimen 5 with porosity in the range of $40 \%-60 \%$.

Similar deposition distribution analysis is done for the pore throats with porosity higher than $60 \%$, and the analysis shows that more than $85 \%$ of the deposited particles are closer to the surface than to the center line of the pore throat. Moreover, a large portion of particle centers is outside the surface, which indicates that they are partially inside the pore throats. Clearly, for this porosity range, surface deposition model is most suitable to estimate the conductance of the throats.

Other sections of this specimen are also studied using similar methods, and the normalized permeability impairment is calculated and plotted in Figure 58. The permeability impairment is the highest at the entrance of the formation, with a $30 \%$ reduction. Deeper in the formation, more than $1 \mathrm{~mm}$ away from the formation face, the 
severity of permeability impairment reduces to about $5 \%$; and when it is more than $4 \mathrm{~mm}$ away from the formation face, the permeability reduction is much lower which can be ignored. Although the invasion depth is $8 \mathrm{~mm}$, the invaded particles do not reduce the permeability after $4 \mathrm{~mm}$, which is consistent with the porosity reduction situation. We can separate the specimen into three regions according to the damaged level: severe damaged region which is $0-1 \mathrm{~mm}$ away from the wellbore, intermediate damaged region which is 1 $4 \mathrm{~mm}$ away from the invasion start point, and intact region which is in the range of 4$8 \mathrm{~mm}$.

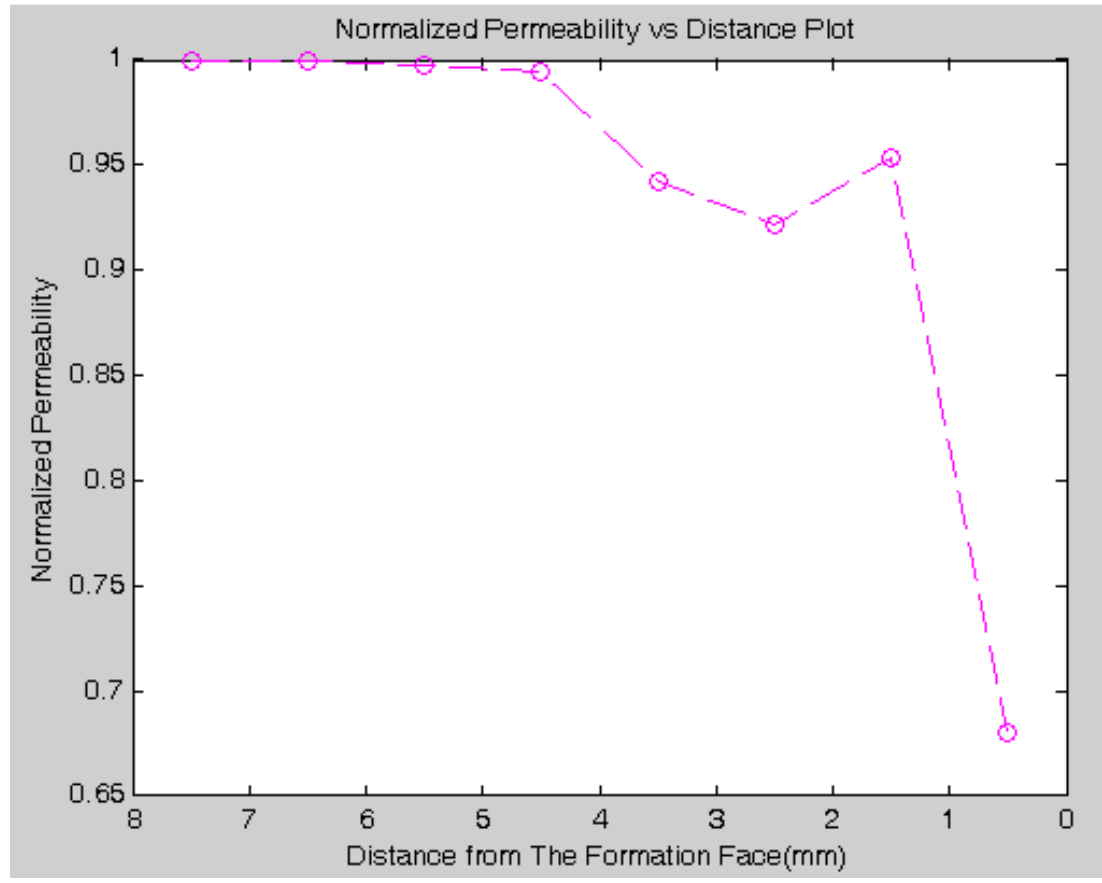

Figure 58. Normalized permeability along the distance from the formation face of Specimen 5.

\subsubsection{Permeability Impairment Study of Specimen 7}

Formation matrix in previous specimens is presented by equal-size sphere packs. To study the effect of the grain size sorting on formation damage, certain grain size range 
is selected according to the grain size distribution of Antler sandstone and feasibility of network permeability estimation.

Table 11. Properties of Specimen 7

\begin{tabular}{|c|c|}
\hline Properties & Value \\
\hline $\begin{array}{c}\text { Specimen Size } \\
\text { Length(mm)*Width(mm)*Height(mm) }\end{array}$ & $8 * 4 * 4$ \\
\hline Formation Grain Radius (mm) & $0.3-0.6$ \\
\hline Particle Size (mm) & $0.05-0.1$ \\
\hline Initial Porosity & $35 \%$ \\
\hline Damaged Porosity & $27 \%$ \\
\hline Invaded Depth (mm) & 8 \\
\hline
\end{tabular}

Similar as previous specimens, this specimen is divided into several sections based on the distance from the formation face, and the section which is closest to the invasion entrance is analyzed first.

Figure 59 shows the probability density plot of the pore throat size and the pore body size. The throat size distribution of this specimen is similar to Specimen 1, which has a lower initial porosity (30\%) and is packed from equal size grains; and is smaller than that of more porous Specimen 5. This indicates that poor sorting reduces the pore throat size. Note that about $2.5 \%$ of pore body size of this specimen is close to zero, which does not happen for equal sphere packing with a lower porosity. The cause of this phenomenon is the size difference of the spheres forming each pore and throat. 


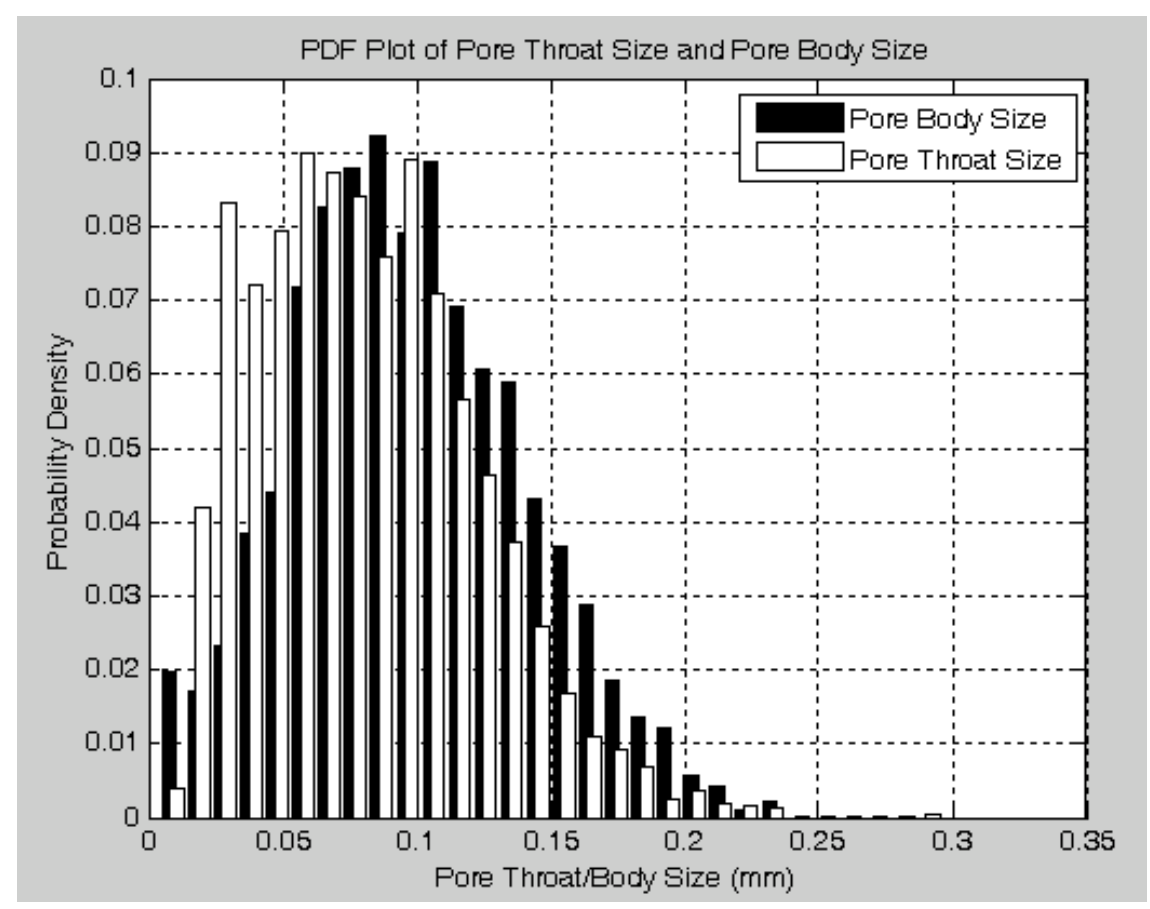

Figure 59. PDF plot of the pore throat size and the pore body size for Specimen 7.

The PDF plot of pore throat length (Figure 60) shows that pore throat length of Specimen 7 is slightly shorter than those of Specimen 1 and Specimen 5. 


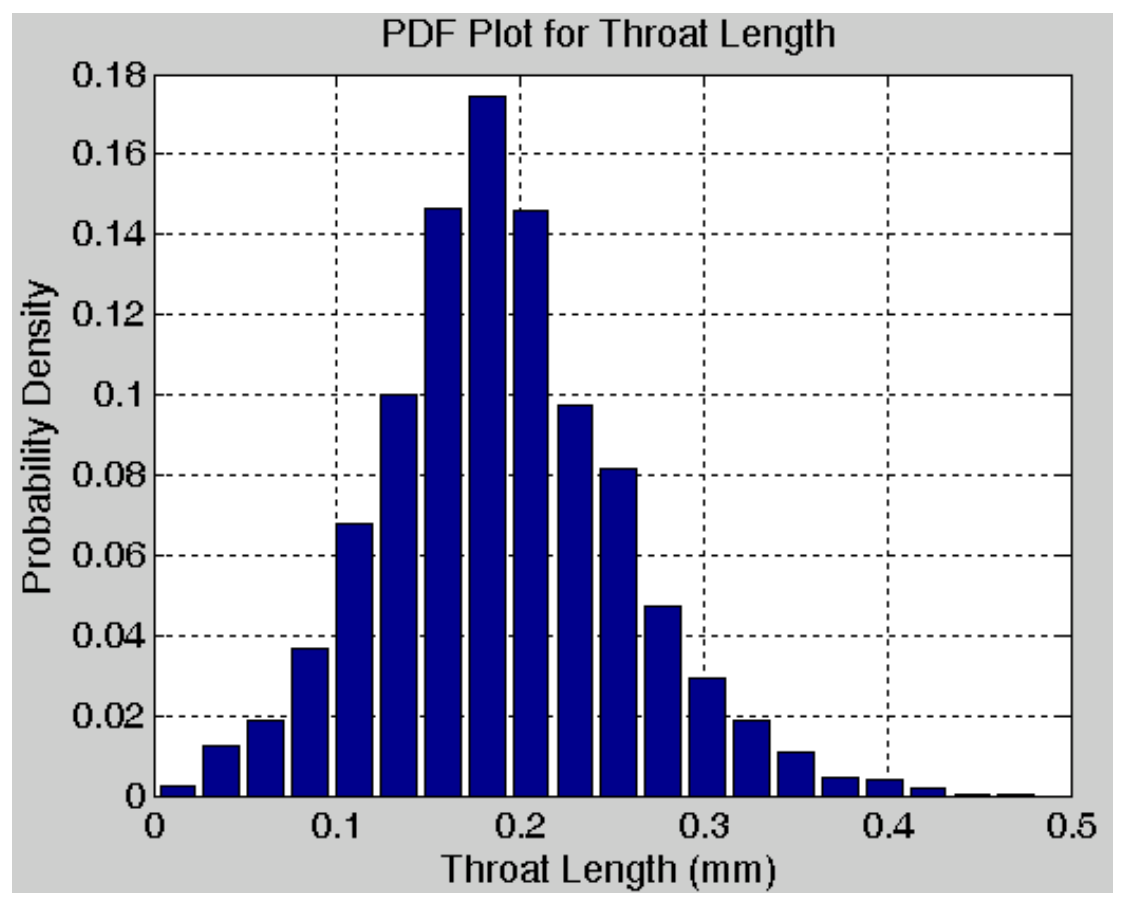

Figure 60. PDF plot of pore throat length for Specimen 7.

Figure 61 shows the histogram of the pore throats in the section closest to the formation face. Compared to Specimen 1 and Specimen 5, much more pore throats are found in this specimen, because of smaller sizes resulting more grains for the same volume. 


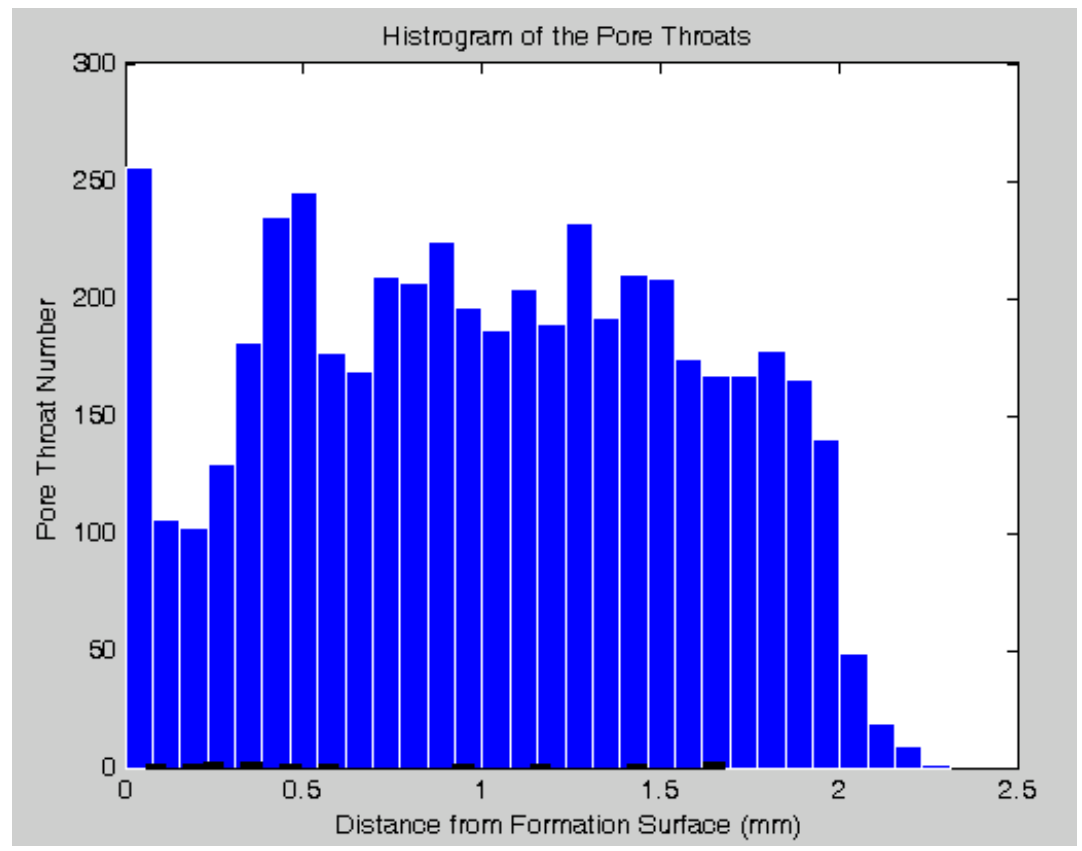

Figure 61. Histogram of all the pore throats and blocked pore throats along the distance from the formation face for Specimen 7.

From the PDF plot of the pore throat porosity shown in Figure 62, we can see that more than $50 \%$ pore throats contain penetrated particles. The percentage of invaded pore throats of this specimen is higher than Specimen 5, although the latter one has a higher initial porosity. This is very likely caused by the particle size difference between the two specimens, since finer particles can penetrated into more pores. However, in comparison to Specimen 5, most invaded pore throats still have relatively higher porosity. Therefore, permeability reduction of this specimen is expected to be lower than Specimen 5 . 


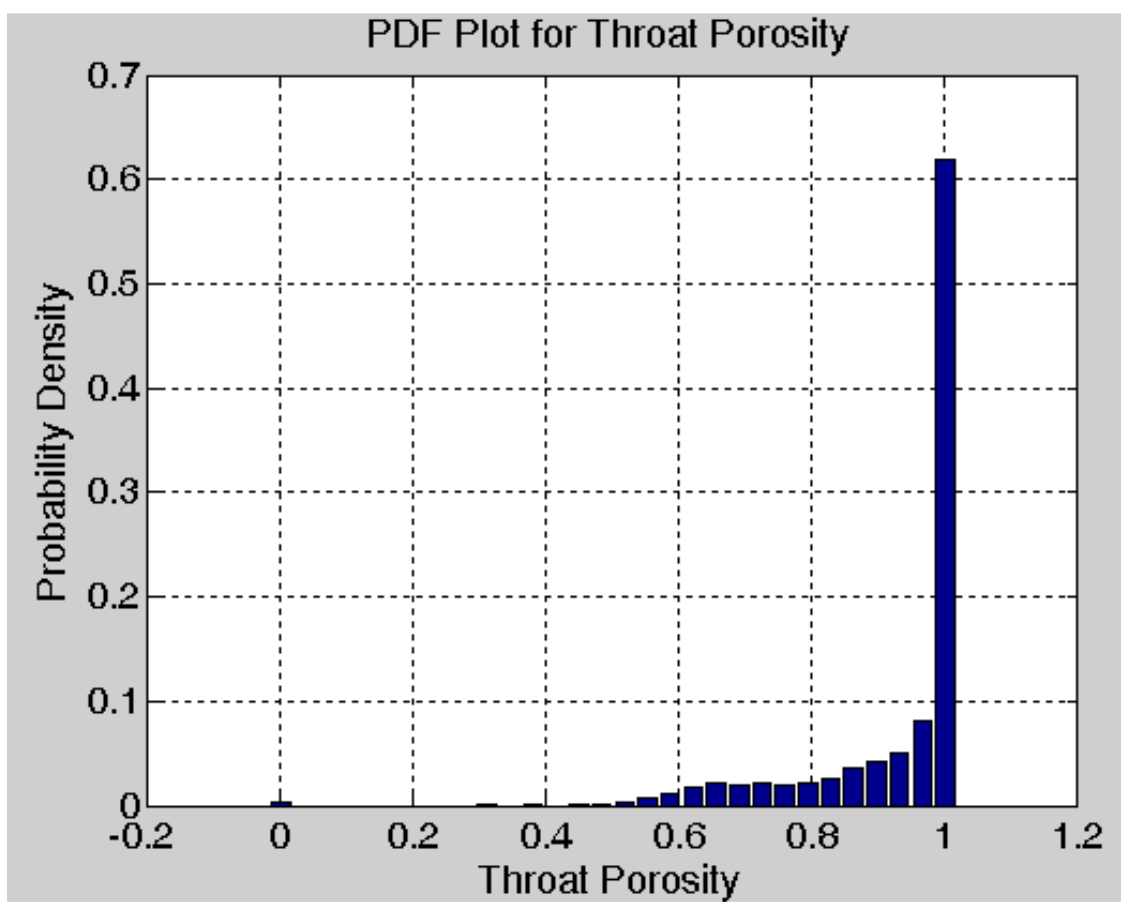

Figure 62. Histogram of all the pore throats and blocked pore throats along the distance from the formation face. The plot is generated from Specimen 7.

Figure 63 shows the probability density plot of the original pore volume vs the reduced pore volume. It is easy to see that pore volume of this specimen is smaller than Specimen 5, which is consistent with the pore body size comparison between the two specimens. For this specimen, around $70 \%$ of pore volume is smaller than $0.004 \mathrm{~mm}^{3}$ comparing to $50 \%$ of Specimen 5 pore volume. This difference is again caused by the relative smaller grain size in this specimen. Despite the absolute value, both specimens show the same trend of the pore volume reduction.

Figure 64 shows PDF plot of pore throat conductance, the conductance reduction of this specimen is less compared to Specimen 5. This can be caused by either a lower porosity of this specimen or more blocking on comparison to Specimen 5 . 


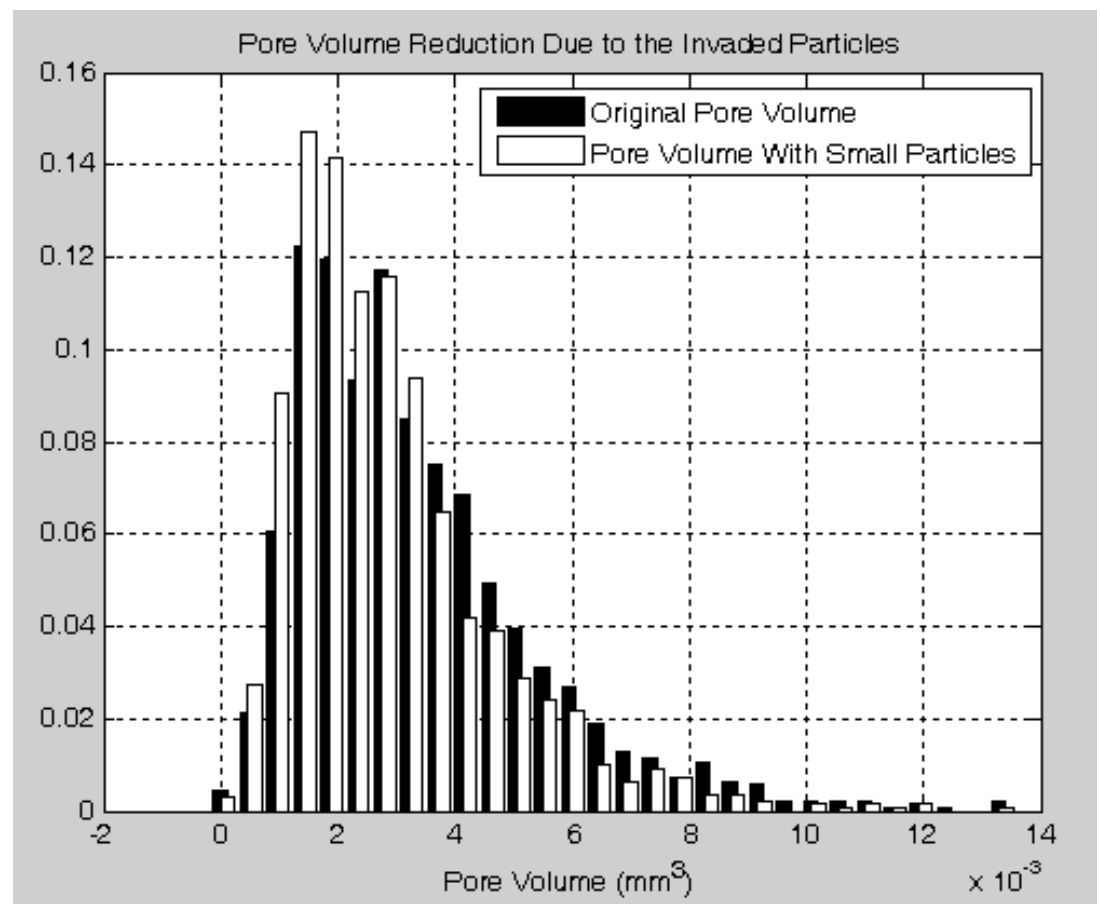

Figure 63. PDF plot of the original pore volume and the pore volume with small particles deposited in the pore bodies for Specimen 7.

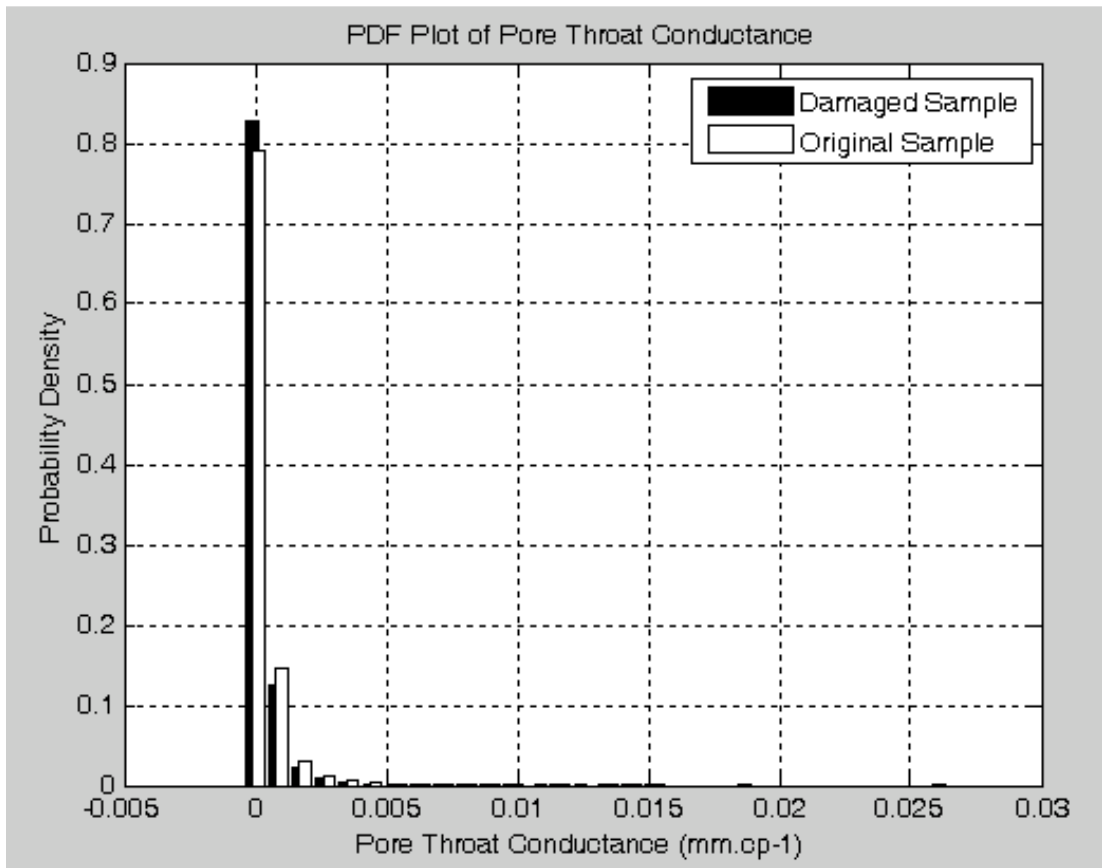

Figure 64. PDF plot of pore throat conductance for Specimen 7. 
Other sections of this specimen are also examined using the same method, and the estimated normalized permeability impairment is shown in Figure 65. The permeability reduction of the first section is about $25 \%$, which is lower than that of Specimen 5 , because smaller pore throat sizes result in less particles invasion. However, the permeability reduction of deeper sections of this specimen is higher than that of Specimen 1 and Specimen 5, which is due to finer particles penetrating deeper into the formation.

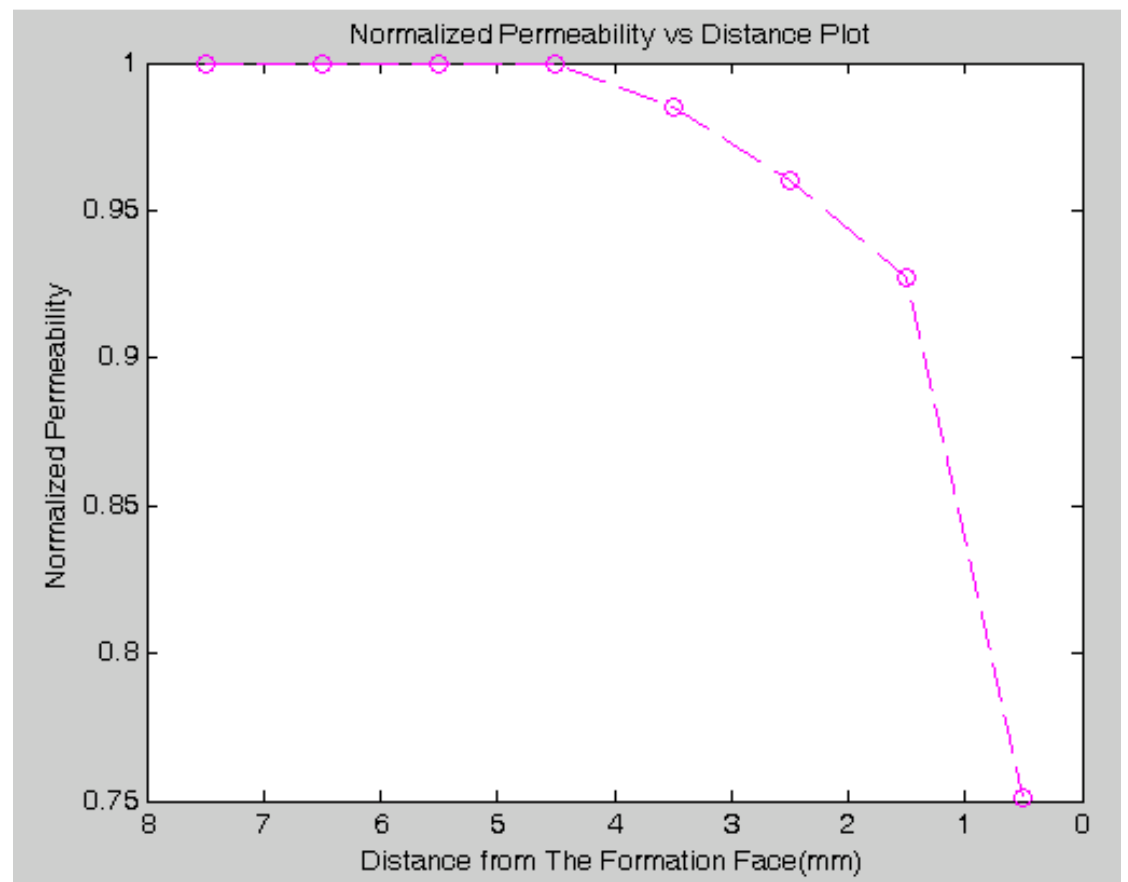

Figure 65. Normalized permeability along the distance from the formation face of Specimen 7. 


\section{Conclusions}

We quantified the severity of formation damage due to particle invasion based on various factors, among which are the initial porosity of formation, initial permeability of formation, external particle size distribution and fluid velocity.

\subsection{Effects of Particle Sizes on Formation Damage}

As shown in Figure 66, finer particles tend to reduce more porosity, since they can invade into more pores.

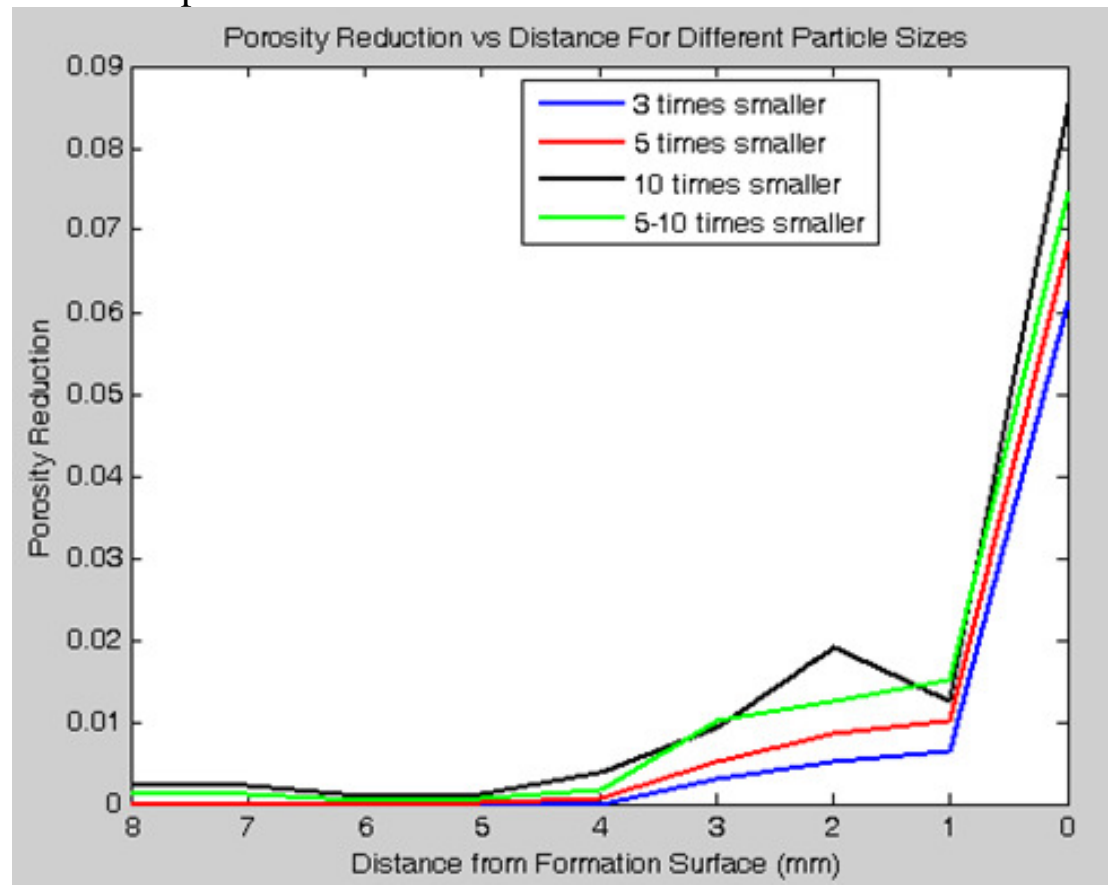

Figure 66. Porosity reduction along the distance from the formation face for different particle sizes. The formation specimen has initial porosity as $30 \%$.

However, when the particle sizes are small enough (in our case, more than five times smaller than the grain size), the porosity reduction of different particle sizes remains almost constant as shown in Figure 67. This finding is consistent with the previous analysis of the pore throat size distribution of the formation sample. From the study of the pore throat size distribution, around $70 \%$ pore throats are bigger than 
$0.05 \mathrm{~mm}$, which means particles, smaller than $0.05 \mathrm{~mm}$ (i.e. five times smaller than the grain size), can invade into most pores of the porous medium.

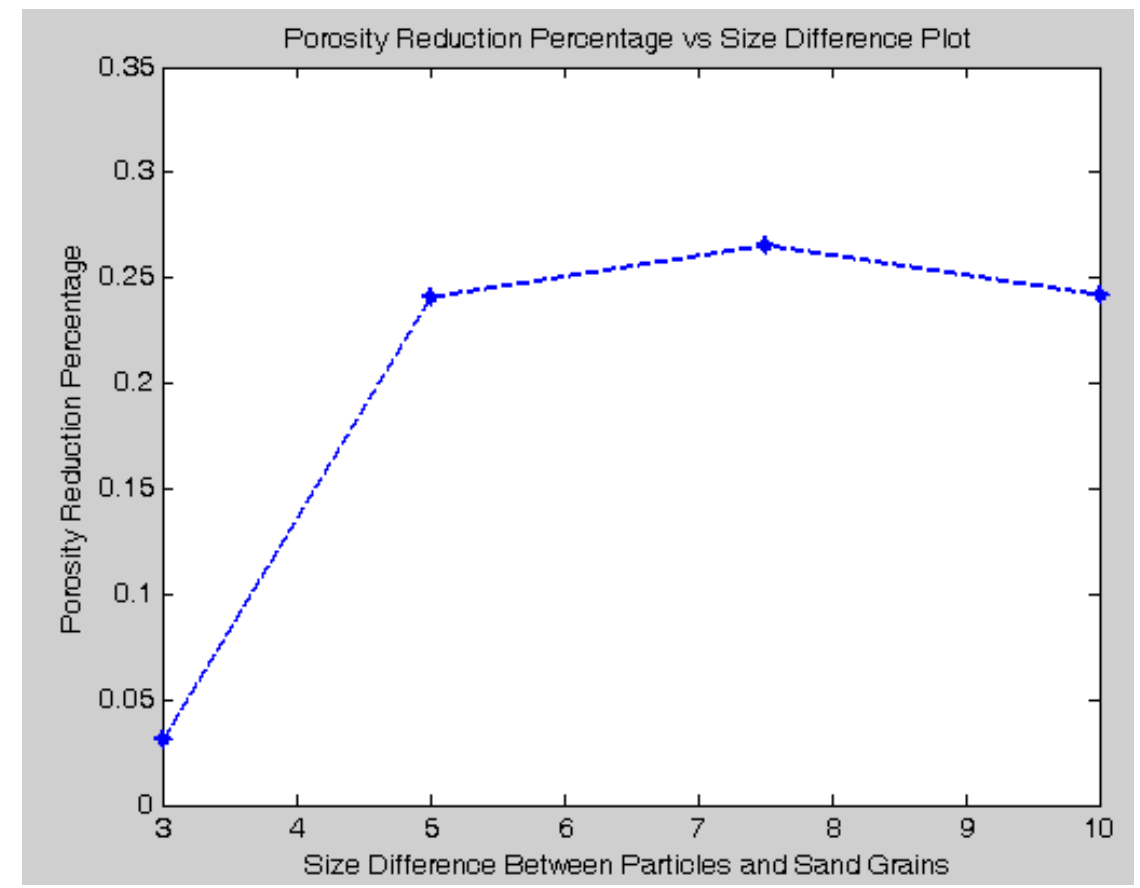

Figure 67. Total porosity reduction due to external particle invasion. The initial porosity of the specimen is 30\%. The particle sizes are 3 times smaller, 5 times smaller, 5-10 times smaller and 10 times smaller than formation grain size.

The particle fraction vs distance plot (see Figure 68) shows that bigger particles, which are three times smaller than formation grains, tend to clog at the formation face; while smaller particles, which are more than five times smaller than formation grains, penetrate deeper into the formation matrix. 


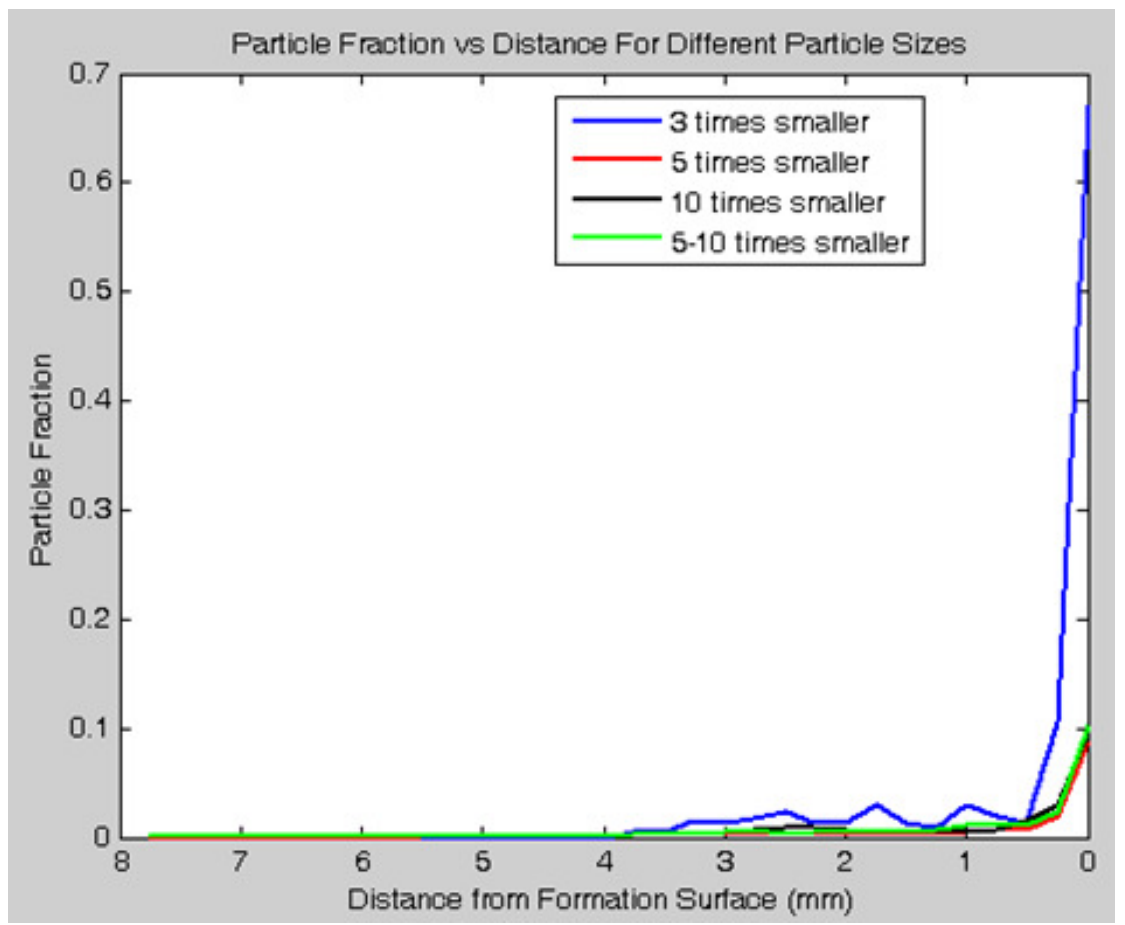

Figure 68. Invaded particle fraction along the distance from the formation face. The initial porosity of the specimen is $30 \%$; and the particle sizes are 3 times smaller, 5 times smaller, 5-10 times smaller and 10 times smaller than the formation grain size.

Figure 69 shows the permeability reduction (percentage) along the distance from the formation face. Invaded particles, which are ten times smaller than formation grains, reduce the original formation permeability by $35 \%$ at the near formation face section; while the big particles only reduce the original formation permeability by only $20 \%-25 \%$. 


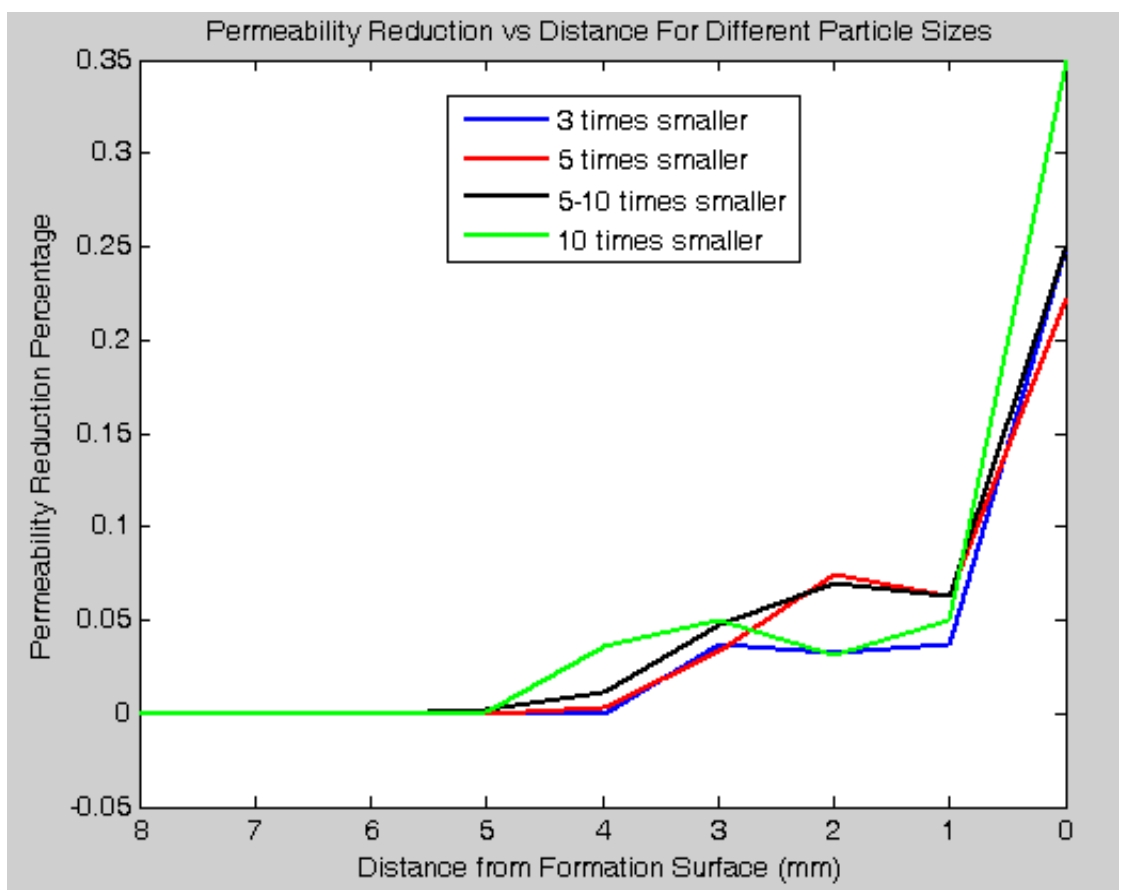

Figure 69. Permeability impairment due to particle invasion along the distance from the formation face. The initial porosity of the specimen is $30 \%$; and the particle sizes are 3 times smaller, 5 times smaller, 5-10 times smaller and 10 times smaller than the formation grain size.

In general, several conclusions can be drawn from the particle size study:

1. Particles of a smaller size penetrate deeper into the formation matrix, while bigger particles tend to clog at the formation surface.

2. Particles which are smaller than most pores reduce formation porosity more than those which are bigger than most pores.

3. Finer particles cause more permeability impairment at both the formation face and deep in the formation.

4. Compared to a uniform particle size distribution, a wide range of particle size distribution results in a more evenly distributed permeability impairment throughout the formation sample.

5. Permeability impairment due to particle invasion is dramatic at the near formation face area. For the smallest particle size used in this study, permeability impairment 
near formation face can reach about $35 \%$ of the original permeability for smallest particle size used in this study.

\subsection{EFFECT OF INITIAL Formation POROSITY ON FoRMATION DAMAGE}

As shown in Figure 70, the porosity reduction due to particle invasion is higher in a formation with a high initial porosity than in a formation with a low initial porosity. The figure also indicates that same size particles travel deeper in a high porosity formation than in a low porosity formation, since high porosity normally means bigger pore size in the porous medium for formation with equal size grains.

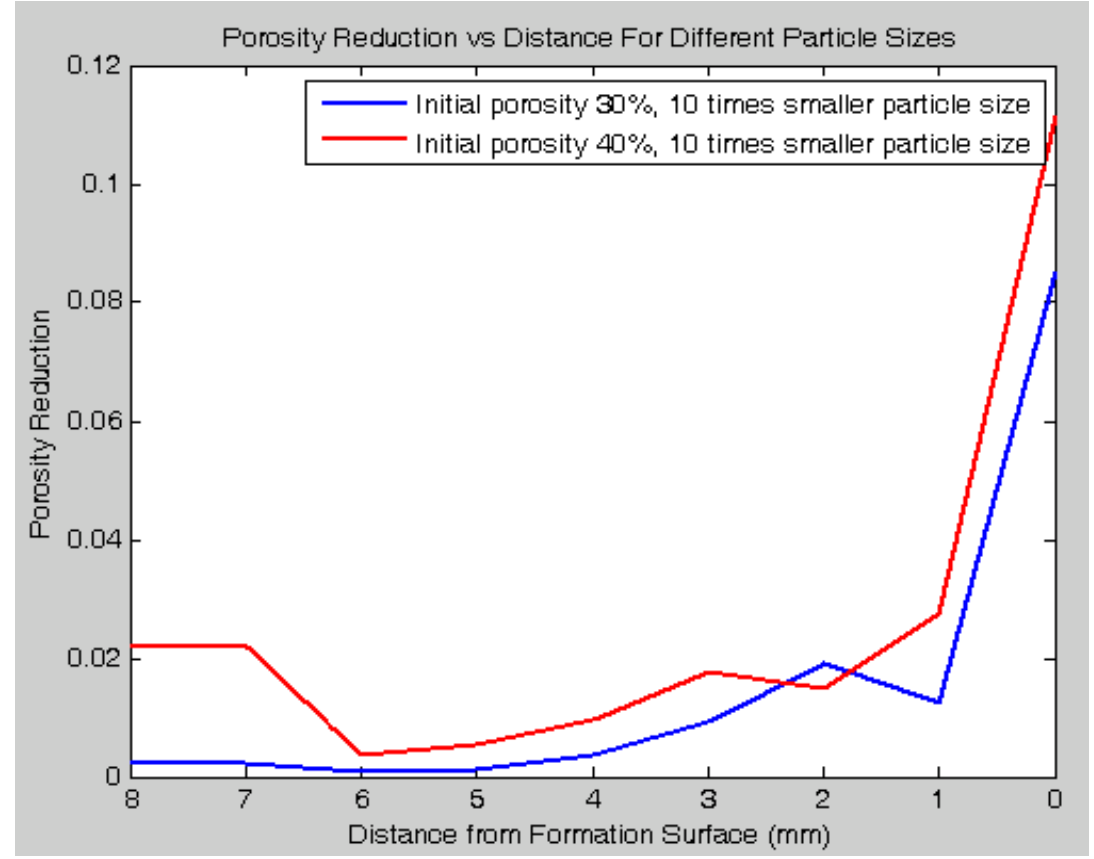

Figure 70. Porosity reduction vs. the distance from the formation face for different initial formation porosities. The invaded particle size is ten times smaller than that of the formation grains.

The particle fraction vs distance plot (see Figure 71) shows that formation samples with two different initial porosities have similar invaded particle distributions along the distance. This is because the particle size is small enough to penetrate most of the pore throats even in low porosity formation. 


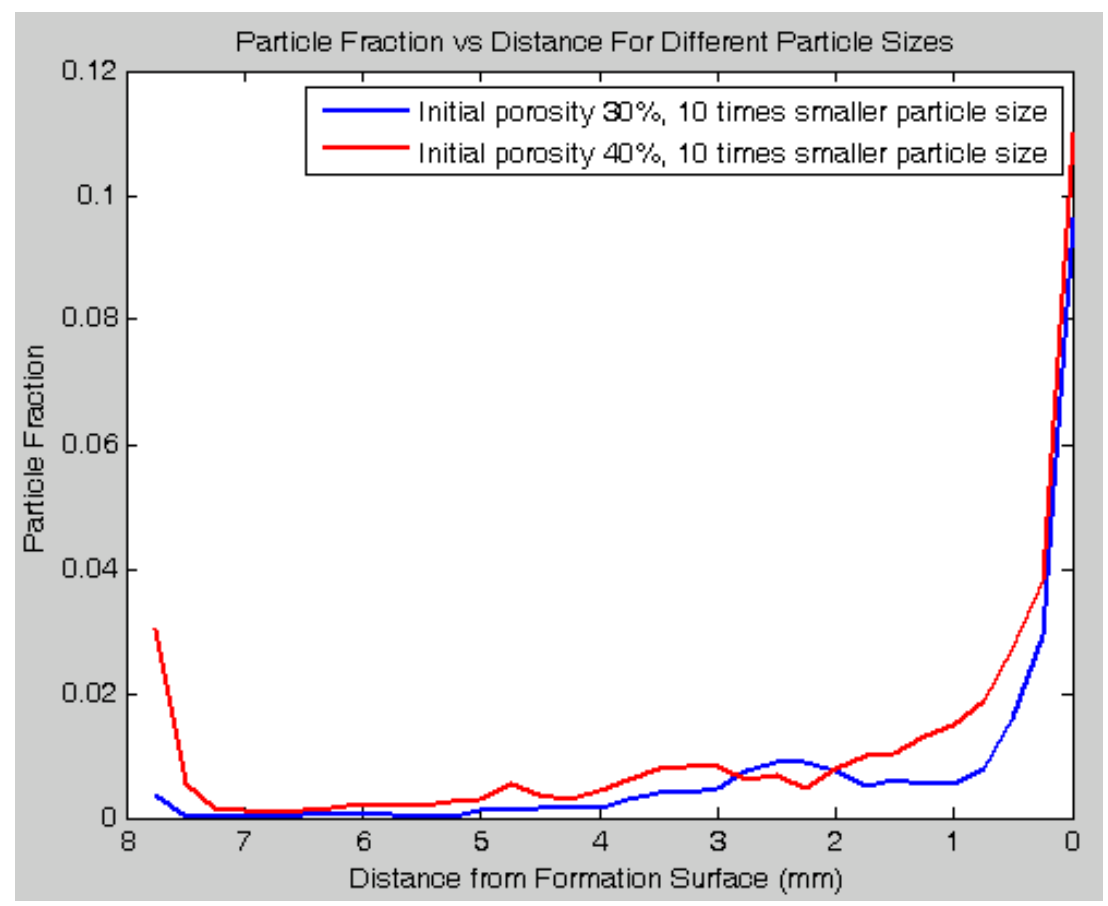

Figure 71. Invaded particle fraction along the distance from the formation face. The particle size is ten times smaller than formation grains; and the initial formation porosity is $30 \%$ and $40 \%$.

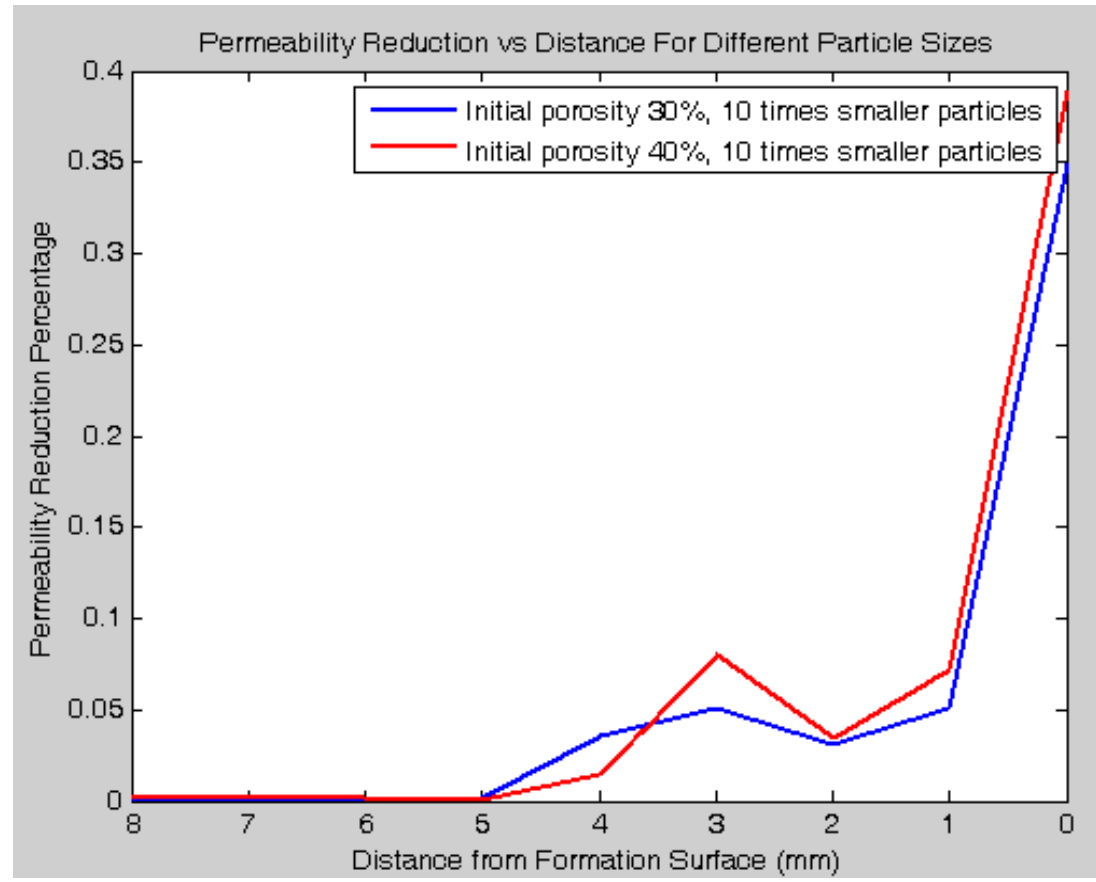

Figure 72. Permeability impairment due to particle invasion along the distance from the formation face. The particle size is ten times smaller than formation grains; and the initial formation porosity is $30 \%$ and $40 \%$. 
Similar to porosity reduction due to particle invasion, permeability impairment of a high porosity formation is also higher than that of a low porosity formation. At the formation face, the permeability impairment of a high porosity formation reaches almost $40 \%$, which is a dramatic drop in formation permeability. Remedial action should be taken to maintain the productivity of the well.

Overall, a higher initial formation porosity causes more severe formation damage. Therefore, particle size distributions should be carefully designed according to the structure of formations to reduce formation damage.

\subsection{Model APPlications}

The particle invasion simulation and permeability estimation model proposed in this study can serve as a tool to predict the formation damage according to the given parameters, such as the formation porosity, the formation grain size, the particle size distribution, the fluid viscosity, the differential pressure between a wellbore and a formation and the circulation velocity. This model can also help design the drilling fluid solids according to the given formation properties, and thus control the formation damage. Furthermore, the approach to estimating poorly-sorted formation permeability can be applied to estimate the permeability of other kinds of damaged formations, such as formations containing crushed sands.

\subsection{MODEL LimitationS}

There are also some limitations of the proposed model:

1. Due to current model capability of $\mathrm{PFC}^{3 \mathrm{D}}$, the fluid flow is simplified for the particle invasion simulation. We assumed a constant fluid velocity, which is not realistic. Moreover, the particle-fluid interaction is also simplified.

2. The permeability estimation model works better for the formation with equal 
size grains. Although the particle invasion simulation works well for a wide grain size distribution, permeability estimation is less reliable for a poorly sorted formation.

3. For the simulation, only the mechanical interaction between particles and formation grains is taken into account; however, there are various other forces interacting between the particles and the formation grain face. 


\section{Future Work}

This thesis aims to simulate particle invasion and to estimate permeability of damaged formations. The platform for such a model is built in this study, and various effects on formation damage are studied. Further studies, which are listed below, could be done based on the model.

1. Study the external mud cake build up time and permeability based on given particle size distributions, circulation velocities and fluid properties.

Mud cake build up time and permeability is vital for controlling loss of circulation. Proper design of these factors can shorten the mud cake build up time and reduce the mud cake permeability.

2. Verify the simulation results with experimental data.

The particle simulation model proposed in this thesis is based mainly on the mechanical interaction between particles and formation grains and the hydrodynamic drag forces exerted by invading fluids on the particles. Other forces between particles and the formation grain surface are not considered in the model. Experimental data can be used to validate and calibrate the model by adding the forces ignored.

3. Study particle invasion in fractured formations.

The discrete element method can be used to simulate formation fracturing. Therefore, a more comprehensive model can be built to simulate formation fracture followed by a simulation of particles invasion to fractured formation. The permeability estimation model built in this study can be used to estimate the resultant permeability.

4. Study particle invasion for different well trajectories. 
The simulation for particle invasion proposed in this study is for vertical wells; however, by simply changing the applied force on the particles, particle invasion into horizontal and deviated wells can be simulated.

5. Predict the mobility of deposited particles during cleanup.

Drilling induced formation damage can be controlled and reduced, but cannot be completely avoided. Therefore, effective formation cleanup plays an important role in well productivity. The model used in this study can also be applied to the cleanup procedure after proper adjustment. To simulate cleanup, hydrodynamic forces can be applied in the opposite direction and the movement of the deposited particles under the hydrodynamic forces can be simulated by $\mathrm{PFC}^{3 \mathrm{D}}$. The permeability estimation approach proposed in this study can be used to estimate the permeability of the formation after cleanup.

6. Predict water saturation change and relative permeability change due to formation damage.

Due to differential pressure between a wellbore and a formation, drilling fluids are forced into the formation near wellbore and replace oil there. There is still some residual oil within the drilling fluid invaded formation.

7. Study the formation damage in shale.

Formation damage in shale is still puzzling. The void space of intact shale is mostly disconnected nano-pores. However, shale is very brittle and the drilling process tends to induce a network of micro-fractures, without completely disintegrating the rock due to compressive stresses. The question of formation damage then is the plugging and bridging of the micro-fracture network, and the resulting permeability impairment has not been studied to date. 
8. PFC software is very well set up for estimating seismic velocities based the graingrain contact network. Since most well logging measurements are done near wellbore (where damage is the largest), it would be interesting to investigate the influence of invaded particles (formation damage) on primary and secondary seismic wave velocities. 


\section{APPENDIX}

\section{A. Generate a Dense Sphere Pack in PFC ${ }^{3 D}$}

To generate a dense spherical packing in $\mathrm{PFC}^{3 \mathrm{D}}$, initially, a smaller grain size is used to generate the particle assembly (see Figure 73), and then the grain size is increased to the target size which results in the overlapping between particles. Consequently, overlapping causes high internal forces within the generated packing. Computation cycles are performed to release the internal forces by allowing the movement of the particles, as shown in Figure 74. Black arrows in the figure represent the velocities of the particles. Relative equilibrium status is reached when the velocities of the particles are very low.

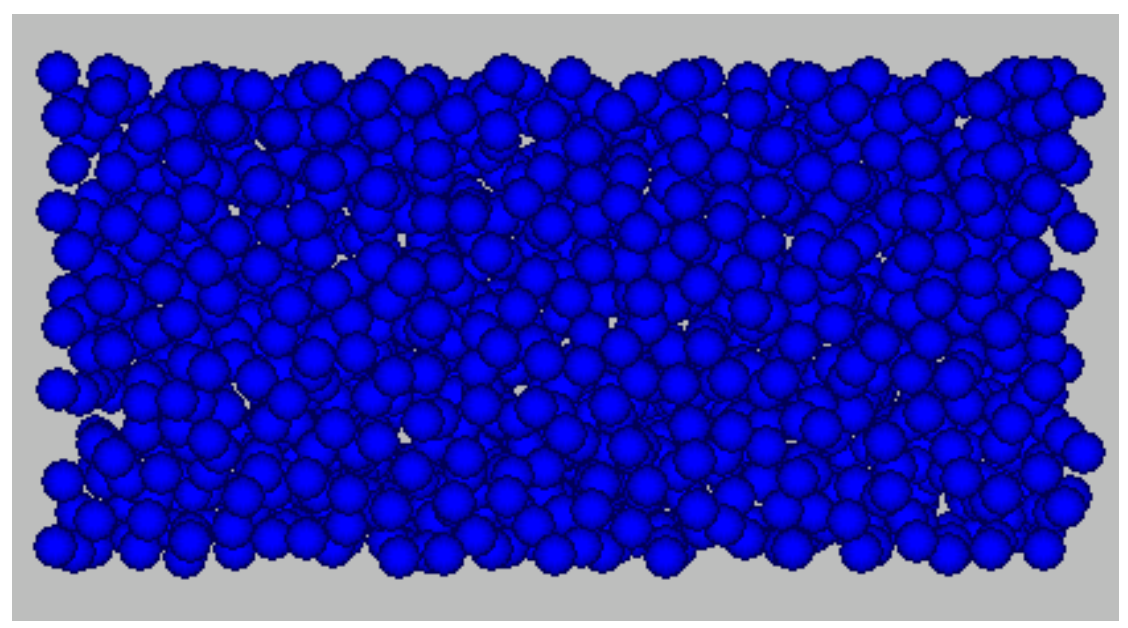

Figure 73. A sphere pack with a smaller grain size is generated first 


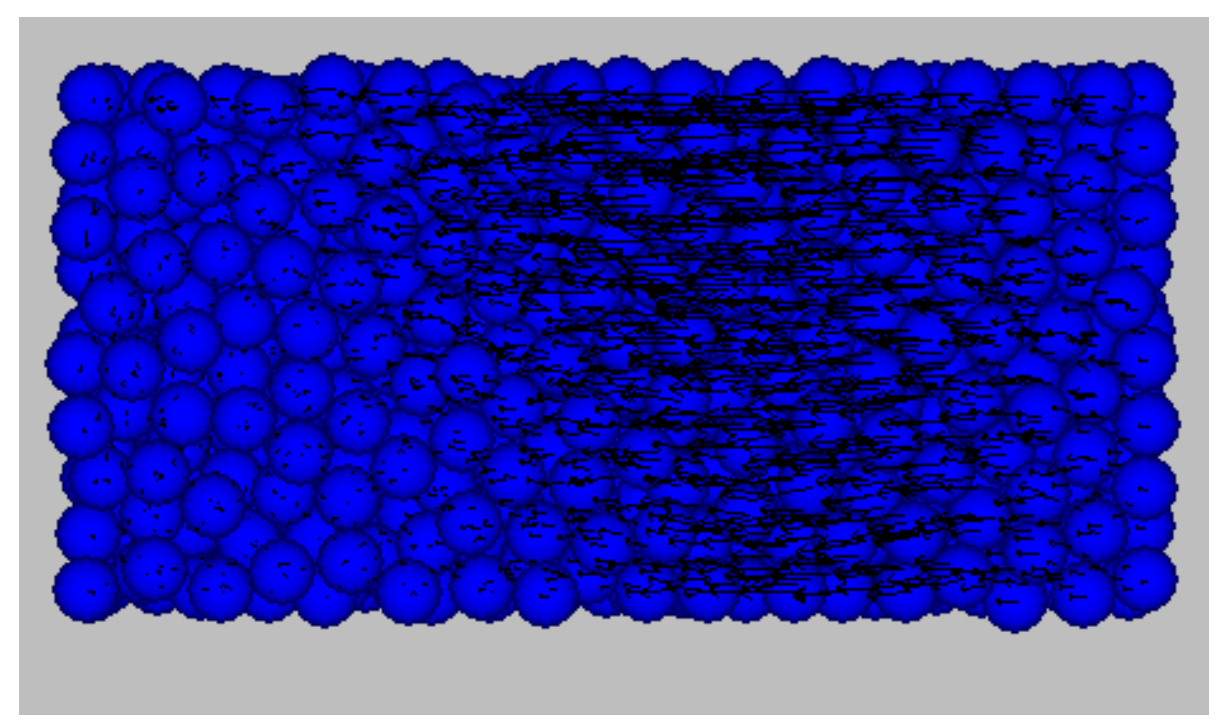

Figure 74 . The grain size of the sphere pack is increased to the target size.

Computation cycle is performed to relase high internal forces caused by overlapping between particles. 


\section{B. Throat Conductance Calculation}

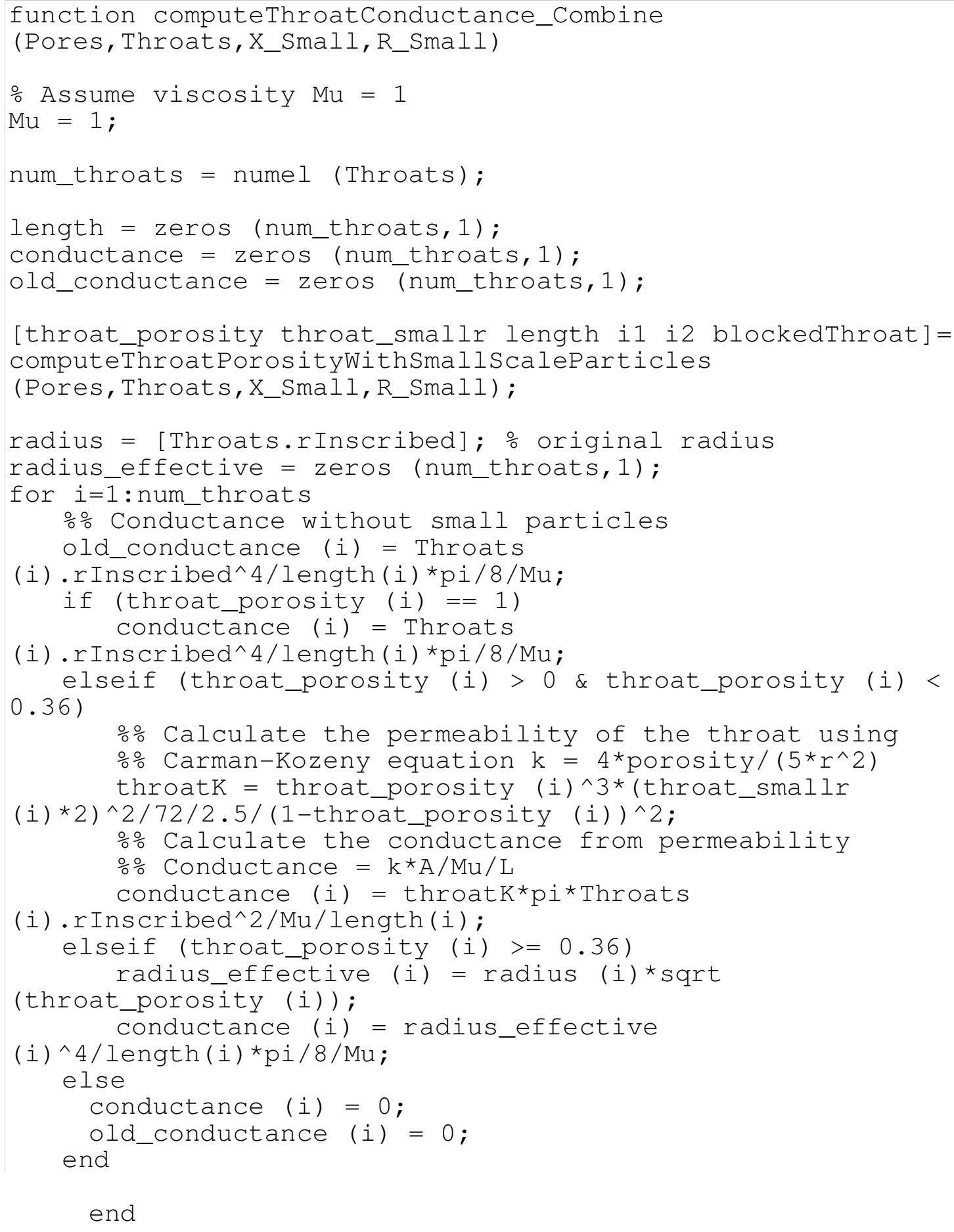




\section{Throat Porosity Calculation}

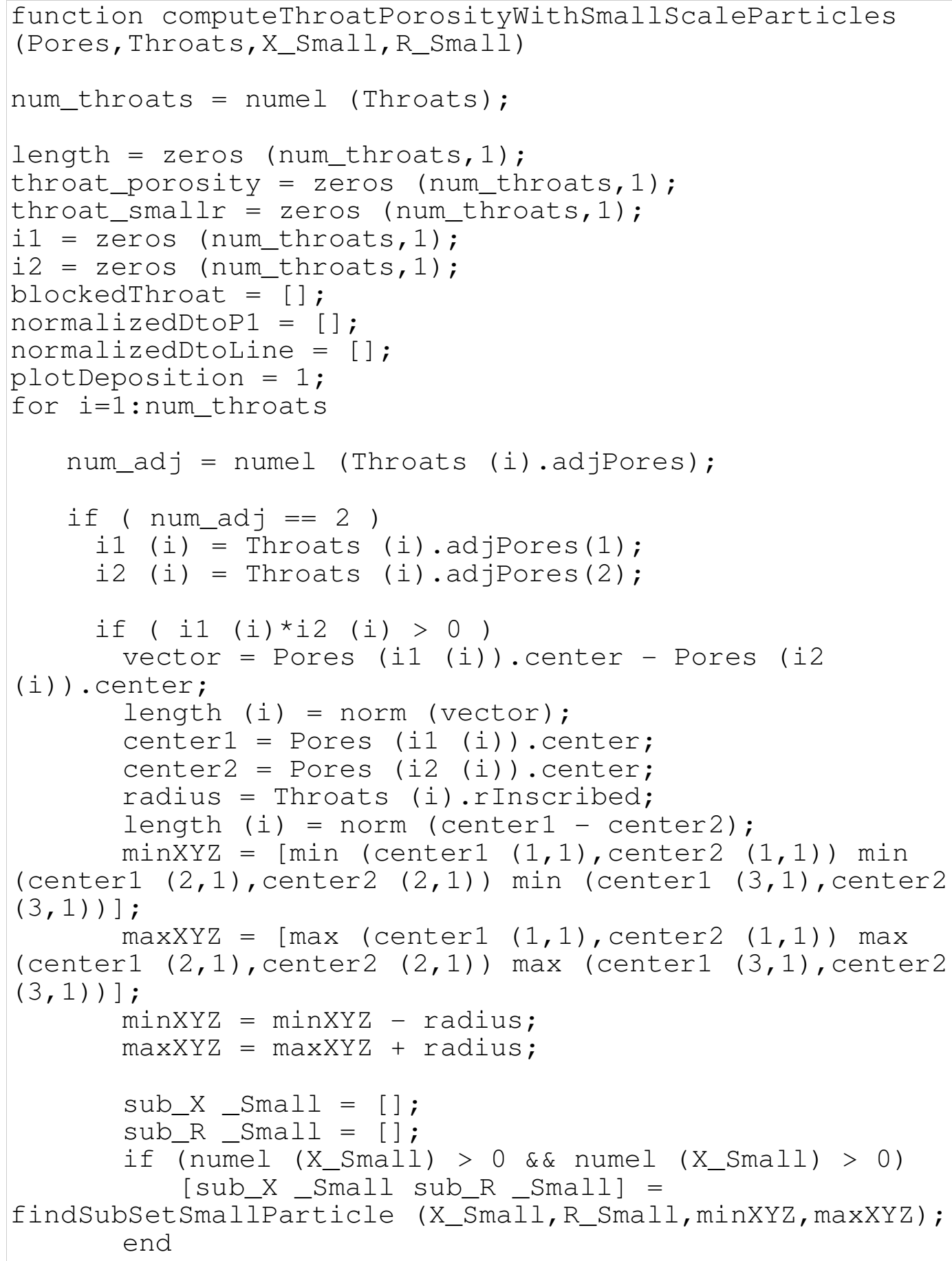




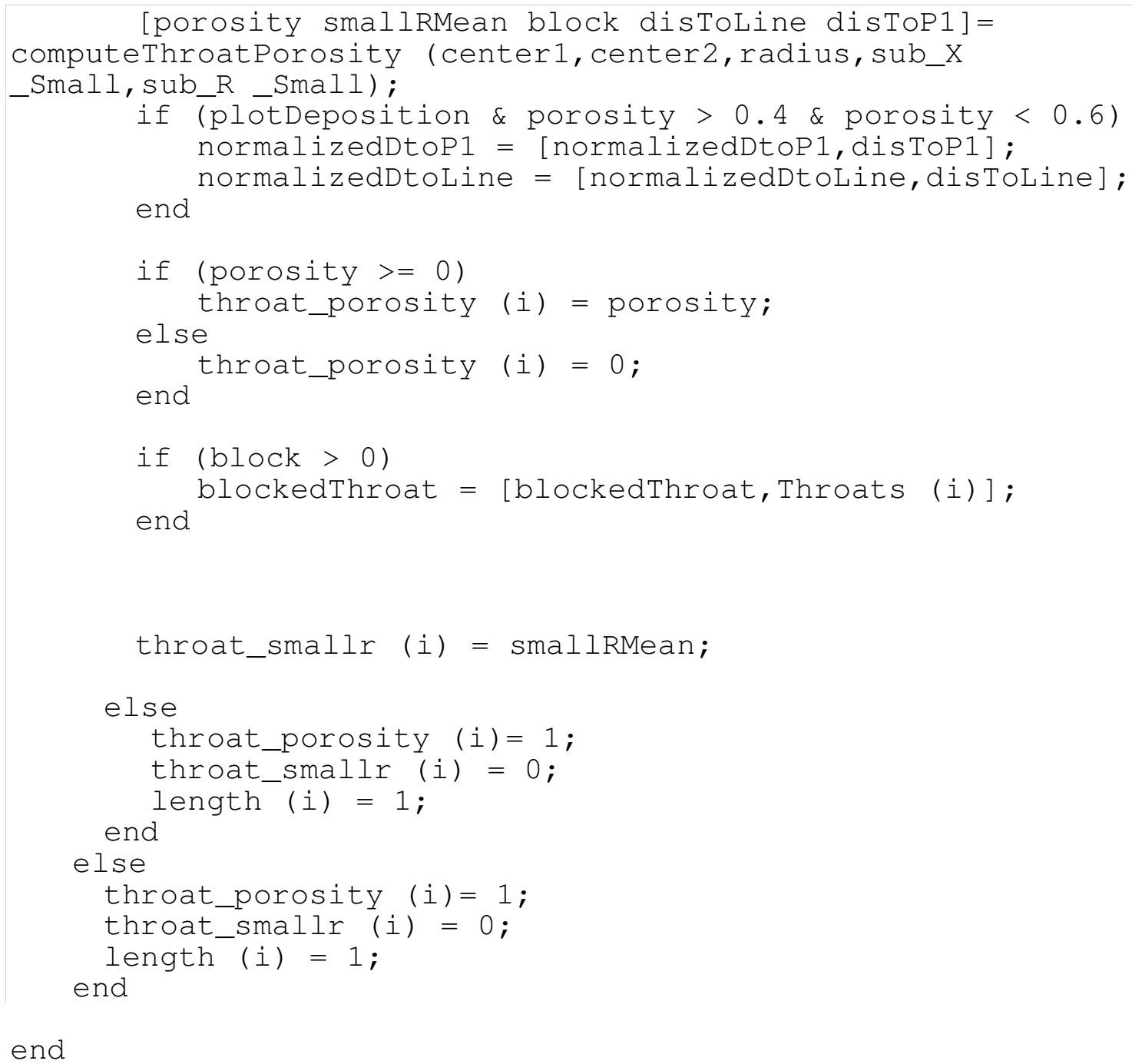




\section{References}

[1] A. K. Wojtanowicz, Z. Krilov, and J. P. Langlinais, "Study on the Effect of Pore Blocking Mechanisms on Formation Damage," presented at the SPE Production Operations Symposium, 1987.

[2] K. C. Khilar, and H. S. Fogler," "Colloidally Induced Fines Migration in Porous Media," Reviews in Chemical Engineering, vol. 4, no. 1-2, pp. 41-108, Jun. 1987.

[3] C. Gruesbeck and R. E. Collins, "Entrainment and Deposition of Fine Particles in Porous Media," Society of Petroleum Engineers Journal, vol. 22, no. 6, Dec. 1982. [4] M. M. Sharma and Y. C. Yortsos, "Fines migration in porous media," AIChE Journal, vol. 33, no. 10, pp. 1654-1662, 1987.

[5] S. D. Rege and H. S. Fogler, “A network model for deep bed filtration of solid particles and emulsion drops," AIChE Journal, vol. 34, no. 11, pp. 1761-1772, Nov. 1988.

[6] S. Bortal-Nafaa and D. Gouvenot, Fine cement grout injection: discrete numerical modeling, Numerical modeling in micromechanics via particle methods: proceedings of the 1st International PFC Symposium. Gelsenkirchen, Germany: Taylor \& Francis, 2002.

[7] Y. S. Kim and A. J. Whittle, "Filtration in a Porous Granular Medium: 1. Simulation of Pore-Scale Particle Deposition and Clogging," Transport in Porous Media, vol. 65 , no. 1 , pp. $53-87$, Oct. 2006.

[8] L. Li and R. M. Holt, "Particle Scale Reservoir Mechanics," Oil \& Gas Science and Technology, vol. 57, pp. 525-538, 2002.

[9] E. Oort, J. F. G. Velzen, and K. Leerlooijer, "Impairment by Suspended Solids Invasion: Testing and Prediction," SPE Production \& Facilities, vol. 8, no. 3, Aug. 1993. 
[10] F. Al-Abduwani, A. Shirzadi, W. van den Brock, and P. Currie, "Formation Damage vs. Solid Particles Deposition Profile During Laboratory-Simulated ProducedWater Reinjection,” SPE Journal, vol. 10, no. 2, Jun. 2005.

[11] D. Wildenschild, C. M. P. Vaz, M. L. Rivers, D. Rikard, and B. S. B.

Christensen, "Using X-ray computed tomography in hydrology: systems, resolutions, and limitations," Journal of Hydrology, vol. 267, no. 3-4, pp. 285-297, Oct. 2002.

[12] R. E. Gilliland and M. E. Coles, "Use of CT Scanning in the Investigation of Damage to Unconsolidated Cores," presented at the SPE Formation Damage Control Symposium, 1990.

[13] P. A. Cundall and O. D. L. Strack, "A discrete numerical model for granular assemblies," Géotechnique, vol. 29, no. 1, pp. 47-65, Jan. 1979.

[14] Itasca Consulting Group, Inc., "PFC3D 3.1 User’s Guide.” 2005.

[15] Itasca Consulting Group, Inc., "PFC3D 3.1 Theory and Background.” 2005.

[16] R. D. Mindlin and H. Deresiewicz, "Elastic spheres in contact under varying oblique forces," Journal of Applied Mechanics, vol. 20, pp. 327-344, 1953.

[17] H. Wang, G. R. Krishnan, J.-C. Roegiers, and M. Zaman, "Behavior of natural and remolded Antler Sandstone: Sample preparation and grain crushing aspects," presented at the The 35th Symposium on Rock Mechanics (USRMS), Reno, NV, 1995. [18] I. GIL, J. C. Roegiers, R. Hart, and Y. Shimizu, "Modeling the Mechanical Properties of Antler Sandstone Using a Discrete Element Model," American Rock Mechanics Association.

[19] A. Suri and M. Sharma, "Strategies for Sizing Particles in Drilling and Completion Fluid.," SPE Journal, vol. 9, no. 1, Mar. 2004.

[20] N. H. Mondol, J. Jahren, K. Bjollykke, and I. Brevik, "Elastic properties of clay minerals," The Leading Edge, vol. 27, no. 6, p. 758, 2008. 
[21] I. Fatt, “The Network Model of Porous Media," Petroleum Transactions, AIME, vol. 207, pp. 144-181, 1956.

[22] M. J. Blunt, "Flow in porous media -- pore-network models and multiphase flow," Current Opinion in Colloid \& Interface Science, vol. 6, no. 3, pp. 197-207, Jun. 2001.

[23] G. Mason and N. R. Morrow, "Capillary behaviour of a perfectly wetting liquid in irregular triangular tubes," J. Colloid Interface Sci., vol. 141, p. 262-274, 1991.

[24] S. L. Bryant, G. Mason, and D. Mellor, "Quantification of Spatial Correlation in Porous Media and Its Effect on Mercury Porosimetry," J. Colloid Interface Sci., vol. 177, p. $88-100,1996$.

[25] R. I. Al-Raoush, K. Thompson, and C. W. Willson, "Comparison of Network Generation Techniques for Unconsolidated Porous Media," Soil. Sci. Soc. Am. J., vol. 67, p. 1687-1700, 2003.

[26] M. de Berg, M. van Kreveld, M. Overmars, and O. Schwarzkopf, Computational Geometry. New York: Springer Verlag, 2000.

[27] P.-E. Øren and S. Bakke, "Reconstruction of Berea sandstone and pore-scale modelling of wettability effects," Journal of Petroleum Science and Engineering, vol. 39, no. 3-4, pp. 177-199, Sep. 2003.

[28] M. Prodanovic, W. B. Lindquist, and R. S. Seright, "Porous structure and fluid partitioning in polyethylene cores from 3D X-ray microtomographic imaging," Journal of Colloid and Interface Science, vol. 298, no. 1, pp. 282-297, Jun. 2006.

[29] J. Behseresht, S. Bryant, and K. Sepehrnoori, "Infinite-Acting Physically Representative Networks for Capillarity-Controlled Displacements," presented at the SPE Annual Technical Conference and Exhibition, 2007. 
[30] G. Mason and D. Mellor, "Simulation of drainage and imbibition in a random packing of equal spheres," J. Colloid Interface Sci., vol. 176, no. 1, p. 214-225, 1995.

[31] T. Patzek, "Verification of a complete pore network simulator of drainage and imbibition,” 2000.

[32] P. H. Valvatne and M. J. Blunt, "Predictive pore-scale modeling of two-phase flow in mixed wet media," Water Resour. Res., vol. 40, p. W07406, 2004.

[33] F. A. L. Dullien, Porous Media, Second Edition: Fluid Transport and Pore Structure, 2nd ed. Academic Press, 1991. 